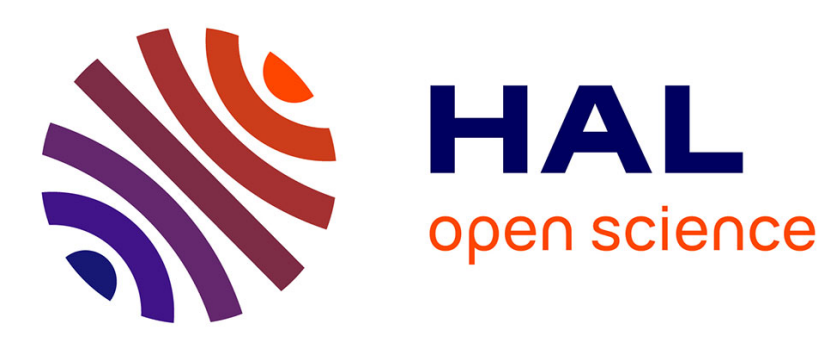

\title{
Large sample properties of the matrix exponential spatial specification with an application to FDI
}

Nicolas Debarsy, Fei Jin, Lung-Fei Lee

\section{To cite this version:}

Nicolas Debarsy, Fei Jin, Lung-Fei Lee. Large sample properties of the matrix exponential spatial specification with an application to FDI. 2013. hal-00858174v1

\section{HAL Id: hal-00858174 \\ https://hal.science/hal-00858174v1}

Preprint submitted on 4 Sep 2013 (v1), last revised 7 Oct 2014 (v3)

HAL is a multi-disciplinary open access archive for the deposit and dissemination of scientific research documents, whether they are published or not. The documents may come from teaching and research institutions in France or abroad, or from public or private research centers.
L'archive ouverte pluridisciplinaire HAL, est destinée au dépôt et à la diffusion de documents scientifiques de niveau recherche, publiés ou non, émanant des établissements d'enseignement et de recherche français ou étrangers, des laboratoires publics ou privés. 


\title{
Large sample properties of the matrix exponential spatial specification with an application to FDI
}

\author{
Nicolas Debarsy ${ }^{\mathrm{a}, 1}$, Fei Jin ${ }^{\mathrm{b}}$, Lung-fei Lee ${ }^{\mathrm{c}}$ \\ ${ }^{a}$ Univ. Orléans, CNRS, LEO, F45067, Orléans, France \\ and CERPE, University of Namur, Rempart de la Vierge, 8, B-5000 Namur \\ ${ }^{b}$ School of Economics, Shanghai University of Finance and Economics, Shanghai 200433, China \\ ${ }^{c}$ Department of Economics, The Ohio State University, Columbus, OH 43210, USA
}

\begin{abstract}
This paper considers the large sample properties of the matrix exponential spatial specification (MESS) and compares its properties with those of the spatial autoregressive (SAR) model. We find that the quasimaximum likelihood estimator (QMLE) for the MESS is consistent under heteroskedasticity, a property not shared by the QMLE of the SAR model. For the MESS in both homoskedastic and heteroskedastic cases, consistency is proved and asymptotic distributions are derived. We also consider properties of the generalized method of moments estimator (GMME). In the homoskedastic case, we derive a best GMME that is as efficient as the maximum likelihood estimator under normality and can be asymptotically more efficient than the QMLE under non-normality. In the heteroskedastic case, an optimal GMME can be more efficient than the QMLE asymptotically and the possible best GMME is also discussed. For the general model that has MESS in both the dependent variable and disturbances, labeled MESS(1,1), the QMLE can be consistent under unknown heteroskedasticity when the spatial weights matrices in the two MESS processes are commutative. Also, properties of the QMLE and GMME for the general model are considered. The QML approach for the MESS model has the computational advantage over that of a SAR model. The computational simplicity carries over to MESS models with any finite order of spatial matrices. No parameter range needs to be imposed in order for the model to be stable. Furthermore, the Delta method is used to derive test statistics for the impacts of exogenous variables on the dependent variable. Results of Monte Carlo experiments for finite sample properties of the estimators are reported. Finally, the MESS(1,1) is applied to Belgium's outward FDI data and we observe that the dominant motivation of Belgium's outward FDI lies in finding cheaper factor inputs.
\end{abstract}

Keywords: Spatial autocorrelation, MESS, QML, GMM, Heteroskedasticity, Delta method, FDI JEL Classification: C12, C13, C21, F14, F21

\footnotetext{
Email addresses: nicolas.debarsy@univ-orleans.fr (Nicolas Debarsy), jin.fei@shufe.edu.cn (Fei Jin), lee.1777@osu.edu (Lung-fei Lee)

${ }^{1}$ Associate Researcher of the CNRS.
} 


\section{Introduction}

The Matrix Exponential Spatial Specification (MESS) has been initially proposed by LeSage and Pace (2007) as a substitute to the well-known spatial autoregressive (SAR) specification. The difference between the two rests on the type of decay which characterizes the influence of space. The MESS uses an exponential decay while the SAR specification is based on a geometrical decay. The motivation of these authors to use the MESS is its computational simplicity. Indeed, in contrast to the SAR, the quasi-maximum likelihood (QML) function of the MESS does not involve any Jacobian of the transformation and thus reduces to a nonlinear regression estimation. This is so even for its extension to models with a finite number of spatial weights matrices. A second advantage of the MESS is the absence of constraints on the parameter space of the coefficient that captures interactions between observations since the reduced form of the MESS always exists (see Chiu et al., 1996). Furthermore, no positivity constraint on the Jacobian of the transformation needs be imposed as it does not appear in the quasi log-likelihood function. In Section 2, we nevertheless show that MESS and SAR models cannot be seen as perfect substitutes since neither a one-to-one correspondence between the parameters capturing interactions nor between impacts (except in some specific cases) can be derived. Furthermore, a MESS model is always a stable spatial process, but a SAR model with strong spatial interaction might be unstable. ${ }^{2}$

A third advantage of the MESS, proved in this paper, is that the quasi-maximum likelihood estimator (QMLE) is consistent even in the presence of unknown heteroskedasticity, a feature not shared by the SAR model (see Lin and Lee, 2010, p. 36). These two authors have, however, shown that a generalized method of moments (GMM) estimator (GMME) with properly modified quadratic moment conditions in a SAR specification could still be consistent in presence of unknown heteroskedasticity. ${ }^{3}$ Using quadratic moment conditions similar to those in Lin and Lee (2010), we derive an optimal GMME consistent in presence of unknown heteroskedasticity and also generally more efficient with respect to the QMLE (with either normal or non-normal disturbances). The relative efficiency of the optimal GMME results from the optimal weighting of the GMM estimation method which uses the same moments that the QMLE integrates. In the homoskedastic case, we derive a best (optimal) GMME that is as efficient as the MLE under normality and can be more efficient than the QMLE under non-normality. ${ }^{4}$ The best GMME takes a much simpler

\footnotetext{
${ }^{2}$ From this view, we may argue that the MESS would be useful only when observed outcomes do not show unstable phenomena.

${ }^{3}$ Kelejian and Prucha (2010) also develop a GMME robust to the presence of heteroskedasticity but their main focus was on spatial autocorrelation in the error terms.

${ }^{4}$ Lee (2007) derives the best optimal GMME for the SAR model with normal i.i.d. disturbances, which is as efficient as the QMLE. Liu et al. (2010) considers the best optimal GMME for the SAR model with SAR disturbances that can be more efficient than the QMLE under non-normality, which is extended to high order SAR models in Lee and Liu (2010).
} 
form than that for the SAR model and the optimal orthogonal conditions do not involve any estimated parameters ${ }^{5}$.

Even though LeSage and Pace (2007) present the maximum likelihood and Bayesian estimations of the MESS, not any asymptotic theory has been derived for this specification. In this paper, we focus our attention on the general model where a MESS is present in both the dependent variable and in the error terms (MESS(1,1) for short), and develop large sample properties for QML and GMM methods under both homoskedastic and heteroskedastic cases. ${ }^{6}$ In the homoskedastic case, the best GMME for models with normal disturbances or commutative spatial weights matrices in the MESS(1,1) is as efficient as the QMLE but generally more efficient than the QMLE for other ones. In the (unknown) heteroskedastic case, the QMLE for the MESS(1,1) model can be consistent only when the spatial weights matrices for the MESS in the dependent variable and in disturbances are commutative, but it is less efficient than an optimal GMME. If different variances in the heteroskedastic case could be estimated consistently, a best GMME could also be implemented. ${ }^{7}$ We also perform Monte Carlo experiments to assess the small sample performance of our proposed estimators.

Analysis of significance of determinants' causal effects on the dependent variable is of great interest for economists. In this paper, we derive a lemma allowing to perform inference on the matrix of impacts implied by the reduced form of the $\operatorname{MESS}(1,1)$. The lemma is based on an adapted version of the Delta method and can be used to test the significance of (functions of) impact's matrix elements as long as the number of constraints is not depending on the sample size. This lemma is valuable for applied economists since until now, with the exception of LeSage and Pace (2009) who provide inference for scalar summaries of these impacts in the SAR model, there does not exist any statistical test to assess the significance of (functions of) impacts.

The developed estimators are finally applied to a modified gravity equation aimed at explaining Belgium's outward FDI. Blonigen et al. (2007) propose four different classifications of FDI which can be distinguished based on the sign of spatial autocorrelation and market-potential of host countries. In addition to obtaining significant and expected signs for the traditional variables included in the gravity model when spatial autocorrelation is accounted for, namely GDP, population and bilateral distance, we find a significant negative spatial autocorrelation and a positive but non-significant market potential effect for hosts countries.

\footnotetext{
${ }^{5}$ See Lee $(2003,2007)$ for further details.

${ }^{6}$ Appendix A considers the QML estimation of a high order MESS, namely MESS(p,q), with $p$ and $q$ being the orders of the MESS in the dependent variable and in the errors respectively. While the parameter spaces for high order SAR models can be hard to find (Lee and Liu, 2010; Elhorst et al., 2012), high order MESS models have the advantage that the parameter spaces are not restricted.

${ }^{7}$ For the SAR model under unknown heteroskedasticity, Lin and Lee (2010) have not discussed the possible best GMME.
} 
Thus vertical FDI is the dominant type of outward FDI for Belgium. We however find that least squares estimators (which do not account for spatial autocorrelation in FDI) are misleading. We further compare MESS $(1,1)$ and SARAR results and show that their economic conclusions in terms of impacts are very similar. However, the MESS, for several reasons, namely computational, technical and statistical, can be more appealing. Finally, statistical significance on impacts is analyzed through the application of the derived lemma for inference.

The rest of the paper is organized as follows. Section 2 compares MESS and SAR models in a more formal way. Section 3 derives the QML and GMM estimators under both homoskedasticity and unknown heteroskedasticity. It also derives a lemma to perform inference on the elements of the matrix of impacts of explanatory variables obtained from the reduced form of the MESS(1,1). Section 4 presents Monte Carlo experiments while Section 5 presents the application of our estimators and applies the lemma for inference on the determinants of Belgium's outward FDI. Section 6 concludes. Some lemmas and proofs are collected in the appendices. ${ }^{8}$

\section{Comparison of MESS and SAR Specifications}

The MESS in LeSage and Pace (2007) is

$$
e^{\alpha W_{n}} y_{n}=X_{n} \beta+\epsilon_{n}, \quad \epsilon_{n}=\left(\epsilon_{n 1}, \ldots, \epsilon_{n n}\right)^{\prime},
$$

where $n$ is the sample size, $y_{n}$ is the $n$-dimensional vector of observations on the dependent variable, $X_{n}$ is an $n \times k$ matrix of exogenous variables with corresponding coefficient vector $\beta, W_{n}$ is an $n \times n$ spatial weights matrix modeling interactions among observations (with zero diagonal elements), $\epsilon_{n i}$ 's are independent with mean zero, and $\alpha$ is the parameter measuring the intensity of interactions between observations. The matrix exponential $e^{\alpha W_{n}}=\sum_{i=0}^{\infty} \frac{\alpha^{i} W_{n}^{i}}{i !}$, where $A_{n}^{0}$ for an $n \times n$ square matrix $A_{n}$ is defined as the $n \times n$ identity matrix $I_{n}$, is always invertible, with its inverse being $e^{-\alpha W_{n}}$ (Chiu et al., 1996). As a result, the variancecovariance (VC) matrix of $y_{n}$ which equals to $e^{-\alpha_{0} W_{n}} \mathrm{E}\left(\epsilon_{n} \epsilon_{n}^{\prime}\right) e^{-\alpha_{0} W_{n}^{\prime}}$ with $\alpha_{0}$ being the true value of $\alpha$, is always positive definite. No restrictions on the parameter space of $\alpha$ should thus be imposed.

In this paper, we consider a general model that has MESS in both the dependent variable and the disturbances that we label MESS(1,1) (which should be viewed as an analog of the SAR model with SAR

\footnotetext{
${ }^{8}$ Except the proof of Proposition 8 which is presented in Appendix C, proofs of remaining propositions are similar to those in Lee (2004) and Lee (2007). Those proofs are provided in a supplementary file, which is available upon request.
} 
disturbances, i.e., SARAR model):

$$
e^{\alpha W_{n}} y_{n}=X_{n} \beta+u_{n}, \quad e^{\tau M_{n}} u_{n}=\epsilon_{n}, \quad \epsilon_{n}=\left(\epsilon_{n 1}, \ldots, \epsilon_{n n}\right)^{\prime},
$$

where $W_{n}$ and $M_{n}$ are $n \times n$ spatial weights matrices. The $M_{n}$ may or may not be different from $W_{n}$. For purposes of comparison and later reference, we put down the SARAR model with the same $W_{n}, M_{n}, X_{n}$, $y_{n}$ and $\epsilon_{n}$ :

$$
\left(I_{n}-\lambda W_{n}\right) y_{n}=X_{n} \beta+u_{n}, \quad\left(I_{n}-\rho M_{n}\right) u_{n}=\epsilon_{n} .
$$

The parameter spaces of $\lambda$ and $\rho$ should be restricted so that the VC matrix of $y_{n}$, namely $\left(I_{n}-\lambda W_{n}\right)^{-1}\left(I_{n}-\right.$ $\left.\rho M_{n}\right)^{-1} \mathrm{E}\left(\epsilon_{n} \epsilon_{n}^{\prime}\right)\left(I_{n}-\rho M_{n}^{\prime}\right)^{-1}\left(I_{n}-\lambda W_{n}^{\prime}\right)^{-1}$ exists. For the SARAR model with a row-normalized $W_{n}$ matrix, the parameter space for $\lambda$ is typically considered to be $(-1,1){ }^{9}$

The quasi log likelihood function of the $\operatorname{MESS}(1,1)$ presented in $(2)$, as if the $\epsilon_{n i}$ 's were i.i.d. normal, is

$$
L_{n}(\theta)=-\frac{n}{2} \ln \left(2 \pi \sigma^{2}\right)+\ln \left|e^{\alpha W_{n}}\right|+\ln \left|e^{\tau M_{n}}\right|-\frac{1}{2 \sigma^{2}}\left(e^{\alpha W_{n}} y_{n}-X_{n} \beta\right)^{\prime} e^{\tau M_{n}^{\prime}} e^{\tau M_{n}}\left(e^{\alpha W_{n}} y_{n}-X_{n} \beta\right),
$$

where $\theta=\left(\gamma^{\prime}, \sigma^{2}\right)^{\prime}$ with $\gamma=\left(\alpha, \tau, \beta^{\prime}\right)^{\prime}$. Let $\theta_{0}$ be the true parameter vector. Since $\left|e^{\alpha W_{n}}\right|=e^{\alpha \operatorname{tr}\left(W_{n}\right)}$ and $\left|e^{\tau M_{n}}\right|=e^{\tau \operatorname{tr}\left(M_{n}\right)}$, as long as $W_{n}$ and $M_{n}$ have zero diagonals, the Jacobian of the transformation disappears and the quasi log likelihood function is simplified to

$$
L_{n}(\theta)=-\frac{n}{2} \ln \left(2 \pi \sigma^{2}\right)-\frac{1}{2 \sigma^{2}}\left(e^{\alpha W_{n}} y_{n}-X_{n} \beta\right)^{\prime} e^{\tau M_{n}^{\prime}} e^{\tau M_{n}}\left(e^{\alpha W_{n}} y_{n}-X_{n} \beta\right)
$$

By contrast, the quasi log likelihood function of the SARAR model shown in (3) involves the log determinant of the Jacobian $\ln \left|\left(I_{n}-\lambda W_{n}\right)\left(I_{n}-\rho M_{n}\right)\right|=\ln \left|I_{n}-\lambda W_{n}\right|+\ln \left|I_{n}-\rho M_{n}\right|$, which may make the QMLE computationally intensive for large sample sizes.

Another difference between these two specifications is that one does not need to normalize the interaction matrices in the MESS. In the SARAR model, the purpose of normalizing the interaction matrices is to standardize the parameter spaces for $\lambda$ and $\rho$ so that they correspond to $(-1,1)$, which facilitates the interpretation of these parameters. However, in the MESS, since no parameter constraint is involved, the

\footnotetext{
${ }^{9}$ See Kelejian and Prucha (2010) for a detailed discussion about the parameter space for $\lambda$. For high order SARAR models, finding the parameter spaces can be hard. Elhorst et al. (2012) have outlined a procedure for finding the stationary region, but the parameter spaces can be complicated even for a SAR model with two spatial weights matrices for the dependent variable. By contrast, parameters in high order MESS models, labeled $\operatorname{MESS}(p, q)$, where $p$ and $q$ are the orders of the MESS in the dependent variable and disturbances respectively, do not need to be restricted and the effort to find the parameter spaces is saved. Appendix A considers the QML estimation of these high order MESS models.
} 
normalization of the interaction matrices may not play a special role.

LeSage and Pace (2007) present the MESS as a computationally simpler substitute for the SAR model. Using a row-normalized interaction matrix $W_{n}$, they propose the approximated relation $\lambda=1-e^{\alpha}$. They argue that this approximation is derived by equating the length of $\left\|e^{\alpha W_{n}}\right\|_{\infty}$ and $\left\|I_{n}-\lambda W_{n}\right\|_{\infty}$, with $\|.\|_{\infty}$ being the maximum row sum matrix norm. However, this approximation is not always right since the matrix norm should be taken over the absolute value of matrix elements. By contrast, if one turns to the impact analysis, an equivalence between the two specifications can be traced back at least in some specific cases.

Before presenting this correspondence, it is important to discuss the features of impact analysis in spatial autoregressive (SAR or MESS) regressions. Impact analysis, which is one of the main focuses for economists, is based on the reduced form of the estimated econometric specification. For the $\operatorname{MESS}(1,1)$ case, the reduced form is

$$
y_{n}=e^{-\alpha W_{n}}\left(X_{n} \beta+e^{-\tau M_{n}} \epsilon_{n}\right) .
$$

One then computes the matrix of impact for each regressor $X_{n k}, k=1, \cdots, k$, by calculating the partial derivative of $y_{n}$ with respect to the concerned regressor. For a continuous regressor $X_{n k}$, this matrix is

$$
\Xi_{X_{n k}}^{y_{n}}=\frac{\partial y_{n}}{\partial X_{n k}^{\prime}}=\beta_{k} e^{-\alpha W_{n}} .
$$

The diagonal elements of this matrix contain the direct effects including own-spillover effects, which are inherently heterogeneous in presence of spatial autocorrelation due to differentiated friction terms in the interaction matrix. This is what Debarsy and Ertur (2010) call interactive heterogeneity. Off-diagonal elements of this matrix represent indirect effects, meaning the impact of a change in explanatory variable for individual $j$ on the dependent variable for individual $i$. These direct and indirect effects are presented in the following expressions:

$$
\begin{aligned}
\frac{\partial y_{n, i}}{\partial X_{n k, i}} & \equiv\left(\Xi_{X_{n k}}^{y_{n}}\right)_{i i}, \\
\frac{\partial y_{n, i}}{\partial X_{n k, j}} & \equiv\left(\Xi_{X_{n k}}^{y_{n}}\right)_{i j} .
\end{aligned}
$$

For the SARAR model, its associated reduced form is

$$
y_{n}=\left(I_{n}-\lambda W_{n}\right)^{-1}\left[X_{n} \beta+\left(I_{n}-\rho M_{n}\right)^{-1} \epsilon_{n}\right],
$$


and the implied impact matrix for regressor $X_{n k}$ is

$$
\Xi_{X_{n k}}^{y_{n}}=\frac{\partial y_{n}}{\partial X_{n k}^{\prime}}=\beta_{k}\left(I_{n}-\lambda W_{n}\right)^{-1}
$$

To summarize the information conveyed by these matrices of impacts, LeSage and Pace (2009) propose extracting several scalar measures, as the average direct effect (mean of the diagonal elements), average total effect (average of the row or column sums) and average indirect effect (average of the column or row sums excluding the diagonal element).

Consider a row-normalized interaction matrix $W_{n}$ in the $\operatorname{MESS}(1,1)$ model. Suppose that a shock of the same magnitude $\Delta x$ is applied on the $k$ th explanatory variable $X_{n k}$ to all spatial units. The new explanatory variable is now $X_{n k}+l_{n} \Delta x$, with $l_{n}$ being the $n$-dimensional vector of ones. For the $\operatorname{MESS}(1,1)$, from its reduced form (5), one calculates a total impact of $\Delta y_{n}=e^{-\alpha W_{n}} l_{n} \Delta x \beta_{k}$. The average total effect is thus equal to $\frac{1}{n} l_{n}^{\prime} \Delta y_{n}=e^{-\alpha} \Delta x \beta_{k} \cdot{ }^{10}$ Correspondingly, the average total impact of $X_{n k}$ in the SARAR model is $\frac{1}{n} l_{n}^{\prime} \Delta y_{n}=\frac{1}{1-\lambda} \Delta x \beta_{k}$. Equating the two gives the relation $\alpha=\ln (1-\lambda)$ or $\lambda=1-e^{\alpha}$. Thus, there is a negative relation between $\lambda$ and $\alpha$. At $\lambda=0, \alpha=0$ and, when $0<\lambda<1, \alpha$ will take on negative values and vice-versa. For an interaction matrix $W_{n}$ that is not row-normalized, there is no such a relation.

Even though a relation between $\lambda$ and $\alpha$ can be found for a row-normalized $W_{n}$, we nevertheless cannot consider that these two models as substitutes of each other. The underlying reason lies in the comparison of parameter spaces. As mentioned above, for the SARAR model with row-normalized $W_{n}, \lambda$ is usually restricted to the range $(-1,1)$. However, in the $\operatorname{MESS}(1,1), \alpha \in(-\infty, \infty)$. So, in the case of rownormalized $W_{n}$, while $\lambda \leq-1$ is not allowed for a SARAR model, $\alpha \geq \ln (2)$ can be valid, meaning that parameter spaces of $\alpha$ and $\lambda$ do not correspond. So, for high negative spatial autocorrelation, we could observe substantial difference between these two models. ${ }^{11}$ Furthermore, in a SAR model, if $\lambda>1$, it would be an unstable model, while unstability does not occur for the MESS with any finite value of $\alpha$.

\footnotetext{
${ }^{10} \mathrm{As} W_{n}$ is row-normalized, $W_{n}^{k} l_{n}=W_{n} l_{n}=l_{n}, k \in \mathbb{N}$.

${ }^{11}$ For a non-negative and row-normalized interaction matrix $W_{n}$, the parameter space for $\lambda$ may be taken as the interval $\left(\frac{1}{\mu_{\min , n}}, 1\right)$ with $\mu_{\min , n}$ being the minimal real eigenvalue of $W_{n}$. However, it does not change the conclusions regarding the difference between parameter spaces for $\lambda$ and $\alpha$.
} 


\section{Estimations of the MESS(1,1) Model}

We consider the QML estimation as well as the GMM estimation of the MESS(1,1) in this section. From (4), it is apparent that the QMLE of $\gamma$ is the minimizer of the function

$$
Q_{n}(\gamma)=\left(e^{\alpha W_{n}} y_{n}-X_{n} \beta\right)^{\prime} e^{\tau M_{n}^{\prime}} e^{\tau M_{n}}\left(e^{\alpha W_{n}} y_{n}-X_{n} \beta\right)
$$

The derivatives of $Q_{n}(\gamma)$ with respect to $\alpha, \tau$ and $\beta$ at $\gamma_{0}$ are, respectively,

$$
\begin{aligned}
& \frac{\partial Q_{n}\left(\gamma_{0}\right)}{\partial \alpha}=2\left(X_{n} \beta_{0}+e^{-\tau_{0} M_{n}} \epsilon_{n}\right)^{\prime} W_{n}^{\prime} e^{\tau_{0} M_{n}^{\prime}} \epsilon_{n}, \\
& \frac{\partial Q_{n}\left(\gamma_{0}\right)}{\partial \tau}=2 \epsilon_{n}^{\prime} M_{n} \epsilon_{n}, \\
& \frac{\partial Q_{n}\left(\gamma_{0}\right)}{\partial \beta}=-2 X_{n}^{\prime} e^{\tau_{0} M_{n}^{\prime}} \epsilon_{n} .
\end{aligned}
$$

When $\epsilon_{n i}$ 's are i.i.d. with mean zero and variance $\sigma_{0}^{2}$, as $\mathrm{E}\left(\epsilon_{n}^{\prime} M_{n} \epsilon_{n}\right)=\operatorname{tr}\left[M_{n} \mathrm{E}\left(\epsilon_{n} \epsilon_{n}^{\prime}\right)\right]=\sigma_{0}^{2} \operatorname{tr}\left(M_{n}\right)=0$ and $\mathrm{E}\left(\epsilon_{n}^{\prime} e^{-\tau_{0} M_{n}^{\prime}} W_{n}^{\prime} e^{\tau_{0} M_{n}^{\prime}} \epsilon_{n}\right)=\sigma_{0}^{2} \operatorname{tr}\left(W_{n}^{\prime} e^{\tau_{0} M_{n}^{\prime}} e^{-\tau_{0} M_{n}^{\prime}}\right)=\sigma_{0}^{2} \operatorname{tr}\left(W_{n}^{\prime}\right)=0$, the expected value of $\frac{\partial Q_{n}\left(\gamma_{0}\right)}{\partial \gamma}$ is zero, which verifies that the minimizer of $\mathrm{E} Q_{n}(\gamma)$ can be $\gamma_{0}$. When $\epsilon_{n i}$ 's are independent with mean zero but different variances $\sigma_{n i}^{2}$ 's, $\mathrm{E}\left(\epsilon_{n}^{\prime} M_{n} \epsilon_{n}\right)=\operatorname{tr}\left(M_{n} \Sigma_{n}\right)=0$ since the diagonal elements of $M_{n}$ are all zero, and $\Sigma_{n}=\operatorname{Diag}\left(\sigma_{n 1}^{2}, \ldots, \sigma_{n n}^{2}\right)$ is a diagonal matrix containing the different variances as diagonal elements. In addition, $\mathrm{E}\left(\epsilon_{n}^{\prime} e^{-\tau_{0} M_{n}^{\prime}} W_{n}^{\prime} e^{\tau_{0} M_{n}^{\prime}} \epsilon_{n}\right)=\operatorname{tr}\left(e^{-\tau_{0} M_{n}^{\prime}} W_{n}^{\prime} e^{\tau_{0} M_{n}^{\prime} \Sigma_{n}}\right)$, which may not be zero in general. But if $W_{n} M_{n}=M_{n} W_{n}$, then $W_{n}^{\prime} e^{\tau_{0} M_{n}^{\prime}}=e^{\tau_{0} M_{n}^{\prime}} W_{n}^{\prime}$ and $\mathrm{E}\left(\epsilon_{n}^{\prime} e^{-\tau_{0} M_{n}^{\prime}} W_{n}^{\prime} e^{\tau_{0} M_{n}^{\prime}} \epsilon_{n}\right)=\operatorname{tr}\left(W_{n}^{\prime} \Sigma_{n}\right)=0$. Therefore, when the matrix $W_{n}$ in the spatial lag process can be commutative with the matrix $M_{n}$ in the spatial error process, the QMLE for $\gamma$, derived from the minimization of $Q_{n}(\gamma)$, can be consistent even under unknown heteroskedasticity. This includes the special cases that there is no MESS process in the disturbances or that $M_{n}=W_{n}$. This robustness of the QMLE for the $\operatorname{MESS}(1,0)$ and $\operatorname{MESS}(1,1)$ to unknown heteroskedasticity is a nice feature not shared by the QMLE for the SARAR model.

The function to be minimized, namely (11), may be written as

$$
Q_{n}(\gamma)=\left(y_{n}-e^{-\alpha W_{n}} X_{n} \beta\right)^{\prime}\left(e^{-\alpha W_{n}} e^{-\tau M_{n}} e^{-\tau M_{n}^{\prime}} e^{-\alpha W_{n}^{\prime}}\right)^{-1}\left(y_{n}-e^{-\alpha W_{n}} X_{n} \beta\right)
$$

Using the reduced form of the $\operatorname{MESS}(1,1)$, namely $y_{n}=e^{-\alpha_{0} W_{n}}\left(X_{n} \beta_{0}+e^{-\tau_{0} M_{n}} \epsilon_{n}\right)$, and assuming that $\mathrm{E}\left(\epsilon_{n} \epsilon_{n}^{\prime}\right)=\sigma_{0}^{2} I_{n}$, the VC matrix of $y_{n}$ is $\sigma_{0}^{2} e^{-\alpha_{0} W_{n}} e^{-\tau_{0} M_{n}} e^{-\tau_{0} M_{n}^{\prime}} e^{-\alpha_{0} W_{n}^{\prime}}$ and the QMLE can be seen as a continuously updating version of the generalized nonlinear least squares (GNLS). The similarity between 
the QML and GNLS is due to the special structure of the matrix exponential specification. By contrast, there is no such a similarity for the SARAR model (3). ${ }^{12}$

In addition to the QML estimation, we may also consider the GMM estimation of the MESS $(1,1)$ using both linear and quadratic moments, as for the SARAR model. The linear moments would be of the form $F_{n}^{\prime} \epsilon_{n}(\gamma)=0$, where $\epsilon_{n}(\gamma)=e^{\tau M_{n}}\left(e^{\alpha W_{n}} y_{n}-X_{n} \beta\right)$ and $F_{n}$ is an $n \times k_{f}$ matrix of instruments constructed as functions of $W_{n}$ and $X_{n}$, as in the two-stage least squares (2SLS) approach. The quadratic moments have the form $\epsilon_{n}^{\prime}(\gamma) P_{n i} \epsilon_{n}(\gamma)=0 ; i=1, \cdots, k_{p}$, where $P_{n i}$ has trace zero when $\epsilon_{n i}$ 's are i.i.d., implying that $\mathrm{E}\left[\epsilon_{n}^{\prime}\left(\gamma_{0}\right) P_{n i} \epsilon_{n}\left(\gamma_{0}\right)\right]=\sigma_{0}^{2} \operatorname{tr}\left(P_{n i}\right)=0$. On the other hand, if the diagonal elements of $P_{n i}$ are all zero when $\epsilon_{n i}$ 's are independently distributed with possibly different variances, we get $\mathrm{E}\left[\epsilon_{n}^{\prime}\left(\gamma_{0}\right) P_{n i} \epsilon_{n}\left(\gamma_{0}\right)\right]=$ $\operatorname{tr}\left(P_{n i} \Sigma_{n}\right)=0$.

The basic regularity conditions for estimation are assumed below. The specific sets of hypotheses required for both methods will be given subsequently.

Assumption 1. Matrices $\left\{W_{n}\right\}$ and $\left\{M_{n}\right\}$ are bounded in both row and column sum norms. The diagonal elements of $W_{n}$ and $M_{n}$ are zero.

Assumption 2. Elements of $X_{n}$ are uniformly bounded constants, $X_{n}$ has full column rank, and besides, $\lim _{n \rightarrow \infty} X_{n}^{\prime} X_{n} / n$ exists and is nonsingular.

Assumptions 1 and 2 follow from the literature, see, e.g., Kelejian and Prucha (1998) and Lee (2004).

\section{1. $Q M L E$}

For the QMLE from (4), we may just investigate the minimizers of the functions $\left\{Q_{n}(\gamma)\right\}$. For a fixed $\phi=(\alpha, \tau)^{\prime}$, minimizing $Q_{n}(\gamma)$ yields

$$
\hat{\beta}_{n}(\phi)=\left(X_{n}^{\prime} e^{\tau M_{n}^{\prime}} e^{\tau M_{n}} X_{n}\right)^{-1} X_{n}^{\prime} e^{\tau M_{n}^{\prime}} e^{\tau M_{n}} e^{\alpha W_{n}} y_{n}
$$

Substituting $\hat{\beta}_{n}(\phi)$ into $Q_{n}(\gamma)$, we obtain a function of only $\phi$ :

$$
Q_{n}(\phi)=y_{n}^{\prime} e^{\alpha W_{n}^{\prime}} e^{\tau M_{n}^{\prime}} H_{n}(\tau) e^{\tau M_{n}} e^{\alpha W_{n}} y_{n}
$$

\footnotetext{
${ }^{12} \mathrm{~A}$ function for the SARAR model with a structure similar to $Q_{n}(\gamma)$ is $Q_{n}^{*}(\psi)=\left[\left(I_{n}-\lambda W_{n}\right) y_{n}-X_{n} \beta\right]^{\prime}\left(I_{n}-\rho M_{n}^{\prime}\right)\left(I_{n}-\right.$ $\left.\rho M_{n}\right)\left[\left(I_{n}-\lambda W_{n}\right) y_{n}-X_{n} \beta\right]$ with $\psi=\left(\lambda, \rho, \beta^{\prime}\right)^{\prime}$. At $\psi_{0}, \mathrm{E}\left(\frac{\partial Q_{n}^{*}\left(\psi_{0}\right)}{\partial \lambda}\right)=-2 \sigma_{0}^{2} \operatorname{tr}\left[W_{n}\left(I_{n}-\lambda_{0} W_{n}\right)^{-1}\right]$ and $\mathrm{E}\left(\frac{\partial Q_{n}^{*}\left(\psi_{0}\right)}{\partial \rho}\right)=$ $-2 \sigma_{0}^{2} \operatorname{tr}\left[M_{n}\left(I_{n}-\rho_{0} M_{n}\right)^{-1}\right]$, where $\operatorname{tr}\left[W_{n}\left(I_{n}-\lambda_{0} W_{n}\right)^{-1}\right] \neq 0$ and $\operatorname{tr}\left[M_{n}\left(I_{n}-\rho_{0} M_{n}\right)^{-1}\right] \neq 0$ in general. Thus the minimizer of $Q_{n}^{*}(\psi)$ is not expected to be a consistent estimator of $\psi$ in the SARAR model (3).
} 
where $H_{n}(\tau)=I_{n}-e^{\tau M_{n}} X_{n}\left(X_{n}^{\prime} e^{\tau M_{n}^{\prime}} e^{\tau M_{n}} X_{n}\right)^{-1} X_{n}^{\prime} e^{\tau M_{n}^{\prime}}$ is a projection matrix. The function $Q_{n}(\phi)$ can be used for the analysis of the consistency of the QMLE. Although we may not need to restrict the parameter space of $\phi$ in practice, $\phi$ should be bounded in analysis so that $e^{\alpha W_{n}}$ and $e^{\tau M_{n}}$ would be bounded in both row and column sum norms, since $\left\|e^{\alpha W_{n}}\right\|=\left\|\sum_{i=0}^{\infty} \frac{\alpha^{i} W_{n}^{i}}{i !}\right\| \leq \sum_{i=0}^{\infty} \frac{|\alpha|^{i}\left\|W_{n}\right\|^{i}}{i !}=e^{|\alpha| \cdot\left\|W_{n}\right\|}$, which is bounded if $\alpha$ is bounded, and so is $\left\|e^{\tau M_{n}}\right\|$ if $\tau$ is bounded, where $\|\cdot\|$ denotes either the row or column sum matrix norm.

Assumption 3. There exists a constant $\delta>0$ such that $|\alpha| \leq \delta,|\tau| \leq \delta$ and the true $\phi_{0}$ is in the interior of the parameter space $\Phi=[-\delta, \delta] \times[-\delta, \delta]$.

For consistency of the QMLE, we need to show that the difference between $Q_{n}(\phi) / n$ and some nonstochastic function $\bar{Q}_{n}(\phi) / n$ converges to zero uniformly over the parameter space $\Phi .{ }^{13}$ The $\bar{Q}_{n}(\phi)$ will have different forms in the homoskedastic and heteroskedastic cases. By Assumptions 2 and $3, \frac{1}{n} X_{n}^{\prime} e^{\tau M_{n}^{\prime}} e^{\tau M_{n}} X_{n}$ is bounded. The $Q_{n}(\phi)$ is a well-defined function for large enough $n$ if the limit of $\frac{1}{n} X_{n}^{\prime} e^{\tau M_{n}^{\prime}} e^{\tau M_{n}} X_{n}$ exists and is nonsingular. In addition, we require that the sequence of the smallest eigenvalues of $e^{\tau M_{n}^{\prime}} e^{\tau M_{n}}$ be bounded away from zero uniformly in $\tau$, so that $H_{n}(\tau)$ is bounded in both row and column sum norms uniformly in $\tau$. As $e^{\tau M_{n}^{\prime}} e^{\tau M_{n}}$ is positive definite, its smallest eigenvalue is positive. The condition further limits all the eigenvalues to be strictly positive uniformly over the parameter space for all $n$.

Assumption 4. The limit $\lim _{n \rightarrow \infty} \frac{1}{n} X_{n}^{\prime} e^{\tau M_{n}^{\prime}} e^{\tau M_{n}} X_{n}$ exists and is nonsingular for any $\tau \in[-\delta, \delta]$, and the sequence of the smallest eigenvalues of $e^{\tau M_{n}^{\prime}} e^{\tau M_{n}}$ is bounded away from zero uniformly in $\tau \in[-\delta, \delta]$.

\subsubsection{QMLE: Homoskedastic Case}

In this part, we establish the consistency and asymptotic normality of the QMLE for the MESS $(1,1)$ with i.i.d. disturbances.

Assumption 5. The $\epsilon_{n i}$ 's are i.i.d. with mean zero and variance $\sigma_{0}^{2}$ and the moment $\mathrm{E}\left|\epsilon_{n i}\right|^{4+\eta}$ for some $\eta>0$ exists.

Define $\bar{Q}_{n}(\phi)=\min _{\beta} \mathrm{E} Q_{n}(\gamma)$, then

$$
\begin{aligned}
\bar{Q}_{n}(\phi)= & \left(X_{n} \beta_{0}\right)^{\prime} e^{\left(\alpha-\alpha_{0}\right) W_{n}^{\prime}} e^{\tau M_{n}^{\prime}} H_{n}(\tau) e^{\tau M_{n}} e^{\left(\alpha-\alpha_{0}\right) W_{n}} X_{n} \beta_{0} \\
& +\sigma_{0}^{2} \operatorname{tr}\left(e^{-\tau_{0} M_{n}^{\prime}} e^{\left(\alpha-\alpha_{0}\right) W_{n}^{\prime}} e^{\tau M_{n}^{\prime}} e^{\tau M_{n}} e^{\left(\alpha-\alpha_{0}\right) W_{n}} e^{-\tau_{0} M_{n}}\right) .
\end{aligned}
$$

\footnotetext{
${ }^{13}$ The main purpose for Assumption 3 is to guarantee that uniform convergence of relevant objects is possible on a compact parameter space.
} 
The identification of $\phi_{0}$ can be based on the minimum values of $\left\{\bar{Q}_{n}(\phi) / n\right\}$. To ensure the identification uniqueness, the following condition is assumed.

Assumption 6. Either (i) $\lim _{n \rightarrow \infty} n^{-1}\left(X_{n} \beta_{0}\right)^{\prime} e^{\left(\alpha-\alpha_{0}\right) W_{n}^{\prime}} e^{\tau M_{n}^{\prime}} H_{n}(\tau) e^{\tau M_{n}} e^{\left(\alpha-\alpha_{0}\right) W_{n}} X_{n} \beta_{0} \neq 0$ for any $\tau$ and $\alpha \neq \alpha_{0}$, and $\lim _{n \rightarrow \infty} n^{-1} \operatorname{tr}\left(e^{\left(\tau-\tau_{0}\right) M_{n}^{\prime}} e^{\left(\tau-\tau_{0}\right) M_{n}}\right)>1$ for any $\tau \neq \tau_{0}$, or

(ii) $\lim _{n \rightarrow \infty} n^{-1} \operatorname{tr}\left(e^{-\tau_{0} M_{n}^{\prime}} e^{\left(\alpha-\alpha_{0}\right) W_{n}^{\prime}} e^{\tau M_{n}^{\prime}} e^{\tau M_{n}} e^{\left(\alpha-\alpha_{0}\right) W_{n}} e^{-\tau_{0} M_{n}}\right)>1$ for any $\phi \neq \phi_{0}$.

The identification of $\alpha_{0}$ can come from the first term on the r.h.s. of (17). As $H_{n}(\tau) e^{\tau M_{n}} X_{n}=0$, the first term at $\alpha_{0}$ is zero for any $\tau$. Thus the first term is not sufficient to identify $\tau_{0}$. Given the identification of $\alpha_{0}, \tau_{0}$ can be identified from the second term. As $\lim _{n \rightarrow \infty} n^{-1} X_{n}^{\prime} e^{\tau M_{n}^{\prime}} e^{\tau M_{n}} X_{n}$ is nonsingular, by the partition matrix formula, $\lim _{n \rightarrow \infty} n^{-1}\left(X_{n} \beta_{0}\right)^{\prime} e^{\left(\alpha-\alpha_{0}\right) W_{n}^{\prime}} e^{\tau M_{n}^{\prime}} H_{n}(\tau) e^{\tau M_{n}} e^{\left(\alpha-\alpha_{0}\right) W_{n}} X_{n} \beta_{0}$ is non-zero if and only if $\lim _{n \rightarrow \infty} n^{-1}\left(X_{n}, e^{\left(\alpha-\alpha_{0}\right) W_{n}} X_{n} \beta_{0}\right)^{\prime} e^{\tau M_{n}^{\prime}} e^{\tau M_{n}}\left(X_{n}, e^{\left(\alpha-\alpha_{0}\right) W_{n}} X_{n} \beta_{0}\right)$ is nonsingular. Thus, the first part of (i) in Assumption 6 relates to asymptotic non-multicollinearity of $e^{\left(\alpha-\alpha_{0}\right) W_{n}} X_{n} \beta_{0}$ with $X_{n}$. In the proof of Proposition 1, it is shown by the inequality of arithmetic and geometric means that $n^{-1} \operatorname{tr}\left(e^{\left(\tau-\tau_{0}\right) M_{n}^{\prime}} e^{\left(\tau-\tau_{0}\right) M_{n}}\right) \geq 1$ holds for any $\tau$. The second part of (i) further requires $n^{-1} \operatorname{tr}\left(e^{\left(\tau-\tau_{0}\right) M_{n}^{\prime}} e^{\left(\tau-\tau_{0}\right) M_{n}}\right)$ to be strictly greater than 1 in the limit when $\tau \neq \tau_{0}$. For a finite $n$, the arithmetic and geometric means are equal if and only if all the eigenvalues of $e^{\left(\tau-\tau_{0}\right) M_{n}^{\prime}} e^{\left(\tau-\tau_{0}\right) M_{n}}$ are equal to each other, which implies that $e^{\left(\tau-\tau_{0}\right) M_{n}^{\prime}} e^{\left(\tau-\tau_{0}\right) M_{n}}$ is proportional to $I_{n}$. This assumption rules out this possibility in the limit whenever $\tau \neq \tau_{0}$. The identification of $\phi_{0}$ can only come from the second term on the r.h.s. of (17), which is given in (ii) of Assumption 6. This relates to the uniqueness of the VC matrix of $y_{n}$, namely $\sigma_{0}^{2} e^{-\alpha_{0} W_{n}} e^{-\tau_{0} M_{n}} e^{-\tau_{0} M_{n}^{\prime}} e^{-\alpha_{0} W_{n}^{\prime}}$, since

$$
\begin{aligned}
& \operatorname{tr}\left(e^{-\tau_{0} M_{n}^{\prime}} e^{\left(\alpha-\alpha_{0}\right) W_{n}^{\prime}} e^{\tau M_{n}^{\prime}} e^{\tau M_{n}} e^{\left(\alpha-\alpha_{0}\right) W_{n}} e^{-\tau_{0} M_{n}}\right) \\
& =\operatorname{tr}\left[e^{-\alpha_{0} W_{n}} e^{-\tau_{0} M_{n}} e^{-\tau_{0} M_{n}^{\prime}} e^{-\alpha_{0} W_{n}^{\prime}}\left(e^{-\alpha W_{n}} e^{-\tau M_{n}} e^{-\tau M_{n}^{\prime}} e^{-\alpha W_{n}^{\prime}}\right)^{-1}\right] .
\end{aligned}
$$

It is obvious that Assumption 6 (i) fails to hold when $\beta_{0}=0$. In this case, the identification will rely solely on (ii). Another case of the failure of (i) even if $\beta_{0} \neq 0$ occurs is when $X_{n}$ contains only an intercept term, i.e., $X_{n}=l_{n}$, and $W_{n}$ is row-normalized. In this case, $H_{n}(\tau) e^{\tau M_{n}} l_{n}=0$. Other cases might be due to very special structures on $W_{n}$ or $M_{n}$. For example, elements of $W_{n}$ and $M_{n}$ except the diagonal ones are all equal to a constant and $X_{n}$ contains an intercept term. Let $W_{n}=M_{n}=(n-1)^{-1}\left(l_{n} l_{n}^{\prime}-I_{n}\right)$ for instance. Then $H_{n}(\tau) e^{\tau M_{n}} W_{n}^{k}=(-1)^{k}(n-1)^{-k} H_{n}(\tau) e^{\tau M_{n}}$. By the expansion form of $e^{\left(\alpha-\alpha_{0}\right) W_{n}}$, $H_{n}(\tau) e^{\tau M_{n}} e^{\left(\alpha-\alpha_{0}\right) W_{n}} X_{n}=e^{\left(\alpha_{0}-\alpha\right) /(n-1)} H_{n}(\tau) e^{\tau M_{n}} X_{n}=0$. Thus the first part in (i) does not hold. Furthermore, since the eigenvalues of $M_{n}$ are $(1-n)^{-1}, \ldots,(1-n)^{-1}$ and 1 , it follows that $M_{n}^{k}$ has eigenvalues 
$(1-n)^{-k}, \ldots,(1-n)^{-k}, 1$. Hence, with this symmetric $M_{n}$,

$$
\begin{aligned}
\frac{1}{n} \operatorname{tr}\left(e^{\left(\tau-\tau_{0}\right) M_{n}^{\prime}} e^{\left(\tau-\tau_{0}\right) M_{n}}\right) & =\frac{1}{n} \operatorname{tr}\left(e^{2\left(\tau-\tau_{0}\right) M_{n}}\right)=\frac{1}{n} \sum_{k=0}^{\infty} \frac{2^{k}\left(\tau-\tau_{0}\right)^{k} \operatorname{tr}\left(M_{n}^{k}\right)}{k !} \\
& =\frac{1}{n} \sum_{k=0}^{\infty} \frac{2^{k}\left(\tau-\tau_{0}\right)^{k}\left[1+(n-1)(1-n)^{-k}\right]}{k !} \\
& =\frac{1}{n} e^{2\left(\tau-\tau_{0}\right)}+\frac{n-1}{n} e^{2\left(\tau-\tau_{0}\right) /(1-n)},
\end{aligned}
$$

which is equal to 1 in the limit. Then the second part in (i) does not hold either. In this case, $\lim _{n \rightarrow \infty} \frac{1}{n} \bar{Q}_{n}(\phi)$ is equal to $\sigma_{0}^{2}$ for any $\phi$. Looking into $Q_{n}(\phi)$ directly, we have

$$
Q_{n}(\phi)=e^{-2 \alpha /(n-1)} y_{n}^{\prime} e^{\tau M_{n}^{\prime}} H_{n}(\tau) e^{\tau M_{n}} y_{n},
$$

which is monotonically decreasing in $\alpha$. Then the QMLE of $\alpha$ will diverge to positive infinity, which is not equal to $\alpha_{0} \cdot{ }^{14}$

In general, (ii) in Assumption 6 would not hold as long as $W_{n}$ and $M_{n}$ are equal. When $M_{n}=W_{n}$,

$$
\operatorname{tr}\left(e^{-\tau_{0} M_{n}^{\prime}} e^{\left(\alpha-\alpha_{0}\right) W_{n}^{\prime}} e^{\tau M_{n}^{\prime}} e^{\tau M_{n}} e^{\left(\alpha-\alpha_{0}\right) W_{n}} e^{-\tau_{0} M_{n}}\right)=\operatorname{tr}\left(e^{\left(\alpha+\tau-\alpha_{0}-\tau_{0}\right) W_{n}^{\prime}} e^{\left(\alpha+\tau-\alpha_{0}-\tau_{0}\right) W_{n}}\right) .
$$

As long as $\alpha+\tau=\alpha_{0}+\tau_{0}, \frac{1}{n} \operatorname{tr}\left(e^{-\tau_{0} M_{n}^{\prime}} e^{\left(\alpha-\alpha_{0}\right) W_{n}^{\prime}} e^{\tau M_{n}^{\prime}} e^{\tau M_{n}} e^{\left(\alpha-\alpha_{0}\right) W_{n}} e^{-\tau_{0} M_{n}}\right)=1$. So for the case that $M_{n}=W_{n}$, the parameter identification depends crucially on Assumption 6 (i). This situation is apparent as the model becomes $y_{n}=e^{-\alpha_{0} W_{n}} X_{n} \beta_{0}+e^{-\left(\alpha_{0}+\tau_{0}\right) W_{n}} \epsilon_{n}$. Thus, when there are no exogenous variables and $W_{n}=M_{n}$ in the $\operatorname{MESS}(1,1), \alpha_{0}$ and $\tau_{0}$ cannot be identified.

With the identification uniqueness and uniform convergence of $\left[Q_{n}(\phi)-\bar{Q}_{n}(\phi)\right] / n$ to zero on the parameter space $\Phi$, the consistency of the QMLE follows.

Proposition 1. Under Assumptions 1-6, the QMLE $\hat{\gamma}_{n}$ of the MESS(1,1) is consistent.

The asymptotic distribution of $\hat{\gamma}_{n}$ can be derived from applying a Taylor expansion to the first-order condition $\frac{\partial Q_{n}\left(\hat{\gamma}_{n}\right)}{\partial \gamma}=0$ at the true $\gamma_{0}$ :

$$
\frac{\partial Q_{n}\left(\hat{\gamma}_{n}\right)}{\partial \gamma}=0=\frac{\partial Q_{n}\left(\gamma_{0}\right)}{\partial \gamma}+\frac{\partial^{2} Q_{n}\left(\tilde{\gamma}_{n}\right)}{\partial \gamma \partial \gamma^{\prime}}\left(\hat{\gamma}_{n}-\gamma_{0}\right)
$$

\footnotetext{
${ }^{14}$ See Smith (2009) for a discussion of this special case in the SAR model.
} 
where $\tilde{\gamma}_{n}$ is between $\hat{\gamma}_{n}$ and $\gamma_{0}$. Thus

$$
\sqrt{n}\left(\hat{\gamma}_{n}-\gamma_{0}\right)=-\left(\frac{1}{n} \frac{\partial^{2} Q_{n}\left(\tilde{\gamma}_{n}\right)}{\partial \gamma \partial \gamma^{\prime}}\right)^{-1} \frac{1}{\sqrt{n}} \frac{\partial Q_{n}\left(\gamma_{0}\right)}{\partial \gamma}
$$

Let $\mathbb{W}_{n}=e^{\tau_{0} M_{n}} W_{n} e^{-\tau_{0} M_{n}}$ and $A^{s}=A+A^{\prime}$ for any square matrix $A$. Under some regularity conditions, $\frac{1}{n} \frac{\partial^{2} Q_{n}\left(\tilde{\gamma}_{n}\right)}{\partial \gamma \partial \gamma^{\prime}}=C_{n}+o_{P}(1)$, where

$$
\begin{aligned}
C_{n} & =\mathrm{E}\left(\frac{1}{n} \frac{\partial^{2} Q_{n}\left(\gamma_{0}\right)}{\partial \gamma \partial \gamma^{\prime}}\right) \\
& =\frac{1}{n}\left(\begin{array}{ccc}
\sigma_{0}^{2} \operatorname{tr}\left(\mathbb{W}_{n}^{s} \mathbb{W}_{n}^{s}\right)+2\left(\mathbb{W}_{n} e^{\tau_{0} M_{n}} X_{n} \beta_{0}\right)^{\prime}\left(\mathbb{W}_{n} e^{\tau_{0} M_{n}} X_{n} \beta_{0}\right) & * & * \\
\sigma_{0}^{2} \operatorname{tr}\left(\mathbb{W}_{n}^{s} M_{n}^{s}\right) & \sigma_{0}^{2} \operatorname{tr}\left(M_{n}^{s} M_{n}^{s}\right) & * \\
-2\left(e^{\tau_{0} M_{n}} X_{n}\right)^{\prime} \mathbb{W}_{n} e^{\tau_{0} M_{n}} X_{n} \beta_{0} & 0 & 2\left(e^{\tau_{0} M_{n}} X_{n}\right)^{\prime}\left(e^{\tau_{0} M_{n}} X_{n}\right)
\end{array}\right)
\end{aligned}
$$

As $\operatorname{tr}(A B)=\operatorname{vec}^{\prime}\left(A^{\prime}\right) \operatorname{vec}(B)$ for two conformable matrices $A$ and $B$, where $\operatorname{vec}(\cdot)$ denotes the vectorization of a matrix, $C_{n}$ may be written as $C_{n}=\frac{1}{n} C_{1 n}^{\prime} C_{1 n}$, where

$$
C_{1 n}=\left(\begin{array}{ccc}
\sigma_{0} \operatorname{vec}\left(\mathbb{W}_{n}^{s}\right) & \sigma_{0} \operatorname{vec}\left(M_{n}^{s}\right) & 0 \\
\sqrt{2} \mathbb{W}_{n} e^{\tau_{0} M_{n}} X_{n} \beta_{0} & 0 & -\sqrt{2} e^{\tau_{0} M_{n}} X_{n}
\end{array}\right)
$$

Thus $C_{n}$ is positive semi-definite. The following assumption guarantees that $C_{n}$ is nonsingular in the limit.

Assumption 7. $\lim _{n \rightarrow \infty} \frac{1}{n}\left(\left(\mathbb{W}_{n} e^{\tau_{0} M_{n}} X_{n} \beta_{0}\right)^{\prime} H_{n}\left(\tau_{0}\right)\left(\mathbb{W}_{n} e^{\tau_{0} M_{n}} X_{n} \beta_{0}\right)+\frac{\sigma_{0}^{2}}{2 \operatorname{tr}\left(M_{n}^{s} M_{n}^{s}\right)}\left(\operatorname{tr}\left(\mathbb{W}_{n}^{s} \mathbb{W}_{n}^{s}\right) \operatorname{tr}\left(M_{n}^{s} M_{n}^{s}\right)-\right.\right.$ $\left.\left.\operatorname{tr}^{2}\left(\mathbb{W}_{n}^{s} M_{n}^{s}\right)\right)\right) \neq 0$ and $\lim _{n \rightarrow \infty} \frac{1}{n} \operatorname{tr}\left(M_{n}^{s} M_{n}^{s}\right) \neq 0$.

As $M_{n}^{s} M_{n}^{s}$ in the above assumption is positive semi-definite but not a zero matrix, $\operatorname{tr}\left(M_{n}^{s} M_{n}^{s}\right)>0$. Note that $\left(\mathbb{W}_{n} e^{\tau_{0} M_{n}} X_{n} \beta_{0}\right)^{\prime} H_{n}\left(\tau_{0}\right)\left(\mathbb{W}_{n} e^{\tau_{0} M_{n}} X_{n} \beta_{0}\right) \geq 0$, and $\operatorname{tr}\left(\mathbb{W}_{n}^{s} \mathbb{W}_{n}^{s}\right) \operatorname{tr}\left(M_{n}^{s} M_{n}^{s}\right)-\operatorname{tr}^{2}\left(\mathbb{W}_{n}^{s} M_{n}^{s}\right) \geq 0$ by the Cauchy-Schwarz inequality. The first-order derivatives of $Q_{n}(\gamma)$ at $\gamma_{0}$ are

$$
\begin{aligned}
& \frac{\partial Q_{n}\left(\gamma_{0}\right)}{\partial \alpha}=2\left(\mathbb{W}_{n} e^{\tau_{0} M_{n}} X_{n} \beta_{0}\right)^{\prime} \epsilon_{n}+\epsilon_{n}^{\prime} \mathbb{W}_{n}^{s} \epsilon_{n} \\
& \frac{\partial Q_{n}\left(\gamma_{0}\right)}{\partial \tau}=\epsilon_{n}^{\prime} M_{n}^{s} \epsilon_{n} \\
& \frac{\partial Q_{n}\left(\gamma_{0}\right)}{\partial \beta}=-2\left(e^{\tau_{0} M_{n}} X_{n}\right)^{\prime} \epsilon_{n}
\end{aligned}
$$

which have mean zero and are linear and quadratic functions of $\epsilon_{n}$. Thus the central limit theorem for linear-quadratic forms in Kelejian and Prucha (2001) is applicable. Let $\mu_{3}=\mathrm{E} \epsilon_{n i}^{3}, \mu_{4}=\mathrm{E} \epsilon_{n i}^{4}$, and $\operatorname{vec}_{\mathrm{D}}(A)$ 
be a vector containing the diagonal elements of the square matrix $A$. The VC matrix of $\frac{1}{\sqrt{n}} \frac{\partial Q_{n}\left(\gamma_{0}\right)}{\partial \gamma}$ is

$$
\Omega_{n}=\mathrm{E}\left(\frac{1}{n} \frac{\partial Q_{n}\left(\gamma_{0}\right)}{\partial \gamma} \frac{\partial Q_{n}\left(\gamma_{0}\right)}{\partial \gamma^{\prime}}\right)=2 \sigma_{0}^{2} C_{n}+\Omega_{1 n}
$$

where

$\Omega_{1 n}=\frac{1}{n}\left(\begin{array}{ccc}\left(\mu_{4}-3 \sigma_{0}^{4}\right) \operatorname{vec}_{\mathrm{D}}^{\prime}\left(\mathbb{W}_{n}^{s}\right) \operatorname{vec}_{\mathrm{D}}\left(\mathbb{W}_{n}^{s}\right)+4 \mu_{3}\left(\mathbb{W}_{n} e^{\tau_{0} M_{n}} X_{n} \beta_{0}\right)^{\prime} \operatorname{vec}_{\mathrm{D}}\left(\mathbb{W}_{n}^{s}\right) & 0 & -2 \mu_{3} \operatorname{vec}_{\mathrm{D}}^{\prime}\left(\mathbb{W}_{n}^{s}\right) e^{\tau_{0} M_{n}} X_{n} \\ 0 & 0 & 0 \\ -2 \mu_{3}\left(e^{\tau_{0} M_{n}} X_{n}\right)^{\prime} \operatorname{vec}_{\mathrm{D}}\left(\mathbb{W}_{n}^{s}\right) & 0 & 0\end{array}\right)$

When $\epsilon_{n i}$ 's are normal, $\mu_{3}=\mu_{4}-3 \sigma_{0}^{4}=0$; when $\tau_{0}=0$ or $W_{n}$ and $M_{n}$ are commutative, $\operatorname{vec}_{\mathrm{D}}\left(\mathbb{W}_{n}^{s}\right)=$ $\operatorname{vec}_{\mathrm{D}}\left(W_{n}^{s}\right)=0$ as $W_{n}$ has a zero diagonal. These cases imply that $\Omega_{1 n}=0$ and $\Omega_{n}$ simplifies to $2 \sigma_{0}^{2} C_{n}$. As $\Omega_{n}$ is a VC matrix, it is positive semi-definite. We may also directly show that $\Omega_{n}$ is positive semi-definite. Note that $\mathrm{E}\left(\epsilon_{n i}^{2}-\sigma_{0}^{2}\right)^{2} \mathrm{E} \epsilon_{n i}^{2} \geq\left(\mathrm{E}\left[\left(\epsilon_{n i}^{2}-\sigma_{0}^{2}\right) \epsilon_{n i}\right]\right)^{2}$, i.e. $\left(\mu_{4}-\sigma_{0}^{4}\right) \sigma_{0}^{2} \geq \mu_{3}^{2}$, by the Cauchy-Schwarz inequality. In addition, $\operatorname{tr}\left(\operatorname{Diag}\left(\mathbb{W}_{n}^{s}\right) \operatorname{Diag}\left(\mathbb{W}_{n}^{s}\right)\right)=\operatorname{vec}_{\mathrm{D}}^{\prime}\left(\mathbb{W}_{n}^{s}\right) \operatorname{vec}_{\mathrm{D}}\left(\mathbb{W}_{n}^{s}\right)$, and $\operatorname{tr}\left(\operatorname{Diag}\left(\mathbb{W}_{n}^{s}\right) M_{n}^{s}\right)=0$ as $M_{n}$ has a zero diagonal, where $\operatorname{Diag}(A)$ for a square matrix $A$ denotes a diagonal matrix whose diagonal is equal to that of $A$. Then $\Omega_{n}$ can be written as $\Omega_{n}=\frac{1}{n} \Omega_{2 n}^{\prime} \Omega_{2 n}$, where

$$
\Omega_{2 n}=\left(\begin{array}{ccc}
\sqrt{2} \sigma_{0}^{2} \operatorname{vec}\left(\mathbb{W}_{n}^{s}-\operatorname{Diag}\left(\mathbb{W}_{n}^{s}\right)+\sqrt{\frac{\sigma_{0}^{2}\left(\mu_{4}-\sigma_{0}^{4}\right)-\mu_{3}^{2}}{2 \sigma_{0}^{6}}} \operatorname{Diag}\left(\mathbb{W}_{n}^{s}\right)\right) & \sqrt{2} \sigma_{0}^{2} \operatorname{vec}\left(M_{n}^{s}\right) & 0 \\
2 \sigma_{0} \mathbb{W}_{n} e^{\tau_{0} M_{n}} X_{n} \beta_{0}+\frac{\mu_{3}}{\sigma_{0}} \operatorname{vec}_{\mathrm{D}}\left(\mathbb{W}_{n}^{s}\right) & 0 & -2 \sigma_{0} e^{\tau_{0} M_{n}} X_{n}
\end{array}\right)
$$

Thus $\Omega_{n}$ is positive semi-definite.

Proposition 2. Under Assumptions 1-7, $\sqrt{n}\left(\hat{\gamma}_{n}-\gamma_{0}\right) \stackrel{d}{\rightarrow} N\left(0, \lim _{n \rightarrow \infty} C_{n}^{-1} \Omega_{n} C_{n}^{-1}\right)$. If $\epsilon_{n} \sim N\left(0, \sigma_{0}^{2} I_{n}\right)$; $\tau_{0}=0 ;$ or $W_{n}$ and $M_{n}$ are commutative, then $\sqrt{n}\left(\hat{\gamma}_{n}-\gamma_{0}\right) \stackrel{d}{\rightarrow} N\left(0,2 \sigma_{0}^{2} \lim _{n \rightarrow \infty} C_{n}^{-1}\right)$.

When the disturbances $\epsilon_{n i}$ 's are normal, the generalized information matrix equality holds, thus the limiting distribution of the MLE $\hat{\gamma}_{n}$ does not depend on moments of the disturbances higher than the second order. Even when the disturbances $\epsilon_{n i}$ 's are not normally distributed, if there is no MESS process in the disturbances or the spatial weights matrices $M_{n}$ and $W_{n}$ are commutative, the limiting distribution of the QMLE does not involve moments of the disturbances higher than the second order.

\subsubsection{QMLE: Heteroskedastic Case when $W_{n}$ and $M_{n}$ are Commutative}

When the disturbances $\epsilon_{n i}$ 's are independent but may have different variances, the following assumption is made about the disturbances. 
Assumption 8. The $\epsilon_{n i}$ 's are independent $\left(0, \sigma_{n i}^{2}\right)$ and the moments $\mathrm{E}\left|\epsilon_{n i}\right|^{4+\eta}$ for some $\eta>0$ exist and are uniformly bounded for all $n$ and $i$.

As argued earlier, when $W_{n}$ and $M_{n}$ can commute, or $\tau_{0}=0$, the minimization of the function $Q_{n}(\gamma)$ may yield a consistent estimator $\hat{\gamma}_{n}$ of $\gamma$ under unknown heteroskedasticity, since the first-order derivatives of $Q_{n}(\gamma)$ at $\gamma_{0}$ have zero expectation. In practice for some situations, one may use a single spatial weights matrix $W_{n}$ for both the main equation and the disturbance process. This case implies the commutative property.

Assumption 9. $W_{n}$ and $M_{n}$ are commutative or $\tau_{0}=0$.

Define $\bar{Q}_{n}(\phi)=\min _{\beta} \mathrm{E} Q_{n}(\gamma)$. The identification of $\phi_{0}$ can be based on minimizers of $\left\{\bar{Q}_{n}(\phi)\right\}$. Using Assumption 9, we have $\bar{Q}_{n}(\phi)=\bar{Q}_{1 n}(\phi)+\bar{Q}_{2 n}(\phi)$, where

$$
\begin{aligned}
& \bar{Q}_{1 n}(\phi)=\left(X_{n} \beta_{0}\right)^{\prime} e^{\left(\alpha-\alpha_{0}\right) W_{n}^{\prime}} e^{\tau M_{n}^{\prime}} H_{n}(\tau) e^{\tau M_{n}} e^{\left(\alpha-\alpha_{0}\right) W_{n}} X_{n} \beta_{0}, \\
& \bar{Q}_{2 n}(\phi)=\operatorname{tr}\left(e^{\left(\alpha-\alpha_{0}\right) W_{n}^{\prime}} e^{\left(\tau-\tau_{0}\right) M_{n}^{\prime}} e^{\left(\tau-\tau_{0}\right) M_{n}} e^{\left(\alpha-\alpha_{0}\right) W_{n}} \Sigma_{n}\right) .
\end{aligned}
$$

It is obvious that $\bar{Q}_{1 n}(\phi) \geq 0$ and $\bar{Q}_{1 n}\left(\phi_{0}\right)=0$. As $W_{n}$ and $M_{n}$ have zero diagonals and $\Sigma_{n}$ is a diagonal matrix, $\frac{\partial \bar{Q}_{2 n}\left(\phi_{0}\right)}{\partial \alpha}=\operatorname{tr}\left[\left(W_{n}^{\prime}+W_{n}\right) \Sigma_{n}\right]=0$ and $\frac{\partial \bar{Q}_{2 n}\left(\phi_{0}\right)}{\partial \tau}=\operatorname{tr}\left[\left(M_{n}^{\prime}+M_{n}\right) \Sigma_{n}\right]=0$. Thus $\phi_{0}$ is a stationary point of $\bar{Q}_{2 n}(\phi)$ and also $\bar{Q}_{n}(\phi)$. Using the commutative property of $W_{n}$ and $M_{n}$, we have $\frac{\partial^{2} \bar{Q}_{2 n}(\phi)}{\partial \alpha^{2}}=$ $\operatorname{tr}\left[\Sigma_{n}^{1 / 2} e^{\left(\alpha-\alpha_{0}\right) W_{n}^{\prime}} e^{\left(\tau-\tau_{0}\right) M_{n}^{\prime}}\left(W_{n}^{\prime 2}+W_{n}^{2}+2 W_{n}^{\prime} W_{n}\right) e^{\left(\tau-\tau_{0}\right) M_{n}} e^{\left(\alpha-\alpha_{0}\right) W_{n} \Sigma_{n}^{1 / 2}}\right]$,

$$
\frac{\partial^{2} \bar{Q}_{2 n}(\phi)}{\partial \tau^{2}}=\operatorname{tr}\left[\Sigma_{n}^{1 / 2} e^{\left(\alpha-\alpha_{0}\right) W_{n}^{\prime}} e^{\left(\tau-\tau_{0}\right) M_{n}^{\prime}}\left(M_{n}^{\prime 2}+M_{n}^{2}+2 M_{n}^{\prime} M_{n}\right) e^{\left(\tau-\tau_{0}\right) M_{n}} e^{\left(\alpha-\alpha_{0}\right) W_{n}} \Sigma_{n}^{1 / 2}\right]
$$

and

$$
\begin{aligned}
\frac{\partial^{2} \bar{Q}_{2 n}(\phi)}{\partial \alpha \partial \tau} & =2 \operatorname{tr}\left\{\Sigma_{n}^{1 / 2} e^{\left(\alpha-\alpha_{0}\right) W_{n}^{\prime}} e^{\left(\tau-\tau_{0}\right) M_{n}^{\prime}}\left[\left(W_{n}^{\prime}+W_{n}\right) M_{n}\right] e^{\left(\tau-\tau_{0}\right) M_{n}} e^{\left.\left(\alpha-\alpha_{0}\right) W_{n} \Sigma_{n}^{1 / 2}\right\}}\right. \\
& =\operatorname{tr}\left\{\Sigma_{n}^{1 / 2} e^{\left(\alpha-\alpha_{0}\right) W_{n}^{\prime}} e^{\left(\tau-\tau_{0}\right) M_{n}^{\prime}}\left[\left(W_{n}^{\prime}+W_{n}\right) M_{n}+M_{n}^{\prime} W_{n}+W_{n}^{\prime} M_{n}^{\prime}\right] e^{\left(\tau-\tau_{0}\right) M_{n}} e^{\left(\alpha-\alpha_{0}\right) W_{n}} \Sigma_{n}^{1 / 2}\right\}
\end{aligned}
$$

If $W_{n}^{\prime} W_{n}=W_{n} W_{n}^{\prime}$, then

$\frac{\partial^{2} \bar{Q}_{2 n}(\phi)}{\partial \alpha^{2}}=\operatorname{tr}\left[\Sigma_{n}^{1 / 2} e^{\left(\alpha-\alpha_{0}\right) W_{n}^{\prime}} e^{\left(\tau-\tau_{0}\right) M_{n}^{\prime}}\left(W_{n}^{\prime}+W_{n}\right)^{2} e^{\left(\tau-\tau_{0}\right) M_{n}} e^{\left.\left(\alpha-\alpha_{0}\right) W_{n} \Sigma_{n}^{1 / 2}\right]} \geq 0\right.$; if $M_{n}^{\prime} M_{n}=M_{n} M_{n}^{\prime}$, then

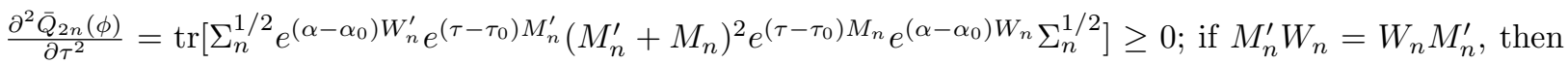
$\frac{\partial^{2} \bar{Q}_{2 n}(\phi)}{\partial \alpha \partial \tau}=\operatorname{tr}\left\{\Sigma_{n}^{1 / 2} e^{\left(\alpha-\alpha_{0}\right) W_{n}^{\prime}} e^{\left(\tau-\tau_{0}\right) M_{n}^{\prime}}\left(W_{n}^{\prime}+W_{n}\right)\left(M_{n}^{\prime}+M_{n}\right) e^{\left(\tau-\tau_{0}\right) M_{n}} e^{\left.\left(\alpha-\alpha_{0}\right) W_{n} \Sigma_{n}^{1 / 2}\right\}}\right.$. Thus, under the conditions that $W_{n}^{\prime} W_{n}=W_{n} W_{n}^{\prime}, M_{n}^{\prime} M_{n}=M_{n} M_{n}^{\prime}$ and $M_{n}^{\prime} W_{n}=W_{n} M_{n}^{\prime}$, by the Cauchy-Schwarz inequality, $\frac{\partial^{2} \bar{Q}_{2 n}(\phi)}{\partial \alpha^{2}} \frac{\partial^{2} \bar{Q}_{2 n}(\phi)}{\partial \tau^{2}} \geq\left(\frac{\partial^{2} \bar{Q}_{2 n}(\phi)}{\partial \alpha \partial \tau}\right)^{2}$. In this case, $\frac{\partial^{2} \bar{Q}_{2 n}(\phi)}{\partial \phi \partial \phi^{\prime}}$ is positive semi-definite and $\bar{Q}_{2 n}(\phi)$ is a concave 
function. It follows that $\phi_{0}$ is a global minimizer of $\bar{Q}_{2 n}(\phi)$ and $\bar{Q}_{n}(\phi)$. Hence, with some extra conditions on $W_{n}$ and $M_{n}$, it is possible that $\phi_{0}$ uniquely minimizes $\bar{Q}_{n}(\phi) / n$ in the limit.

It is also possible that $\phi_{0}$ is only a local minimizer of $\bar{Q}_{n}(\phi)$. For example, in the case that $W_{n}=M_{n}$, $\frac{\partial^{2} \bar{Q}_{2 n}\left(\phi_{0}\right)}{\partial \alpha^{2}}=\frac{\partial^{2} \bar{Q}_{2 n}\left(\phi_{0}\right)}{\partial \tau^{2}}=\frac{\partial^{2} \bar{Q}_{2 n}\left(\phi_{0}\right)}{\partial \alpha \partial \tau}=\operatorname{tr}\left[\left(W_{n}^{\prime 2}+W_{n}^{2}+2 W_{n}^{\prime} W_{n}\right) \Sigma_{n}\right]$, which is positive if elements of $W_{n}$ are non-negative. Then $\frac{\partial^{2} \bar{Q}_{2 n}\left(\phi_{0}\right)}{\partial \phi \partial \phi^{\prime}}$ is positive semi-definite and $\bar{Q}_{2 n}(\phi)$ is concave at $\phi_{0}$. Hence, $\phi_{0}$ is a local minimizer of $\bar{Q}_{2 n}(\phi)$ and $\bar{Q}_{n}(\phi)$. These considerations motivate the following identification condition.

Assumption 10. $\lim _{n \rightarrow \infty} \frac{1}{n}\left[\bar{Q}_{n}(\phi)-\operatorname{tr}\left(\Sigma_{n}\right)\right]>0$ for any $\phi \neq \phi_{0}$.

Proposition 3. Under Assumptions 1-4 and 8-10, the QMLE $\hat{\gamma}_{n}$ is consistent for $\gamma_{0}$.

Let

$$
\begin{aligned}
D_{n} & =\frac{1}{n} \mathrm{E}\left(\frac{\partial^{2} Q_{n}\left(\gamma_{0}\right)}{\partial \gamma \partial \gamma^{\prime}}\right) \\
& =\frac{2}{n}\left(\begin{array}{ccc}
\operatorname{tr}\left(W_{n}^{s} W_{n} \Sigma_{n}\right)+\left(W_{n} e^{\tau_{0} M_{n}} X_{n} \beta_{0}\right)^{\prime}\left(W_{n} e^{\tau_{0} M_{n}} X_{n} \beta_{0}\right) & * & * \\
\operatorname{tr}\left(M_{n}^{s} W_{n} \Sigma_{n}\right) & \operatorname{tr}\left(M_{n}^{s} M_{n} \Sigma_{n}\right) & * \\
-\left(e^{\tau_{0} M_{n}} X_{n}\right)^{\prime} W_{n} e^{\tau_{0} M_{n}} X_{n} \beta_{0} & 0 & \left(e^{\tau_{0} M_{n}} X_{n}\right)^{\prime}\left(e^{\tau_{0} M_{n}} X_{n}\right)
\end{array}\right),
\end{aligned}
$$

and

$$
\begin{aligned}
\Delta_{n}= & \frac{1}{n} \mathrm{E}\left(\frac{\partial Q_{n}\left(\gamma_{0}\right)}{\partial \gamma} \frac{\partial Q_{n}\left(\gamma_{0}\right)}{\partial \gamma^{\prime}}\right) \\
& =\frac{2}{n}\left(\begin{array}{ccc}
\operatorname{tr}\left(\Sigma_{n} W_{n}^{s} \Sigma_{n} W_{n}^{s}\right)+2\left(W_{n} e^{\tau_{0} M_{n}} X_{n} \beta_{0}\right)^{\prime} \Sigma_{n}\left(W_{n} e^{\tau_{0} M_{n}} X_{n} \beta_{0}\right) & * & * \\
\operatorname{tr}\left(\Sigma_{n} M_{n}^{s} \Sigma_{n} W_{n}^{s}\right) & \operatorname{tr}\left(\Sigma_{n} M_{n}^{s} \Sigma_{n} M_{n}^{s}\right) & * \\
-2\left(e^{\tau_{0} M_{n}} X_{n}\right)^{\prime} \Sigma_{n} W_{n} e^{\tau_{0} M_{n}} X_{n} \beta_{0} & 0 & 2\left(e^{\tau_{0} M_{n}} X_{n}\right)^{\prime} \Sigma_{n}\left(e^{\tau_{0} M_{n}} X_{n}\right)
\end{array}\right) .
\end{aligned}
$$

Note that $\Delta_{n}$, being the VC matrix of a vector of linear-quadratic forms of disturbances, does not involve higher than the second moments of disturbances, because $W_{n}$ and $M_{n}$ in the quadratic forms $\epsilon_{n}^{\prime} W_{n}^{s} \epsilon_{n}$ and $\epsilon_{n}^{\prime} M_{n}^{s} \epsilon_{n}$ have zero diagonals (see Lee, 2007). We may write $\Delta_{n}$ as $\Delta_{n}=\frac{1}{n} \Delta_{1 n}^{\prime} \Delta_{1 n}$, where

$$
\Delta_{1 n}=\left(\begin{array}{ccc}
\sqrt{2} \operatorname{vec}\left(\Sigma_{n}^{1 / 2} W_{n}^{s} \Sigma_{n}^{1 / 2}\right) & \sqrt{2} \operatorname{vec}\left(\Sigma_{n}^{1 / 2} M_{n}^{s} \Sigma_{n}^{1 / 2}\right) & 0 \\
2 \Sigma_{n}^{1 / 2} W_{n} e^{\tau_{0} M_{n}} X_{n} \beta_{0} & 0 & -2 \Sigma_{n}^{1 / 2} e^{\tau_{0} M_{n}} X_{n}
\end{array}\right)
$$

thus $\Delta_{n}$ is positive semi-definite. To make sure that $D_{n}$ is invertible for large enough $n$, we need the following assumption. 
Assumption 11. $\lim _{n \rightarrow \infty} \frac{1}{n} \operatorname{tr}\left(M_{n}^{s} M_{n} \Sigma_{n}\right) \neq 0$ and $\lim _{n \rightarrow \infty} \frac{1}{n}\left(\left(W_{n} e^{\tau_{0} M_{n}} X_{n} \beta_{0}\right)^{\prime} H_{n}\left(\tau_{0}\right)\left(W_{n} e^{\tau_{0} M_{n}} X_{n} \beta_{0}\right)+\right.$ $\left.\frac{\operatorname{tr}\left(W_{n}^{s} W_{n} \Sigma_{n}\right) \operatorname{tr}\left(M_{n}^{s} M_{n} \Sigma_{n}\right)-\operatorname{tr}^{2}\left(M_{n}^{s} W_{n} \Sigma_{n}\right)}{\operatorname{tr}\left(M_{n}^{s} M_{n} \Sigma_{n}\right)}\right) \neq 0$.

When elements of $W_{n}$ and $M_{n}$ are non-negative, $\operatorname{tr}\left(M_{n}^{s} M_{n} \Sigma_{n}\right)>0, \operatorname{tr}\left(M_{n}^{s} W_{n} \Sigma_{n}\right) \geq 0$ and $\operatorname{tr}\left(W_{n}^{s} W_{n} \Sigma_{n}\right)>$ 0 , because $M_{n}$ and $W_{n}$ are not zero matrices and the diagonal elements of $\Sigma_{n}$ are positive in general.

Proposition 4. Under Assumptions $1-4$ and $8-11, \sqrt{n}\left(\hat{\gamma}_{n}-\gamma_{0}\right) \stackrel{d}{\rightarrow} N\left(0, \lim _{n \rightarrow \infty} D_{n}^{-1} \Delta_{n} D_{n}^{-1}\right)$.

With the requirement of $\tau_{0}=0$ or commutativeness of $W_{n}$ and $M_{n}$, in addition to the consistency, the QMLE under unknown heteroskedasticity has an asymptotic distribution that does not involve higher than the second moments of the disturbances, whether the disturbances are normal or not.

To make asymptotically valid inference using the QMLE $\hat{\gamma}_{n}$ under unknown heteroskedasticity, we need a consistent estimator for $D_{n}^{-1} \Delta_{n} D_{n}^{-1}$. As in White (1980), we may have a consistent estimator of $D_{n}^{-1} \Delta_{n} D_{n}^{-1}$ without being able to consistently estimate $\Sigma_{n}$, which has $n$ unknown parameters. Let $\hat{\Sigma}_{n}=\operatorname{Diag}\left(\hat{\epsilon}_{n 1}^{2}, \ldots, \hat{\epsilon}_{n n}^{2}\right)$, where $\hat{\epsilon}_{n}=\left(\hat{\epsilon}_{n 1}, \ldots, \hat{\epsilon}_{n n}\right)^{\prime}$ is the residual vector from the QML estimation. Consistent estimators for $D_{n}$ and $\Delta_{n}$ can be, respectively, $\hat{D}_{n}$ and $\hat{\Delta}_{n}$, which are the matrices derived from replacing $\Sigma_{n}$ in $D_{n}$ and $\Omega_{n}$ by $\hat{\Sigma}_{n}$, and replacing $\gamma_{0}$ by a consistent estimator $\hat{\gamma}_{n}$. The $\hat{D}_{n}$ and $\hat{\Delta}_{n}$ can be consistent because $D_{n}$ and $\Delta_{n}$ with fixed dimensions are estimated as whole terms.

Proposition 5. Under Assumptions 1-4 and 8-10, $\hat{D}_{n}=D_{n}+o_{P}(1)$ and $\hat{\Delta}_{n}=\Delta_{n}+o_{P}(1)$.

\section{2. $G M M E$}

We now consider the GMM estimation of the $\operatorname{MESS}(1,1)$. Let the moment vector be

$$
g_{n}(\gamma)=\frac{1}{n}\left(\epsilon_{n}^{\prime}(\gamma) P_{n 1} \epsilon_{n}(\gamma), \ldots, \epsilon_{n}^{\prime}(\gamma) P_{n, k_{p}} \epsilon_{n}(\gamma), \epsilon_{n}^{\prime}(\gamma) F_{n}\right)^{\prime}
$$

where $\epsilon_{n}(\gamma)=e^{\tau M_{n}}\left(e^{\alpha W_{n}} y_{n}-X_{n} \beta\right)$, the $n$-dimensional square matrices $P_{n i}$ 's for the quadratic moments have zero traces when $\epsilon_{n i}$ 's are i.i.d. and have zero diagonals when $\epsilon_{n i}$ 's are independent but with different variances, and the $n \times k_{f}$ instrumental variable matrix $F_{n}$ used in the 2SLS approach can consist of the independent columns of $X_{n}, W_{n} X_{n}, M_{n} X_{n}, W_{n}^{2} X_{n}, M_{n}^{2} X_{n}$ and so on. ${ }^{15}$ The GMM objective function with the weighting matrix $a_{n} a_{n}^{\prime}$ is $g_{n}^{\prime}(\gamma) a_{n} a_{n}^{\prime} g_{n}(\gamma)$, where the full column rank $\left(k_{p}+k_{f}\right) \times k_{a}$ matrix $a_{n}$ with $k_{a} \geq k+2$ converges to a full rank matrix $a_{0}$ by design.

\footnotetext{
${ }^{15}$ For $\alpha$ and $\beta$, we may use only the linear instrument $F_{n}$ and implement a 2SLS estimation, for which the objective function

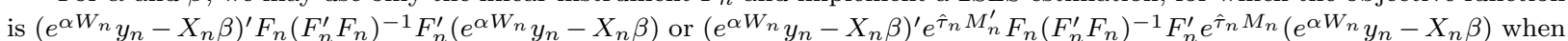
taking into account the MESS process in the disturbances, where $\hat{\tau}_{n}$ is an initial consistent estimator of $\tau$. This is a nonlinear 2SLS that does not have a closed-form solution. Thus it does not have a computational advantage as the traditional 2 SLS and we do not discuss it separately.
} 


\subsubsection{GMME: Homoskedastic Case}

When the disturbances are i.i.d., the GMME can be consistent when the matrices $P_{n i}$ 's have zero traces but not necessarily zero diagonals. The $P_{n i}$ 's are constructed from $W_{n}$ and $M_{n}$, thus we may assume that $P_{n i}$ 's are bounded in row and column sum norms.

Assumption 12. The $n$-dimensional square matrices $P_{n 1}, \ldots, P_{n, k_{p}}$ have zero traces and are bounded in both row and column sum norms. Elements of $F_{n}$ are uniformly bounded constants.

For any $\gamma$,

$$
\begin{aligned}
\mathrm{E}\left[\epsilon_{n}^{\prime}(\gamma) P_{n i} \epsilon_{n}(\gamma)\right]= & \left(e^{\left(\alpha-\alpha_{0}\right) W_{n}} X_{n} \beta_{0}-X_{n} \beta\right)^{\prime} e^{\tau M_{n}^{\prime}} P_{n i} e^{\tau M_{n}}\left(e^{\left(\alpha-\alpha_{0}\right) W_{n}} X_{n} \beta_{0}-X_{n} \beta\right) \\
& +\sigma_{0}^{2} \operatorname{tr}\left(e^{-\tau_{0} M_{n}^{\prime}} e^{\left(\alpha-\alpha_{0}\right) W_{n}^{\prime}} e^{\tau M_{n}^{\prime}} P_{n i} e^{\tau M_{n}} e^{\left(\alpha-\alpha_{0}\right) W_{n}} e^{-\tau_{0} M_{n}}\right), \\
\mathrm{E}\left[F_{n}^{\prime} \epsilon_{n}(\gamma)\right]= & F_{n}^{\prime} e^{\tau M_{n}}\left(e^{\left(\alpha-\alpha_{0}\right) W_{n}} X_{n} \beta_{0}-X_{n} \beta\right) .
\end{aligned}
$$

The identification of $\gamma_{0}$ requires the unique solution of the limiting equations $\lim _{n \rightarrow \infty} \operatorname{E~} g_{n}(\gamma)=0$ at $\gamma_{0}$. When $\alpha=\alpha_{0}$ and $\beta=\beta_{0}, \mathrm{E}\left[F_{n}^{\prime} \epsilon_{n}(\gamma)\right]=0$ whatever $\tau$ is. Thus $\tau$ cannot be identified from the linear moments $\mathrm{E}\left[F_{n}^{\prime} \epsilon_{n}(\gamma)\right]=0$, because it only plays a role as weighting. It is possible that $\alpha_{0}$ and $\beta_{0}$ may be identified from $\mathrm{E}\left[F_{n}^{\prime} \epsilon_{n}(\gamma)\right]=0$, and $\tau_{0}$ be identified from the quadratic moments $\mathrm{E}\left[\epsilon_{n}^{\prime}(\gamma) P_{n i} \epsilon_{n}(\gamma)\right]=0$, $i=1, \ldots, k_{p}$. Let $F_{n}=\left(F_{1 n}, F_{2 n}\right)$ such that $\lim _{n \rightarrow \infty} \frac{1}{n} F_{2 n}^{\prime} e^{\tau M_{n}} X_{n}$ is nonsingular for any $\tau \in[-\delta, \delta]$, which is a part of a rank condition for valid IV's. The $\mathrm{E}\left[F_{n}^{\prime} \epsilon_{n}(\gamma)\right]=0$ is equivalent to

$$
\begin{aligned}
& F_{1 n}^{\prime} e^{\tau M_{n}}\left(e^{\left(\alpha-\alpha_{0}\right) W_{n}} X_{n} \beta_{0}-X_{n} \beta\right)=0, \\
& F_{2 n}^{\prime} e^{\tau M_{n}}\left(e^{\left(\alpha-\alpha_{0}\right) W_{n}} X_{n} \beta_{0}-X_{n} \beta\right)=0 .
\end{aligned}
$$

From (29), we have $\beta=\left(F_{2 n}^{\prime} e^{\tau M_{n}} X_{n}\right)^{-1} F_{2 n}^{\prime} e^{\tau M_{n}} e^{\left(\alpha-\alpha_{0}\right) W_{n}} X_{n} \beta_{0}$. With substitution, (28) becomes

$$
F_{1 n}^{\prime} H_{1 n}(\tau) e^{\tau M_{n}} e^{\left(\alpha-\alpha_{0}\right) W_{n}} X_{n} \beta_{0}=0
$$

where $H_{1 n}(\tau)=I_{n}-e^{\tau M_{n}} X_{n}\left(F_{2 n}^{\prime} e^{\tau M_{n}} X_{n}\right)^{-1} F_{2 n}^{\prime}$. It is apparent that when $\alpha=\alpha_{0}, F_{1 n}^{\prime} H_{1 n}(\tau) e^{\tau M_{n}} X_{n} \beta_{0}=$ 0 . So we just want to rule out the possibility that $F_{1 n}^{\prime} H_{1 n}(\tau) e^{\tau M_{n}} e^{\eta W_{n}} X_{n} \beta_{0}=0$ for some $\eta \neq 0$. When $\alpha=\alpha_{0}$ and $\beta=\beta_{0},(26)$ becomes $\sigma_{0}^{2} \operatorname{tr}\left(e^{\left(\tau-\tau_{0}\right) M_{n}^{\prime}} P_{n i} e^{\left(\tau-\tau_{0}\right) M_{n}}\right)=0$. Then for the identification of $\tau_{0}$, it requires some matrix $P_{n i}$ such that $\lim _{n \rightarrow \infty} \frac{1}{n} \operatorname{tr}\left(e^{\left(\tau-\tau_{0}\right) M_{n}^{\prime}} P_{n i} e^{\left(\tau-\tau_{0}\right) M_{n}}\right) \neq 0$ when $\tau \neq \tau_{0}$. We thus make the following identification assumption. 
Assumption 13. $\lim _{n \rightarrow \infty} \frac{1}{n}\left[\operatorname{tr}\left(e^{\left(\tau-\tau_{0}\right) M_{n}^{\prime}} P_{n 1} e^{\left(\tau-\tau_{0}\right) M_{n}}\right), \ldots, \operatorname{tr}\left(e^{\left(\tau-\tau_{0}\right) M_{n}^{\prime}} P_{n, k_{p}} e^{\left(\tau-\tau_{0}\right) M_{n}}\right)\right] \neq 0$ for any $\tau \neq$ $\tau_{0}$, and $F_{n}$ may be written as $F_{n}=\left(F_{1 n}, F_{2 n}\right)$ such that $\lim _{n \rightarrow \infty} \frac{1}{n} F_{2 n}^{\prime} e^{\tau M_{n}} X_{n}$ is nonsingular for any $\tau \in[-\delta, \delta]$, and $\lim _{n \rightarrow \infty} \frac{1}{n} F_{1 n}^{\prime} H_{1 n}(\tau) e^{\tau M_{n}} e^{\eta W_{n}} X_{n} \beta_{0} \neq 0$ for any $\eta \neq 0$ and $\tau \in[-\delta, \delta]$.

As usual for nonlinear extremum estimators, we assume the compactness of the parameter space of $\gamma$ (Amemiya, 1985).

Assumption 14. The parameter space $\Gamma$ of $\gamma$ is compact and the true $\gamma_{0}$ is in the interior of $\Gamma$.

Proposition 6. Under Assumptions 1, 2, 5 and 12-14, the GMM estimator $\hat{\gamma}_{n}$ from the minimization of $g_{n}^{\prime}(\gamma) a_{n} a_{n}^{\prime} g_{n}(\gamma)$ is a consistent estimator of $\gamma_{0}$, and

$$
\sqrt{n}\left(\hat{\gamma}_{n}-\gamma_{0}\right) \stackrel{d}{\rightarrow} N\left(0, \lim _{n \rightarrow \infty}\left(G_{n}^{\prime} a_{n} a_{n}^{\prime} G_{n}\right)^{-1} G_{n}^{\prime} a_{n} a_{n}^{\prime} V_{n} a_{n} a_{n}^{\prime} G_{n}\left(G_{n}^{\prime} a_{n} a_{n}^{\prime} G_{n}\right)^{-1}\right)
$$

where

$$
V_{n}=n \mathrm{E}\left[g_{n}\left(\gamma_{0}\right) g_{n}^{\prime}\left(\gamma_{0}\right)\right]=\frac{1}{n}\left(\begin{array}{cc}
\frac{\sigma_{0}^{4}}{2} \omega_{n}^{\prime} \omega_{n}+\frac{1}{4}\left(\mu_{4}-3 \sigma_{0}^{4}\right) \omega_{n d}^{\prime} \omega_{n d} & \frac{1}{2} \mu_{3} \omega_{n d}^{\prime} F_{n} \\
\frac{1}{2} \mu_{3} F_{n}^{\prime} \omega_{n d} & \sigma_{0}^{2} F_{n}^{\prime} F_{n}
\end{array}\right)
$$

and

$$
G_{n}=\mathrm{E} \frac{\partial g_{n}\left(\gamma_{0}\right)}{\partial \gamma^{\prime}}=\frac{1}{n}\left(\begin{array}{ccc}
\frac{\sigma_{0}^{2}}{2} \omega_{n}^{\prime} \operatorname{vec}\left(\mathbb{W}_{n}^{s}\right) & \frac{\sigma_{0}^{2}}{2} \omega_{n}^{\prime} \operatorname{vec}\left(M_{n}^{s}\right) & 0 \\
F_{n}^{\prime} \mathbb{W}_{n} e^{\tau_{0} M_{n}} X_{n} \beta_{0} & 0 & -F_{n}^{\prime} e^{\tau_{0} M_{n}} X_{n}
\end{array}\right)
$$

with $\omega_{n}=\left(\operatorname{vec}\left(P_{n 1}^{s}\right), \ldots, \operatorname{vec}\left(P_{n, k_{p}}^{s}\right)\right)$ and $\omega_{n d}=\left(\operatorname{vec}_{\mathrm{D}}\left(P_{n 1}^{s}\right), \ldots, \operatorname{vec}_{\mathrm{D}}\left(P_{n, k_{p}}^{s}\right)\right)$, under the condition that $\lim _{n \rightarrow \infty} a_{n}^{\prime} G_{n}$ exists and has the full rank $k+2$.

Within the GMM framework, with moments $g_{n}(\gamma)$, an optimum GMM will use $V_{n}^{-1}$ as the optimum weighting in place of $a_{n} a_{n}^{\prime}$. The variance matrix $V_{n}$ of $g_{n}\left(\gamma_{0}\right)$ in the preceding proposition can be put into a more informative form as a positive semi-definite matrix. Let $\omega_{n}^{\#}=\left(\operatorname{vec}\left(P_{n 1}^{\# s}\right), \ldots, \operatorname{vec}\left(P_{n, k_{p}}^{\# s}\right)\right)$, where $P_{n i}^{\# s}=\frac{1}{2} \sqrt{\mu_{4}-\sigma_{0}^{4}-\frac{\mu_{3}^{2}}{\sigma_{0}^{2}}} \operatorname{Diag}\left(P_{n i}^{s}\right)+\frac{\sqrt{2} \sigma_{0}^{2}}{2}\left[P_{n i}^{s}-\operatorname{Diag}\left(P_{n i}^{s}\right)\right]$, then

$$
V_{n}=\frac{1}{n}\left(\begin{array}{cc}
\omega_{n}^{\#} & 0 \\
\frac{\mu_{3}}{2 \sigma_{0}} \omega_{n d} & \sigma_{0} F_{n}
\end{array}\right)^{\prime}\left(\begin{array}{cc}
\omega_{n}^{\#} & 0 \\
\frac{\mu_{3}}{2 \sigma_{0}} \omega_{n d} & \sigma_{0} F_{n}
\end{array}\right)
$$

Thus $V_{n}$ is positive semi-definite. We require the non-singularity of $V_{n}$ to formulate the feasible optimal GMM, which is guaranteed by the following assumption.

Assumption 15. The limits of $\frac{1}{n} F_{n}^{\prime} F_{n}$ and $\frac{\sigma_{0}^{4}}{2 n}\left(\omega_{n}^{\prime} \omega_{n}-\omega_{n d}^{\prime} \omega_{n d}\right)+\frac{1}{4 n}\left(\mu_{4}-\sigma_{0}^{2}-\frac{\mu_{3}^{2}}{\sigma_{0}^{2}}\right) \omega_{n d}^{\prime} \omega_{n d}+\frac{\mu_{3}^{2}}{4 n \sigma_{0}^{2}} \omega_{n d}^{\prime} H_{F_{n}} \omega_{n d}$ exist and are nonsingular, where $H_{F_{n}}=I_{n}-F_{n}\left(F_{n}^{\prime} F_{n}\right)^{-1} F_{n}^{\prime}$. 
Note that $\omega_{n}^{\prime} \omega_{n}-\omega_{n d}^{\prime} \omega_{n d}=\left(\operatorname{vec}\left(P_{n 1}^{\# s}-\operatorname{Diag}\left(P_{n 1}^{\# s}\right)\right), \ldots, \operatorname{vec}\left(P_{n, k_{p}}^{\# s}-\operatorname{Diag}\left(P_{n, k_{p}}^{\# s}\right)\right)\right)^{\prime}\left(\operatorname{vec}\left(P_{n 1}^{\# s}-\operatorname{Diag}\left(P_{n 1}^{\# s}\right)\right)\right.$, $\ldots, \operatorname{vec}\left(P_{n, k_{p}}^{\# s}-\operatorname{Diag}\left(P_{n, k_{p}}^{\# s}\right)\right) \geq 0$. When $\lim _{n \rightarrow \infty} \frac{1}{n} F_{n}^{\prime} F_{n}$ is nonsingular, the above assumption is satisfied as long as one of the terms $\lim _{n \rightarrow \infty} \frac{1}{n}\left(\omega_{n}^{\prime} \omega_{n}-\omega_{n d}^{\prime} \omega_{n d}\right), \lim _{n \rightarrow \infty} \frac{1}{n} \omega_{n d}^{\prime} \omega_{n d}$, and $\lim _{n \rightarrow \infty} \frac{\mu_{3}^{2}}{n} \omega_{n d}^{\prime} H_{F_{n}} \omega_{n d}$ is nonsingular. A consistent estimator $\hat{V}_{n}$ for $V_{n}$ may be obtained from replacing the $\sigma_{0}^{2}, \mu_{3}$ and $\mu_{4}$ in $V_{n}$ by their consistent estimators.

Proposition 7. Under Assumptions 1, 2, 5 and 12-15, the feasible optimal GMME $\hat{\gamma}_{n, o}$ from the minimization of $g_{n}^{\prime}(\gamma) \hat{V}_{n}^{-1} g_{n}(\gamma)$ is a consistent estimator of $\gamma_{0}$, and

$$
\sqrt{n}\left(\hat{\gamma}_{n, o}-\gamma_{0}\right) \stackrel{d}{\rightarrow} N\left(0, \lim _{n \rightarrow \infty}\left(G_{n}^{\prime} V_{n}^{-1} G_{n}\right)^{-1}\right)
$$

As the selections of linear and quadratic moments via $F_{n}$ and $P_{n i}$ 's are many, there is an issue on the best design on those matrices. We shall show the existence of a best GMME within the class of GMMEs with linear and quadratic moments, in the sense that it has the smallest VC matrix. For that purpose, we follow Breusch et al. (1999) to show that additional linear and quadratic moments are redundant given properly selected ones. ${ }^{16}$ If $e^{\tau_{0} M_{n}} X_{n}$ contains an intercept term due to the presence of an intercept term in $X_{n}$, let $X_{n}^{*}$ be the submatrix of $X_{n}$ with the intercept term deleted, so that $e^{\tau_{0} M_{n}} X_{n}=\left[e^{\tau_{0} M_{n}} X_{n}^{*}, c\left(\tau_{0}\right) l_{n}\right]$, where $c\left(\tau_{0}\right)$ is a scalar function of $\tau_{0} \cdot{ }^{17}$ Otherwise, $X_{n}^{*}=X_{n}$ and $e^{\tau_{0} M_{n}} X_{n}^{*}=e^{\tau_{0} M_{n}} X_{n}$. Suppose that there are $k^{*}$ columns in $X_{n}^{*}$. Let $X_{n l}^{*}$ be the $l$ th column of $X_{n}^{*}, \eta_{3}=\mu_{3} \sigma_{0}^{-3}$ and $\eta_{4}=\mu_{4} \sigma_{0}^{-4}$ be the skewness and kurtosis of the disturbances. Furthermore, let $A_{n}^{(t)}=A_{n}-I_{n} \operatorname{tr}\left(A_{n}\right) / n$ for any $n \times n$ matrix $A_{n}$, which is the matrix $A_{n}$ with its trace subtracted out from is diagonal. Thus $A_{n}^{(t)}$ has zero trace.

Proposition 8. Suppose that Assumptions 1, 2, 5, and 12-15 hold. Let

$$
g_{n}^{*}(\gamma)=\frac{1}{n}\left(P_{n 1}^{*} \epsilon(\gamma), \ldots, P_{n, k^{*}+4}^{*} \epsilon(\gamma), F_{n}^{*}\right)^{\prime} \epsilon(\gamma)
$$

where $P_{n 1}^{*}=\mathbb{W}_{n}, P_{n 2}^{*}=\operatorname{Diag}\left(\mathbb{W}_{n}\right), P_{n 3}^{*}=\operatorname{Diag}\left(e^{\tau_{0} M_{n}} W_{n} X_{n} \beta_{0}\right)^{(t)}, P_{n 4}^{*}=M_{n}, P_{n, l+4}^{*}=\operatorname{Diag}\left(e^{\tau_{0} M_{n}} X_{n l}^{*}\right)^{(t)}$ for $l=1, \ldots, k^{*}$, and $F_{n}^{*}=\left(F_{n 1}^{*}, F_{n 2}^{*}, F_{n 3}^{*}, F_{n 4}^{*}\right)$ with $F_{n 1}^{*}=e^{\tau_{0} M_{n}} X_{n}^{*}, F_{n 2}^{*}=e^{\tau_{0} M_{n}} W_{n} X_{n} \beta_{0}, F_{n 3}^{*}=l_{n}$, and $F_{n 4}^{*}=\operatorname{vec}_{\mathrm{D}}\left(\mathbb{W}_{n}\right)$. Denote $V_{n}^{*}=n \mathrm{E}\left[g_{n}^{*}\left(\gamma_{0}\right) g_{n}^{*^{\prime}}\left(\gamma_{0}\right)\right]$. Then $\hat{\gamma}_{n}^{*}=\min _{\gamma} g_{n}^{*^{\prime}}(\gamma) V_{n}^{*-1} g_{n}^{*}(\gamma)$ is the best GMME within the class of GMMEs with linear and quadratic moments, and $\hat{\gamma}_{n}^{*}$ has the asymptotic distribution that

$$
\sqrt{n}\left(\hat{\gamma}_{n}^{*}-\gamma_{0}\right) \stackrel{d}{\rightarrow} N\left(0, \lim _{n \rightarrow \infty} \Lambda_{n}^{*-1}\right)
$$

\footnotetext{
${ }^{16}$ This pursuit is motivated by that in Liu et al. (2010).

${ }^{17}$ If $M_{n}$ is row-normalized and $X_{n}$ contains an intercept term, $e^{\tau_{0} M_{n}} l_{n}=\sum_{j=0}^{\infty} \frac{1}{j !} \tau_{0}^{j} M_{n}^{j} l_{n}=e^{\tau_{0}} l_{n}$. In this case, $c\left(\tau_{0}\right)=e^{\tau_{0}}$. Otherwise $e^{\tau_{0} M_{n}} X_{n}$ generally does not contain an intercept term.
} 
where $\Lambda_{n}^{*}=G_{n}^{*^{\prime}} V_{n}^{*-1} G_{n}^{*}$ with $G_{n}^{*}=\mathrm{E} \frac{\partial g_{n}^{*}\left(\gamma_{0}\right)}{\partial \gamma^{\prime}}$.

The detailed proof of this proposition is in Appendix C. From the proof, $\Lambda_{n}^{*}$ has the following expression

$$
\Lambda_{n}^{*}=\frac{1}{n}\left(\begin{array}{ccc}
\operatorname{tr}\left(P_{\alpha n}^{* s} \mathbb{W}_{n}\right)+\sigma_{0}^{-2}\left(e^{\tau_{0} M_{n}} W_{n} X_{n} \beta_{0}\right)^{\prime} F_{\alpha n}^{*} & * & * \\
\operatorname{tr}\left(P_{\tau n}^{* s} \mathbb{W}_{n}\right) & \operatorname{tr}\left(P_{\tau n}^{* s} M_{n}\right) & * \\
-\sigma_{0}^{-2}\left(e^{\tau_{0} M_{n}} X_{n}\right)^{\prime} F_{\alpha n}^{*} & 0 & \sigma_{0}^{-2}\left(e^{\tau_{0} M_{n}} X_{n}\right)^{\prime} F_{\beta n}^{*}
\end{array}\right)
$$

where $P_{\alpha n}^{*}=P_{n 1}^{*}-\frac{\left(\eta_{4}-3\right)-\eta_{3}^{2}}{\left(\eta_{4}-1\right)-\eta_{3}^{2}} P_{n 2}^{*}-\frac{\sigma_{0}^{-1} \eta_{3}}{\left(\eta_{4}-1\right)-\eta_{3}^{2}} P_{n 3}^{*}, P_{\tau n}^{*}=M_{n}, P_{\beta n l}^{*}=P_{n, l+4}^{*}$ for $l=1, \ldots, k^{*}, F_{\alpha n}^{*}=$ $\frac{\eta_{4}-1}{\left(\eta_{4}-1\right)-\eta_{3}^{2}} F_{n 2}^{*}-\frac{\eta_{3}^{2}}{\left(\eta_{4}-1\right)-\eta_{3}^{2}} F_{n 3}^{*}\left(\frac{1}{n} l_{n}^{\prime} e^{\tau_{0} M_{n}} W_{n} X_{n} \beta_{0}\right)-\frac{2 \sigma_{0} \eta_{3}}{\left(\eta_{4}-1\right)-\eta_{3}^{2}} F_{n 4}^{*}$,

$$
F_{\beta n}^{*}=\frac{\eta_{4}-1}{\left(\eta_{4}-1\right)-\eta_{3}^{2}} F_{n 1}^{*}-\frac{\eta_{3}^{2}}{\left(\eta_{4}-1\right)-\eta_{3}^{2}} F_{n 3}^{*}\left(\frac{1}{n} l_{n}^{\prime} e^{\tau_{0} M_{n}} X_{n}^{*}\right)
$$

if $e^{\tau_{0} M_{n}} X_{n}$ does not contain an intercept term; otherwise

$$
F_{\beta n}^{*}=\frac{\eta_{4}-1}{\left(\eta_{4}-1\right)-\eta_{3}^{2}} F_{n 1}^{*}\left(I_{k^{*}}, 0_{k^{*} \times 1}\right)+\frac{\eta_{4}-1}{\left(\eta_{4}-1\right)-\eta_{3}^{2}} c\left(\tau_{0}\right) F_{n 3}^{*} e_{k k}^{\prime}-\frac{\eta_{3}^{2}}{\left(\eta_{4}-1\right)-\eta_{3}^{2}} F_{n 3}^{*}\left(\frac{1}{n} l_{n}^{\prime} e^{\tau_{0} M_{n}} X_{n}\right),
$$

where $e_{k j}$ is the $j$ th unit vector in $R^{k}$. From the proof, the best moments in Proposition 8 are equivalent to the use of the following moments

$$
\frac{1}{n}\left(P_{\alpha n}^{*} \epsilon_{n}(\gamma), P_{\tau n}^{*} \epsilon_{n}(\gamma), P_{\beta n 1}^{*} \epsilon_{n}(\gamma) \ldots, P_{\beta n k^{*}}^{*} \epsilon_{n}(\gamma), F_{\alpha n}^{*}, F_{\beta n}^{*}\right)^{\prime} \epsilon_{n}(\gamma)
$$

The above vector relates the moments to the skewness and kurtosis.

In the case of normal disturbances, as $\eta_{3}=\eta_{4}-3=0$, the best moments can be simplified and are equivalent to

$$
\frac{1}{n}\left(\mathbb{W}_{n} \epsilon_{n}(\gamma), M_{n} \epsilon_{n}(\gamma), P_{\beta n 1}^{*} \epsilon_{n}(\gamma) \ldots, P_{\beta n k^{*}}^{*} \epsilon_{n}(\gamma), e^{\tau_{0} M_{n}} W_{n} X_{n} \beta_{0}, e^{\tau_{0} M_{n}} X_{n}\right)^{\prime} \epsilon_{n}(\gamma)
$$

Furthermore, the moments $\left(P_{\beta n 1}^{*} \epsilon_{n}(\gamma) \ldots, P_{\beta n k^{*}}^{*} \epsilon_{n}(\gamma)\right)^{\prime} \epsilon_{n}(\gamma)$ can be shown to be redundant given

$$
g_{n}^{\#}(\gamma)=\frac{1}{n}\left(\mathbb{W}_{n} \epsilon_{n}(\gamma), M_{n} \epsilon_{n}(\gamma), e^{\tau_{0} M_{n}} W_{n} X_{n} \beta_{0}, e^{\tau_{0} M_{n}} X_{n}\right)^{\prime} \epsilon_{n}(\gamma)
$$

by an argument similar to the proof of Proposition 8. This result can also be shown by using the generalized Cauchy-Schwarz inequality, as in subsequent section. 
The $G_{n}$ in Proposition 7 can be written as

$$
G_{n}=\frac{1}{n \sigma_{0}^{2}}\left(\begin{array}{cc}
\frac{\sqrt{2} \sigma_{0}^{2}}{2} \omega_{n} & 0 \\
0 & \sigma_{0} F_{n}
\end{array}\right)^{\prime} G_{1 n}
$$

where

$$
\begin{aligned}
G_{1 n} & =\left(\begin{array}{ccc}
\frac{\sqrt{2} \sigma_{0}^{2}}{2} \operatorname{vec}\left(\mathbb{W}_{n}^{s}\right) & \frac{\sqrt{2} \sigma_{0}^{2}}{2} \operatorname{vec}\left(M_{n}^{s}\right) & 0 \\
\sigma_{0} \mathbb{W}_{n} e^{\tau_{0} M_{n}} X_{n} \beta_{0} & 0 & -\sigma_{0} e^{\tau_{0} M_{n}} X_{n}
\end{array}\right) \\
& =\left(\begin{array}{cccc}
\frac{\sqrt{2} \sigma_{0}^{2}}{2} \operatorname{vec}\left(\mathbb{W}_{n}^{s}\right) & \frac{\sqrt{2} \sigma_{0}^{2}}{2} \operatorname{vec}\left(M_{n}^{s}\right) & 0 & 0 \\
0 & 0 & \sigma_{0} \mathbb{W}_{n} e^{\tau_{0} M_{n}} X_{n} \beta_{0} & \sigma_{0} e^{\tau_{0} M_{n}} X_{n}
\end{array}\right)\left(\begin{array}{ccc}
1 & 0 & 0 \\
0 & 1 & 0 \\
1 & 0 & 0 \\
0 & 0 & -I_{k}
\end{array}\right) .
\end{aligned}
$$

When $\epsilon_{n i}$ 's are normal, $\mu_{3}=\mu_{4}-3 \sigma_{0}^{4}=0$. Furthermore, even under non-normal disturbances, if $P_{n 1}, \ldots, P_{n, k_{p}}$ are chosen to have zero diagonal, then $\omega_{n d}=0$. For those cases, $V_{n}$ in Proposition 7 reduces to

$$
V_{n}=\frac{1}{n}\left(\begin{array}{cc}
\frac{\sigma_{0}^{4}}{2} \omega_{n}^{\prime} \omega_{n} & 0 \\
0 & \sigma_{0}^{2} F_{n}^{\prime} F_{n}
\end{array}\right)=\frac{1}{n}\left(\begin{array}{cc}
\frac{\sqrt{2} \sigma_{0}^{2}}{2} \omega_{n} & 0 \\
0 & \sigma_{0} F_{n}
\end{array}\right)^{\prime}\left(\begin{array}{cc}
\frac{\sqrt{2} \sigma_{0}^{2}}{2} \omega_{n} & 0 \\
0 & \sigma_{0} F_{n}
\end{array}\right) .
$$

Thus for those cases, $G_{n}^{\prime} V_{n}^{-1} G_{n} \leq \Lambda_{n}$ by the generalized Cauchy-Schwarz inequality, where $\Lambda_{n}=\frac{1}{n \sigma_{0}^{4}} G_{1 n}^{\prime} G_{1 n}$. As $\mathbb{W}_{n}$ and $M_{n}$ both have zero traces, when the moment vector is $g_{n}^{\#}(\gamma)$ in (31), $G_{n}^{\prime} V_{n}^{-1} G_{n}=\Lambda_{n}$. Thus the best moment vector is $g_{n}^{\#}(\gamma)$ in (31) when $\epsilon_{n i}$ 's are normal. When $\tau_{0}=0$ or $W_{n}$ and $M_{n}$ can commute, $\mathbb{W}_{n}=W_{n}$ has a zero diagonal; and the best moment vector, with the restriction that $P_{n i}$ 's have zero diagonals, is ${ }^{18}$

$$
g_{n, d}^{\#}(\gamma)=\frac{1}{n}\left(W_{n} \epsilon_{n}(\gamma), M_{n} \epsilon_{n}(\gamma), e^{\tau_{0} M_{n}} W_{n} X_{n} \beta_{0}, e^{\tau_{0} M_{n}} X_{n}\right)^{\prime} \epsilon_{n}(\gamma)
$$

By comparing (32) and (19), the asymptotic VC matrix $\Lambda_{n}^{-1}$ for the best GMME in the case of normal disturbances is the same as that for the MLE of $\gamma$. It is of interest to note that, for the case with non-normal disturbances, when $\tau_{0}=0$, or $W_{n}$ and $M_{n}$ can commute, the QMLE of $\gamma$ happens to be asymptotically efficient within the class of GMMEs with linear and quadratic moments where the quadratic matrices $P_{n i}$ 's have zero diagonals.

\footnotetext{
${ }^{18}$ When $\tau_{0}=0$ or $W_{n}=M_{n}$, the moment $\frac{1}{n} \epsilon_{n}^{\prime}(\gamma) M_{n} \epsilon_{n}(\gamma)$ should be deleted.
} 
Corollary 1. Suppose that Assumptions 1, 2, 5 and 12-15 hold.

(i) When the disturbances $\epsilon_{n i}$ 's are normal, for the class of GMMEs with linear and quadratic moments where the quadratic matrices $P_{n i}$ 's have zero traces, the best GMME is the optimal GMME with the moment vector $g_{n}^{\#}(\gamma)$ in $(31)$;

(ii) When $\tau_{0}=0$, or $W_{n}$ and $M_{n}$ can commute, for the class of GMMEs with linear and quadratic moments where the quadratic matrices $P_{n i}$ 's have zero diagonals, the best GMME is the optimal GMME with the moment vector $g_{n, d}^{\#}(\gamma)$ in (33).

The best moments in the case of normal disturbances are of interest to be compared with those for the SARAR model. For the latter model, the best instruments are $R_{n}\left[X_{n}, W_{n} S_{n}^{-1} X_{n} \beta_{0}\right]$ and the matrices for the best quadratic moments are $R_{n} W_{n} S_{n}^{-1} R_{n}^{-1}-I_{n} \operatorname{tr}\left(W_{n} S_{n}^{-1}\right) / n$ and $M_{n} R_{n}^{-1}-I_{n} \operatorname{tr}\left(M_{n} R_{n}^{-1}\right) / n$, where $R_{n}=I_{n}-\rho_{0} M_{n}$ and $S_{n}=I_{n}-\lambda_{0} W_{n}$. Thus, in addition to $X_{n}$ and $W_{n} X_{n}$, higher order spatially lagged $X_{n}$, i.e., $W_{n}^{2} X_{n}, W_{n}^{3} X_{n}$, etc., will provide additional information. For the quadratic moments, spatial weights matrices of higher order, namely, $W_{n}^{2}, W_{n}^{3}$, etc., from which the average of their diagonal elements is subtracted from each diagonal element, can be used as additional orthogonal conditions. On the other hand, the best instruments and quadratic moments for the $\operatorname{MESS}(1,1)$ rely simply on spatial weights matrices of the first order, i.e., $W_{n}$ and $M_{n}$. Note also that when there is no MESS process in the disturbances, the moment vector for the best GMME in the case of normal disturbances can be simply taken as $\frac{1}{n}\left[\epsilon_{n}^{\prime}(\gamma) W_{n} \epsilon_{n}(\gamma), \epsilon_{n}^{\prime}(\gamma)\left(W_{n} X_{n}, X_{n}\right)_{I N}\right]^{\prime}$, where $\left(W_{n} X_{n}, X_{n}\right)_{I N}$ denotes the independent columns of $\left(W_{n} X_{n}, X_{n}\right) .{ }^{19}$ Thus it has a simple form which does not involve any unknown parameter. By contrast, the moment vector for the best optimal GMME of the SAR model can be taken as $\frac{1}{n}\left[\epsilon_{n}^{\prime}(\gamma)\left(W_{n} S_{n}^{-1}-\right.\right.$ $\left.\left.I_{n} \operatorname{tr}\left(W_{n} S_{n}^{-1}\right) / n\right) \epsilon_{n}(\gamma), \epsilon_{n}^{\prime}(\gamma)\left(W_{n} S_{n}^{-1} X_{n}, X_{n}\right)_{I N}\right]^{\prime}$, which involves the unknown parameter $\lambda_{0}$ in the matrix inverse $S_{n}^{-1}$.

There exists a link between the MLE (or QMLE) and moment conditions. The first order conditions for the MLE using the function $Q_{n}(\gamma)$ can be written as

$$
\begin{aligned}
& \frac{\partial Q_{n}(\gamma)}{\partial \alpha}=2\left(e^{\tau M_{n}} W_{n} X_{n} \beta\right)^{\prime} \epsilon_{n}(\gamma)+2 \epsilon_{n}^{\prime}(\gamma) e^{\tau M_{n}} W_{n} e^{-\tau M_{n}} \epsilon_{n}(\gamma) \\
& \frac{\partial Q_{n}(\gamma)}{\partial \tau}=2 \epsilon_{n}^{\prime}(\gamma) M_{n} \epsilon_{n}(\gamma) \\
& \frac{\partial Q_{n}(\gamma)}{\partial \beta}=-2\left(e^{\tau M_{n}} X_{n}\right)^{\prime} \epsilon_{n}(\gamma) .
\end{aligned}
$$

\footnotetext{
${ }^{19}$ If $W_{n}$ is row normalized and $X_{n}$ contains an intercept, as $W_{n} l_{n}=l_{n}$, only one of the two intercepts should be included in $\left(W_{n} X_{n}, X_{n}\right)$.
} 
Thus the underlying moments integrated by the MLE are also the linear moments with instruments from $e^{\tau_{0} M_{n}} X_{n}$ and $e^{\tau_{0} M_{n}} W_{n} X_{n}$, and the quadratic moments with the matrices $\mathbb{W}_{n}$ and $M_{n}$. The matrix $e^{\tau_{0} M_{n}}$ in front of $X_{n}$ and $W_{n} X_{n}$ is a transformation for the MESS disturbances. When the likelihood function is correctly specified under the normal disturbances, the combinations of linear and quadratic moments in (34)-(36) are the efficient ones. But they might not be so when the likelihood function is only a quasi one. The optimal GMME employs an optimal weighting matrix when using the moments $g_{n}^{\#}(\gamma)$, but the QMLE might not. Thus a best GMME within the class of linear and quadratic moments can be more efficient asymptotically than the QMLE when the disturbances are non-normal, $\tau_{0} \neq 0$ or $W_{n}$ and $M_{n}$ cannot commute. This can be shown analytically. Let

$$
h_{n}(\gamma)=\frac{2}{n}\left(\left(e^{\tau_{0} M_{n}} W_{n} X_{n} \beta_{0}\right)^{\prime} \epsilon_{n}(\gamma)+\epsilon_{n}^{\prime}(\gamma) W_{n} \epsilon_{n}(\gamma), \epsilon_{n}^{\prime}(\gamma) M_{n} \epsilon_{n}(\gamma),-\epsilon_{n}^{\prime}(\gamma) e^{\tau_{0} M_{n}} X_{n}\right)^{\prime}=A g_{n}^{\#}(\gamma)
$$

where

$$
A=2\left(\begin{array}{cccc}
1 & 0 & 1 & 0 \\
0 & 1 & 0 & 0 \\
0 & 0 & 0 & -I_{k}
\end{array}\right)
$$

The $h_{n}(\gamma)$ and $\frac{1}{n} \frac{\partial Q_{n}(\gamma)}{\partial \gamma}$ have a similar structure: replacing $\tau_{0}$ in the components $e^{\tau_{0} M_{n}} W_{n} X_{n} \beta_{0}$ and $e^{\tau_{0} M_{n}} X_{n}$ in $h_{n}(\gamma)$ by $\tau$ yields $\frac{1}{n} \frac{\partial Q_{n}(\gamma)}{\partial \gamma}$. It is obvious that $\mathrm{E} \frac{\partial h_{n}\left(\gamma_{0}\right)}{\partial \gamma^{\prime}}=\frac{1}{n} \mathrm{E}\left(\frac{\partial^{2} Q_{n}\left(\gamma_{0}\right)}{\partial \gamma \partial \gamma^{\prime}}\right)$ and $\mathrm{E}\left(n h_{n}\left(\gamma_{0}\right) h_{n}^{\prime}\left(\gamma_{0}\right)\right)=$ $\frac{1}{n} \mathrm{E}\left(\frac{\partial Q_{n}\left(\gamma_{0}\right)}{\partial \gamma} \frac{\partial Q_{n}\left(\gamma_{0}\right)}{\partial \gamma^{\prime}}\right)$. Thus, by Proposition 4, the asymptotic VC matrix for the QMLE of $\gamma$ is equal to $\lim _{n \rightarrow \infty}\left(\mathrm{E} \frac{\partial h_{n}\left(\gamma_{0}\right)}{\partial \gamma^{\prime}}\right)^{-1} \mathrm{E}\left(n h_{n}\left(\gamma_{0}\right) h_{n}^{\prime}\left(\gamma_{0}\right)\right)\left(\mathrm{E} \frac{\partial h_{n}\left(\gamma_{0}\right)}{\partial \gamma}\right)^{-1}$. Therefore,

$$
\begin{aligned}
& \left(\mathrm{E} \frac{\partial h_{n}\left(\gamma_{0}\right)}{\partial \gamma^{\prime}}\right)^{-1} \mathrm{E}\left(h_{n}\left(\gamma_{0}\right) h_{n}^{\prime}\left(\gamma_{0}\right)\right)\left(\mathrm{E} \frac{\partial h_{n}^{\prime}\left(\gamma_{0}\right)}{\partial \gamma}\right)^{-1} \\
& =\left[\left(\mathrm{E} \frac{\partial h_{n}^{\prime}\left(\gamma_{0}\right)}{\partial \gamma}\right)\left[\mathrm{E}\left(h_{n}\left(\gamma_{0}\right) h_{n}^{\prime}\left(\gamma_{0}\right)\right)\right]^{-1}\left(\mathrm{E} \frac{\partial h_{n}\left(\gamma_{0}\right)}{\partial \gamma^{\prime}}\right)\right]^{-1} \\
& =\left[G_{n}^{\#^{\prime}}\left(\gamma_{0}\right) A^{\prime}\left[A \mathrm{E}\left(g_{n}^{\#}\left(\gamma_{0}\right) g_{n}^{\#^{\prime}}\left(\gamma_{0}\right)\right) A^{\prime}\right]^{-1} A G_{n}^{\#}\left(\gamma_{0}\right)\right]^{-1} \\
& \geq\left[G_{n}^{\#^{\prime}}\left(\gamma_{0}\right)\left[\mathrm{E}\left(g_{n}^{\#}\left(\gamma_{0}\right) g_{n}^{\#^{\prime}}\left(\gamma_{0}\right)\right)\right]^{-1} G_{n}^{\#}\left(\gamma_{0}\right)\right]^{-1},
\end{aligned}
$$

by the generalized Cauchy-Schwarz inequality, where $G_{n}^{\#}\left(\gamma_{0}\right)=\mathrm{E} \frac{\partial g_{n}^{\#}\left(\gamma_{0}\right)}{\partial \gamma^{\prime}}$. The last term above is the asymptotic VC matrix of the feasible optimal GMME with the moment vector $g_{n}^{\#}(\gamma)$. The inequality in (38) becomes an equality if there is a matrix $\Delta_{g h}$ such that $G_{n}^{\#}\left(\gamma_{0}\right)=\mathrm{E}\left(g_{n}^{\#}\left(\gamma_{0}\right) g_{n}^{\#^{\prime}}\left(\gamma_{0}\right)\right) A^{\prime} \Delta_{g h}$. From 
Proposition 6, we have

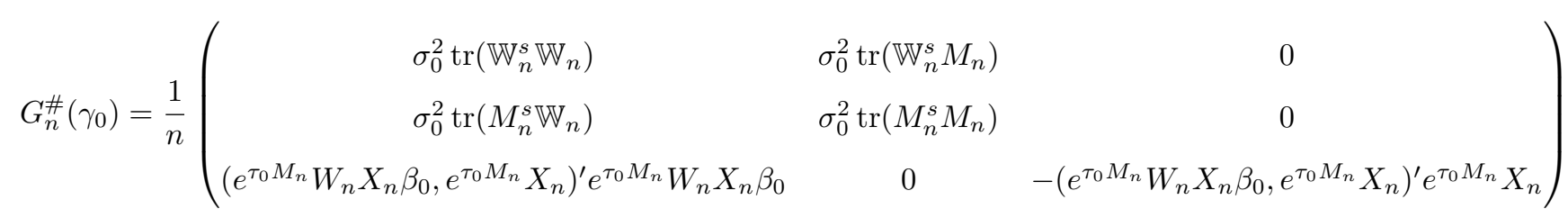

and

$$
\begin{aligned}
& \mathrm{E}\left(g_{n}^{\#}\left(\gamma_{0}\right) g_{n}^{\#^{\prime}}\left(\gamma_{0}\right)\right) A^{\prime}=\frac{2 \sigma_{0}^{2}}{n} G_{n}^{\#}+ \\
& \quad \frac{2}{n^{2}}\left(\begin{array}{ccc}
\left(\mu_{4}-3 \sigma_{0}^{4}\right) \operatorname{vec}_{\mathrm{D}}^{\prime}\left(\mathbb{W}_{n}\right) \operatorname{vec}_{\mathrm{D}}\left(\mathbb{W}_{n}\right)+\mu_{3} \operatorname{vec}_{\mathrm{D}}{ }^{\prime}\left(\mathbb{W}_{n}\right) e^{\tau_{0} M_{n}} W_{n} X_{n} \beta_{0} & 0 & -\mu_{3} \operatorname{vec}_{\mathrm{D}}{ }^{\prime}\left(\mathbb{W}_{n}\right) e^{\tau_{0} M_{n}} X_{n} \\
0 & 0 & 0 \\
\mu_{3}\left(e^{\tau_{0} M_{n}} W_{n} X_{n} \beta_{0}, e^{\tau_{0} M_{n}} X_{n}\right)^{\prime} \operatorname{vec}_{\mathrm{D}}\left(\mathbb{W}_{n}\right) & 0 & 0
\end{array}\right) .
\end{aligned}
$$

When $\tau_{0}=0 ; W_{n}$ and $M_{n}$ can commute; or $\mu_{3}=\mu_{4}-3 \sigma_{0}^{4}=0$, we have $\Delta_{g h}=\frac{n}{2 \sigma_{0}^{2}} I_{k+2}$. Except for those cases, $\Delta_{g h}$ may not exist. As $g_{n}^{\#}(\gamma)$ in $(31)$ is only a special case of linear and quadratic moments, the best GMME in Proposition 8 can be more efficient asymptotically than the QMLE.

The best moment vector $g_{n}^{*}(\gamma)$ and the optimal weighting matrix $V_{n}^{*-1}$ involve unknown parameters. In practice, $g_{n}^{*}(\gamma)$ and $V_{n}^{*-1}$ can be estimated using initial consistent estimates and a feasible best GMME can be derived. The following proposition shows that such a feasible best GMME has the same asymptotic distribution as the best GMME in Proposition 8.

Proposition 9. Suppose that Assumptions 1, 2, 5 and 12-15 hold. Let $\hat{\gamma}_{n}, \hat{\sigma}_{n}^{2}, \hat{\mu}_{3 n}$ and $\hat{\mu}_{4 n}$ be, respectively, $\sqrt{n}$-consistent estimators of $\gamma_{0}, \sigma_{0}^{2}, \mu_{3}$ and $\mu_{4}$. The $\hat{P}_{n 1}^{*}, \ldots, \hat{P}_{n, k^{*}+4}^{*}, \hat{F}_{n}^{*}, \hat{F}_{1 n}^{*}, \ldots, \hat{F}_{n 4}^{*}$ and $\hat{V}_{n}^{*}$ denote the matrices derived when the unknown parameters in $P_{n 1}^{*}, \ldots, P_{n, k^{*}+4}^{*}, F_{n}^{*}, F_{1 n}^{*}, \ldots, F_{n 4}^{*}$ and $V_{n}^{*}$ are replaced by the corresponding consistent estimators. Then the feasible best GMME $\hat{\gamma}_{n, f}^{*}=$ $\min _{\gamma} \hat{g}_{n}^{*^{\prime}}(\gamma) \hat{V}_{n}^{*-1} \hat{g}_{n}^{*}(\gamma)$, where $\hat{g}_{n}^{*}(\gamma)=\frac{1}{n}\left(\hat{P}_{n 1}^{*} \epsilon(\gamma), \ldots, \hat{P}_{n, k^{*}+4}^{*} \epsilon(\gamma), \hat{F}_{n}^{*}\right)^{\prime} \epsilon(\gamma)$, has the same asymptotic distribution as $\hat{\gamma}_{n}^{*}=\min _{\gamma} g_{n}^{*^{\prime}}(\gamma) V_{n}^{*-1} g_{n}^{*}(\gamma)$.

\subsubsection{GMME: Heteroskedastic Case}

When the disturbances are independent but may have different variances, the GMME can be consistent when the matrices $P_{n i}$ 's have zero diagonals.

Assumption 16. The $n$-dimensional square matrices $P_{n 1}, \ldots, P_{n, k_{p}}$ have zero diagonals and are bounded in both row and column sum norms. Elements of $F_{n}$ are uniformly bounded constants. 
By taking into account variances of disturbances, the identification condition is similarly derived as that in the homoskedastic case.

Assumption 17. $\lim _{n \rightarrow \infty} \frac{1}{n}\left[\operatorname{tr}\left(e^{\left(\tau-\tau_{0}\right) M_{n}^{\prime}} P_{n 1} e^{\left.\left(\tau-\tau_{0}\right) M_{n} \Sigma_{n}\right)}, \ldots, \operatorname{tr}\left(e^{\left(\tau-\tau_{0}\right) M_{n}^{\prime}} P_{n, k_{p}} e^{\left.\left.\left(\tau-\tau_{0}\right) M_{n} \Sigma_{n}\right)\right]} \neq 0\right.\right.\right.$ for any $\tau \neq \tau_{0}$, and $F_{n}$ may be written as $F_{n}=\left(F_{1 n}, F_{2 n}\right)$ such that $\lim _{n \rightarrow \infty} \frac{1}{n} F_{2 n}^{\prime} e^{\tau M_{n}} X_{n}$ is nonsingular for any $\tau \in[-\delta, \delta]$, and $\lim _{n \rightarrow \infty} \frac{1}{n} F_{1 n}^{\prime} H_{1 n}(\tau) e^{\tau M_{n}} e^{\eta W_{n}} X_{n} \beta_{0} \neq 0$ for any $\eta \neq 0$ and $\tau \in[-\delta, \delta]$.

Proposition 10. Under Assumptions 1, 2, 8, 14, 16 and 17, the GMM estimator $\hat{\gamma}_{n}$ from the minimization of $g_{n}^{\prime}(\gamma) a_{n} a_{n}^{\prime} g_{n}(\gamma)$ is a consistent estimator of $\gamma_{0}$, and

$$
\sqrt{n}\left(\hat{\gamma}_{n}-\gamma_{0}\right) \stackrel{d}{\rightarrow} N\left(0, \lim _{n \rightarrow \infty}\left(G_{n}^{\prime} a_{n} a_{n}^{\prime} G_{n}\right)^{-1} G_{n}^{\prime} a_{n} a_{n}^{\prime} V_{n} a_{n} a_{n}^{\prime} G_{n}\left(G_{n}^{\prime} a_{n} a_{n}^{\prime} G_{n}\right)^{-1}\right)
$$

where

$$
V_{n}=n \mathrm{E}\left[g_{n}\left(\gamma_{0}\right) g_{n}^{\prime}\left(\gamma_{0}\right)\right]=\frac{1}{n}\left(\begin{array}{cc}
\frac{1}{2} \omega_{n}^{\prime} \omega_{n} & 0 \\
0 & F_{n}^{\prime} \Sigma_{n} F_{n}
\end{array}\right)
$$

and

$$
G_{n}=\mathrm{E} \frac{\partial g_{n}\left(\gamma_{0}\right)}{\partial \gamma^{\prime}}=\frac{1}{n}\left(\begin{array}{ccc}
\frac{1}{2} \omega_{n}^{\prime} \operatorname{vec}\left(\Sigma_{n}^{1 / 2}\left(\Sigma_{n}^{-1} \mathbb{W}_{n}\right)^{s} \Sigma_{n}^{1 / 2}\right) & \frac{1}{2} \omega_{n}^{\prime} \operatorname{vec}\left(\Sigma_{n}^{1 / 2}\left(\Sigma_{n}^{-1} M_{n}\right)^{s} \Sigma_{n}^{1 / 2}\right) & 0 \\
F_{n}^{\prime} \mathbb{W}_{n} e^{\tau_{0} M_{n}} X_{n} \beta_{0} & 0 & -F_{n}^{\prime} e^{\tau_{0} M_{n}} X_{n}
\end{array}\right)
$$

with $\omega_{n}=\left(\operatorname{vec}\left(\Sigma_{n}^{1 / 2} P_{n 1}^{s} \Sigma_{n}^{1 / 2}\right), \ldots, \operatorname{vec}\left(\Sigma_{n}^{1 / 2} P_{n, k_{p}}^{s} \Sigma_{n}^{1 / 2}\right)\right)$, under the condition that $\lim _{n \rightarrow \infty} a_{n}^{\prime} G_{n}$ exists and has the full rank $k+2$.

The $V_{n}$ does not involve the third and fourth moments of the disturbances, as the matrices in the quadratic forms of disturbances in $g_{n}\left(\gamma_{0}\right)$ have zero diagonals. An optimal GMME can also be formulated.

Assumption 18. The limits of $\frac{1}{n} \omega_{n}^{\prime} \omega_{n}$ and $\frac{1}{n} F_{n}^{\prime} \Sigma_{n} F_{n}$ exist and are nonsingular.

A consistent estimator for $V_{n}$ is the matrix $\hat{V}_{n}$ derived by replacing the $\Sigma_{n}$ in $V_{n}$ by $\hat{\Sigma}_{n}=\operatorname{Diag}\left(\hat{\epsilon}_{n 1}^{2}, \ldots, \hat{\epsilon}_{n n}^{2}\right)$, where $\hat{\epsilon}_{n i}$ 's are the residuals from an initial GMM estimation. Under Assumption 18, the limiting inverse of $V_{n}$ exists. Then the objective function for the feasible optimal GMME is $g_{n}^{\prime}(\gamma) \hat{V}_{n}^{-1} g_{n}(\gamma)$.

Proposition 11. Under Assumptions 1, 2, 8, 14 and 16-18, the feasible optimal GMME $\hat{\gamma}_{n, o}$ from the minimization of $g_{n}^{\prime}(\gamma) \hat{V}_{n}^{-1} g_{n}(\gamma)$ is a consistent estimator of $\gamma_{0}$, and

$$
\sqrt{n}\left(\hat{\gamma}_{n, o}-\gamma_{0}\right) \stackrel{d}{\rightarrow} N\left(0, \lim _{n \rightarrow \infty}\left(G_{n}^{\prime} V_{n}^{-1} G_{n}\right)^{-1}\right) .
$$


Note that $\operatorname{tr}\left(\Sigma_{n} P_{n i}^{s} \Sigma_{n}\left(\Sigma_{n}^{-1} \mathbb{W}_{n}\right)^{s}\right)=\operatorname{tr}\left(\Sigma_{n} P_{n i}^{s} \Sigma_{n}\left(\Sigma_{n}^{-1}\left(\mathbb{W}_{n}-\operatorname{Diag}\left(\mathbb{W}_{n}\right)\right)\right)^{s}\right)$ as $P_{n i}^{s}$ has a zero diagonal and $\Sigma_{n}$ is a diagonal matrix, then $G_{n}$ may be written as

$$
G_{n}=\frac{1}{n}\left(\begin{array}{cc}
\frac{\sqrt{2}}{2} \omega_{n} & 0 \\
0 & \Sigma_{n}^{1 / 2} F_{n}
\end{array}\right)^{\prime} G_{1 n}
$$

where

$$
G_{1 n}=\left(\begin{array}{ccc}
\frac{\sqrt{2}}{2} \operatorname{vec}\left(\Sigma_{n}^{1 / 2}\left(\Sigma_{n}^{-1}\left(\mathbb{W}_{n}-\operatorname{Diag}\left(\mathbb{W}_{n}\right)\right)\right)^{s} \Sigma_{n}^{1 / 2}\right) & \frac{\sqrt{2}}{2} \operatorname{vec}\left(\Sigma_{n}^{1 / 2}\left(\Sigma_{n}^{-1} M_{n}\right)^{s} \Sigma_{n}^{1 / 2}\right) & 0 \\
\Sigma_{n}^{-1 / 2} \mathbb{W}_{n} e^{\tau_{0} M_{n}} X_{n} \beta_{0} & 0 & -\Sigma_{n}^{-1 / 2} e^{\tau_{0} M_{n}} X_{n}
\end{array}\right)
$$

Thus $G_{n}^{\prime} V_{n}^{-1} G_{n} \leq \Lambda_{n}$ by the generalized Cauchy-Schwarz inequality, where $\Lambda_{n}=\frac{1}{n} G_{1 n}^{\prime} G_{1 n}$. When the moment vector $g_{n}(\gamma)$ is equal to $g_{n}^{*}(\gamma)=\frac{1}{n}\left[\epsilon_{n}^{\prime}(\gamma) \Sigma_{n}^{-1}\left(\mathbb{W}_{n}-\operatorname{Diag}\left(\mathbb{W}_{n}\right)\right) \epsilon_{n}(\gamma), \epsilon_{n}^{\prime}(\gamma) \Sigma_{n}^{-1} M_{n} \epsilon_{n}(\gamma), \epsilon_{n}^{\prime}(\gamma) F_{n}^{*}\right]^{\prime}$ with $F_{n}^{*}=\Sigma_{n}^{-1}\left[\mathbb{W}_{n} e^{\tau_{0} M_{n}} X_{n} \beta_{0}, e^{\tau_{0} M_{n}} X_{n}\right], G_{n}^{\prime} V_{n}^{-1} G_{n}=\Lambda_{n}$. Therefore, if the variances $\sigma_{n i}^{2}$ 's can be consistently estimated, e.g., when we have a parametric model for the variances, then we may have a feasible best optimal GMME. ${ }^{20}$ Let $\hat{\gamma}_{n}$ be an initial consistent estimator of $\gamma_{0}$, $\hat{\mathbb{W}}_{n}=e^{\hat{\tau}_{n} M_{n}} W_{n} e^{-\hat{\tau}_{n} M_{n}}$ and $\hat{F}_{n}^{*}=\hat{\Sigma}_{n}^{-1}\left[\hat{\mathbb{W}}_{n} e^{\hat{\tau}_{n} M_{n}} X_{n} \hat{\beta}_{n}, e^{\hat{\tau}_{n} M_{n}} X_{n}\right]$ with $\hat{\Sigma}_{n}$ being a diagonal matrix containing consistent estimators for the variances. Then the objective function for the feasible best optimal GMME is $\hat{g}_{n}^{*^{\prime}}(\gamma) \hat{V}_{n}^{*-1} \hat{g}_{n}^{*}(\gamma)$, where $\hat{g}_{n}^{*}(\gamma)=\frac{1}{n}\left(\epsilon_{n}^{\prime}(\gamma) \hat{\Sigma}_{n}^{-1}\left(\hat{\mathbb{W}}_{n}-\operatorname{Diag}\left(\hat{\mathbb{W}}_{n}\right)\right) \epsilon_{n}(\gamma), \epsilon_{n}^{\prime}(\gamma) \hat{\Sigma}_{n}^{-1} M_{n} \epsilon_{n}(\gamma), \epsilon_{n}^{\prime}(\gamma) \hat{F}_{n}^{*}\right)^{\prime}$ and

$$
\hat{V}_{n}^{*}=\frac{1}{n}\left(\begin{array}{ccc}
\frac{1}{2} \operatorname{tr}\left[\hat{\Sigma}_{n}\left(\hat{\Sigma}_{n}^{-1}\left(\hat{\mathbb{W}}_{n}-\operatorname{Diag}\left(\hat{\mathbb{W}}_{n}\right)\right)\right)^{s} \hat{\Sigma}_{n}\left(\hat{\Sigma}_{n}^{-1} \hat{\mathbb{W}}_{n}\right)^{s}\right] & \frac{1}{2} \operatorname{tr}\left[\hat{\Sigma}_{n}\left(\hat{\Sigma}_{n}^{-1} \hat{\mathbb{W}}_{n}\right)^{s} \hat{\Sigma}_{n}\left(\hat{\Sigma}_{n}^{-1} M_{n}\right)^{s}\right] & 0 \\
\frac{1}{2} \operatorname{tr}\left[\hat{\Sigma}_{n}\left(\hat{\Sigma}_{n}^{-1} \hat{\mathbb{W}}_{n}\right)^{s} \hat{\Sigma}_{n}\left(\hat{\Sigma}_{n}^{-1} M_{n}\right)^{s}\right] & \frac{1}{2} \operatorname{tr}\left[\hat{\Sigma}_{n}\left(\hat{\Sigma}_{n}^{-1} M_{n}\right)^{s} \hat{\Sigma}_{n}\left(\hat{\Sigma}_{n}^{-1} M_{n}\right)^{s}\right] & 0 \\
0 & 0 & \hat{F}_{n}^{*^{\prime}} \hat{\Sigma}_{n} \hat{F}_{n}^{*}
\end{array}\right)
$$

If the elements of $\Sigma_{n}$ cannot be consistently estimated, we do not have a feasible best GMME, e.g., for the unknown heteroskedastic case, $\Sigma_{n}$ with $n$ parameters cannot be consistently estimated. However, we may use the moment vector

$$
\hat{g}_{n, d}^{\#}(\gamma)=\frac{1}{n}\left[\epsilon_{n}^{\prime}(\gamma)\left(\hat{\mathbb{W}}_{n}-\operatorname{Diag}\left(\hat{\mathbb{W}}_{n}\right)\right) \epsilon_{n}(\gamma), \epsilon_{n}^{\prime}(\gamma) M_{n} \epsilon_{n}(\gamma), \epsilon_{n}^{\prime}(\gamma)\left[\hat{\mathbb{W}}_{n} e^{\hat{\tau}_{n} M_{n}} X_{n} \hat{\beta}, e^{\hat{\tau}_{n} M_{n}} X_{n}\right]\right]^{\prime}
$$

\footnotetext{
${ }^{20}$ For the SARAR model with heteroskedasticity, we have also found that the best instruments are $\Sigma_{n}^{-1} R_{n}\left[X_{n}, W_{n} S_{n}^{-1} X_{n} \beta_{0}\right]$ and the matrices for the quadratic moments are $\Sigma_{n}^{-1}\left[R_{n} W_{n} S_{n}^{-1} R_{n}^{-1}-\operatorname{Diag}\left(R_{n} W_{n} S_{n}^{-1} R_{n}^{-1}\right)\right]$ and $\Sigma_{n}^{-1}\left[M_{n} R_{n}^{-1}-\right.$ $\left.\operatorname{Diag}\left(M_{n} R_{n}^{-1}\right)\right]$. For the SAR model with heteroskedasticity, Lin and Lee (2010) have not discussed the possible best GMME by the generalized Cauchy-Schwarz inequality as above.
} 
and implement a feasible optimal GMM estimation. A special case of interest is when $\tau_{0}=0$ or $W_{n}$ and $M_{n}$ can commute, and the QMLE can be consistent. In that case, $\hat{g}_{n, d}^{\#}(\gamma)$ reduces to

$$
\hat{g}_{n, d}^{\#}(\gamma)=\frac{1}{n}\left[\epsilon_{n}^{\prime}(\gamma) W_{n} \epsilon_{n}(\gamma), \epsilon_{n}^{\prime}(\gamma) M_{n} \epsilon_{n}(\gamma), \epsilon_{n}^{\prime}(\gamma)\left[W_{n} e^{\hat{\tau}_{n} M_{n}} X_{n} \hat{\beta}_{n}, e^{\hat{\tau}_{n} M_{n}} X_{n}\right]\right]^{\prime}
$$

It can be shown, as for the proof of Proposition 9, that the optimal GMME using the moment vector $\hat{g}_{n, d}^{\#}(\gamma)$ has the same asymptotic distribution as that using the moment vector $g_{n, d}^{\#}(\gamma)$ in (33). As shown in (34)-(36), the QMLE also integrates those moments in $g_{n, d}^{\#}(\gamma)$. But the optimal GMME using the moment vector $g_{n, d}^{\#}(\gamma)$ is at least as efficient as the QMLE and can be more efficient than the QMLE asymptotically, according to (38). For the QMLE to be as efficient as the optimal GMME, there must exist a matrix $\Delta_{g h}$ such that $G_{n, d}^{\#}\left(\gamma_{0}\right)=\mathrm{E}\left(g_{n, d}^{\#}\left(\gamma_{0}\right) g_{n, d}^{\#^{\prime}}\left(\gamma_{0}\right)\right) A^{\prime} \Delta_{g h}$, where $G_{n, d}^{\#}\left(\gamma_{0}\right)=\mathrm{E} \frac{\partial g_{n, d}^{\#}\left(\gamma_{0}\right)}{\partial \gamma^{\prime}}$ and the matrix $A$ is given in (37). From Proposition 11, we have

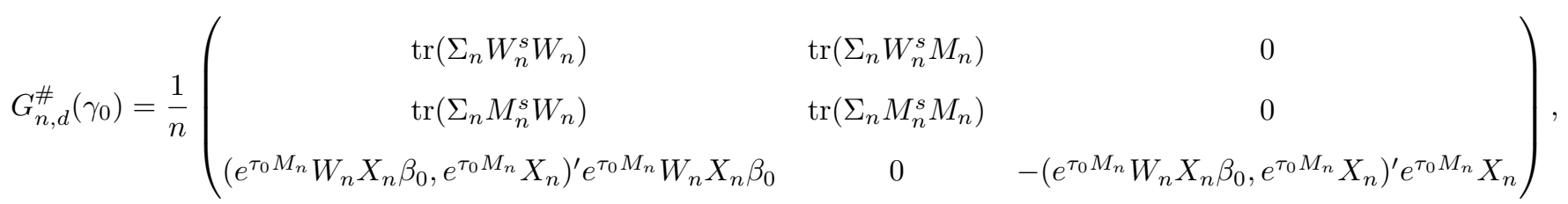

and

$$
\begin{aligned}
& \mathrm{E}\left(g_{n, d}^{\#}\left(\gamma_{0}\right) g_{n, d}^{\# \prime}\left(\gamma_{0}\right)\right) A^{\prime}
\end{aligned}
$$

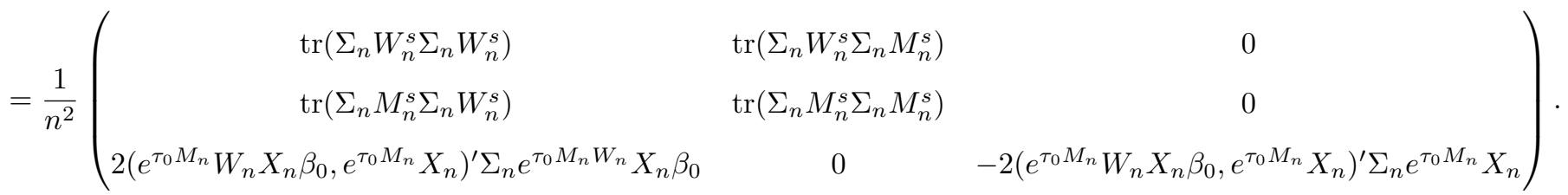

Thus, under unknown heteroskedasticity, generally there does not exist such a $\Delta_{g h}$. Then the optimal GMME using the moment vector $\hat{g}_{n, d}^{\#}(\gamma)$ may in general be more efficient than the QMLE under unknown heteroskedasticity because of the optimal weighting.

\subsection{On the Inference of Elements in Impact Matrices}

Assessing the statistical significance of the effect of a change in a regressor on the dependent variable is one of the main objectives of applied economists. In spatial regressions, as shown in Section 2, one first has to compute the reduced form of the specification and calculate the matrix of partial derivatives of the dependent variable with respect to the concerned regressor in order to figure out the matrix of impacts. 
Inference regarding causal effects should then be based on this matrix, which, for regressor $X_{n k}$, is presented in (6). All the elements of this impact matrix are possibly different from each other and performing inference on them would be of value.

For the SAR model, LeSage and Pace (2009) propose a Bayesian Markov chain Monte Carlo approach to produce inference on the scalar summary of effects, namely the average direct, indirect and total impacts. In this paper, we take the classical approach based on the Delta method to perform inference on those elements of the impact matrix. Statistical significance on differences of impacts can also be assessed. For instance, one could be interested in testing if the effect of the $k$ th regressor for observation $i$ on $y_{n i}$ will be the same as of the $l$ th regressor (with $l$ possibly different from $k$ ) for individual $j$ on $y_{n j}$, with $j$ possibly different from $i$.

Let $\hat{\gamma}_{n}$ be a $\sqrt{n}$-consistent estimator of $\gamma$, and $e_{n i}$ be the $i$ th column of $I_{n}$. The impact of $x_{n, j p}(p$ th regressor for individual $j$ ) on $y_{n i}$ is estimated to be $e_{n i}^{\prime} e^{-\hat{\alpha}_{n} W_{n}} e_{n j} \hat{\beta}_{n p}$, and the effect of $x_{n, s q}$ on $y_{n r}$ is estimated to be $e_{n r}^{\prime} e^{-\hat{\alpha}_{n} W_{n}} e_{n s} \hat{\beta}_{n q}$. Then, by the mean value theorem,

$$
\begin{aligned}
& \sqrt{n}\left[\left(e_{n i}^{\prime} e^{-\hat{\alpha}_{n} W_{n}} e_{n j} \hat{\beta}_{n p}-e_{n r}^{\prime} e^{-\hat{\alpha}_{n} W_{n}} e_{n s} \hat{\beta}_{n q}\right)-\left(e_{n i}^{\prime} e^{-\alpha_{0} W_{n}} e_{n j} \beta_{0 p}-e_{n r}^{\prime} e^{-\alpha_{0} W_{n}} e_{n s} \beta_{0 q}\right)\right] \\
& =A_{1 n} \sqrt{n}\left(\hat{\alpha}_{n}-\alpha_{0}, \hat{\beta}_{n p}-\beta_{0 p}, \hat{\beta}_{n q}-\beta_{0 q}\right)^{\prime}+o_{P}(1) \\
& \stackrel{d}{\rightarrow} N\left(0, \lim _{n \rightarrow \infty} A_{1 n} B_{1 n} A_{1 n}^{\prime}\right),
\end{aligned}
$$

where $A_{1 n}=\left[-e_{n i}^{\prime} e^{-\alpha_{0} W_{n}} W_{n} e_{n j} \beta_{0 p}+e_{n r}^{\prime} e^{-\alpha_{0} W_{n}} W_{n} e_{n s} \beta_{0 q}, e_{n i}^{\prime} e^{-\alpha_{0} W_{n}} e_{n j},-e_{n r}^{\prime} e^{-\alpha_{0} W_{n}} e_{n s}\right]$ and $B_{1 n}$ is the asymptotic VC matrix of $\sqrt{n}\left(\hat{\alpha}_{n}-\alpha_{0}, \hat{\beta}_{n p}-\beta_{0 p}, \hat{\beta}_{n q}-\beta_{0 q}\right)^{\prime}$. To test whether the two impacts are equal, we may use the asymptotically standard normal statistic $\sqrt{n}\left(e_{n i}^{\prime} e^{-\hat{\alpha}_{n} W_{n}} e_{n j} \hat{\beta}_{n p}-e_{n r}^{\prime} e^{-\hat{\alpha}_{n} W_{n}} e_{n s} \hat{\beta}_{n q}\right) /\left(\hat{A}_{1 n} \hat{B}_{1 n} \hat{A}_{1 n}^{\prime}\right)^{1 / 2}$ under the null hypothesis, where $\hat{A}_{1 n}$ and $\hat{B}_{1 n}$ are, respectively, consistent estimates of $A_{1 n}$ and $B_{1 n}$. Another example is in testing whether the average direct effect $\frac{1}{n} \operatorname{tr}\left(e^{\hat{\alpha}_{n} W_{n}}\right) \hat{\beta}_{n p}$ is significantly different from zero. It can be shown that

$$
\begin{aligned}
\frac{1}{\sqrt{n}} \operatorname{tr}\left(e^{-\hat{\alpha}_{n} W_{n}}\right) \hat{\beta}_{n p}-\frac{1}{\sqrt{n}} \operatorname{tr}\left(e^{-\alpha_{0} W_{n}}\right) \beta_{0 p} & =A_{2 n} \sqrt{n}\left[\hat{\alpha}_{n}-\alpha_{0}, \hat{\beta}_{n p}-\beta_{0 p}\right]^{\prime}+o_{P}(1) \\
& \stackrel{d}{\rightarrow} N\left(0, \lim _{n \rightarrow \infty} A_{2 n} B_{2 n} A_{2 n}^{\prime}\right)
\end{aligned}
$$

where $A_{2 n}=\left[-\frac{1}{n} \operatorname{tr}\left(e^{-\alpha_{0} W_{n}} W_{n}\right) \beta_{0 p}, \frac{1}{n} \operatorname{tr}\left(e^{-\alpha_{0} W_{n}}\right)\right]$ and $B_{2 n}$ is the asymptotic VC matrix of $\sqrt{n}\left[\hat{\alpha}_{n}-\right.$ $\left.\alpha_{0}, \hat{\beta}_{n p}-\beta_{0 p}\right]^{\prime}$. Let $\hat{A}_{2 n}$ and $\hat{B}_{2 n}$ be, respectively, consistent estimates of $A_{2 n}$ and $B_{2 n}$.

Lemma 1. $\sqrt{n}\left[\left(e_{n i}^{\prime} e^{-\hat{\alpha}_{n} W_{n}} e_{n j} \hat{\beta}_{n p}-e_{n r}^{\prime} e^{-\hat{\alpha}_{n} W_{n}} e_{n s} \hat{\beta}_{n q}\right)-\left(e_{n i}^{\prime} e^{-\alpha_{0} W_{n}} e_{n j} \beta_{0 p}-e_{n r}^{\prime} e^{-\alpha_{0} W_{n}} e_{n s} \beta_{0 q}\right)\right]\left(\hat{A}_{1 n} \hat{B}_{1 n} \hat{A}_{1 n}^{\prime}\right)^{-1 / 2} \stackrel{d}{\rightarrow}$ $N(0,1)$ and $\frac{1}{\sqrt{n}}\left[\operatorname{tr}\left(e^{-\hat{\alpha}_{n} W_{n}}\right) \hat{\beta}_{n p}-\operatorname{tr}\left(e^{-\alpha_{0} W_{n}}\right) \beta_{0 p}\right]\left(\hat{A}_{2 n} \hat{B}_{2 n} \hat{A}_{2 n}^{\prime}\right)^{-1 / 2} \stackrel{d}{\rightarrow} N(0,1)$. 
Several applications of this lemma will be presented in Section 5 which is dedicated to the application of the MESS to figure out the dominant type of outward FDI for Belgium. However, before turning to the empirical application, we first present Monte Carlo experiments which assess the finite sample performance of the MLEs, QMLEs and GMMEs.

\section{Monte Carlo Simulations}

The considered model is presented in (42).

$$
e^{\alpha W_{n}} y_{n}=\beta_{1} l_{n}+\beta_{2} X_{n 1}+\beta_{3} X_{n 2}+u_{n}, \quad e^{\tau W_{n}} u_{n}=\epsilon_{n}
$$

The interaction matrix $W_{n}$ is defined as the 5 nearest neighbors ${ }^{21}, y_{n}$ is the $n \times 1$ vector of the dependent variable and $\alpha$ is the parameter capturing the intensity of interactions between observations. The first explanatory variable is a constant term, $X_{n 1}=\left[x_{n 1,1}, \ldots, x_{n 1, n}\right]^{\prime}$ is an independent standard normal vector while for the definition of $X_{n 2}$, we follow Pace et al. (2011) and generate a spatially autocorrelated regressor. Its specification is $X_{n 2}=\left(I-0.7 W_{n}\right)^{-1} v$, with $v$ a standard multivariate normal random vector of dimension $n$. In all the simulations, we keep the design space constant. Four different specifications for the error term are considered and presented in Table 1. We first assume an ideal world with an homoskedastic normally

Table 1: Specifications for the error term

\begin{tabular}{c|c|c|} 
& Homoskedasticity & Heteroskedasticity \\
\hline Normal & $\epsilon_{n i}=\nu_{n i}$, & $\epsilon_{n i}=\nu_{n i} \sqrt{z_{n i}}$, \\
& $\nu_{n i} \sim N\left(0, \sigma^{2}\right)$ & $\nu_{n i} \sim N\left(0, \sigma^{2}\right), z_{n i}=e^{X_{n 1, i}}+e^{X_{n 2, i}}$ \\
\hline Non Normal & $\epsilon_{n i}=\left(\frac{\nu_{n i}-3}{\sqrt{6}}\right) \sigma$, & $\epsilon_{n i}=\left(\frac{\nu_{n i}-3}{\sqrt{6}}\right) \sqrt{z_{n i}} \sigma$, \\
& $\nu_{n i} \sim \chi_{3}^{2}$ & $\nu_{n i} \sim \chi_{3}^{2}, z_{n i}=e^{X_{n 1, i}}+e^{X_{n 2, i}}$ \\
\hline
\end{tabular}

distributed error term (upper left panel of Table 1). The lower left panel still imposes homoskedasticity but replaces the normality by a $\chi_{3}^{2}$ distributed disturbance. The third case, presented in the upper right panel, assumes normal but heteroskedastic error term. Heteroskedasticity is assumed of exponential form, but in the simulations, we treat it as of unknown form. Finally, the last case relaxes both normality and homoskedasticity.

The value of $\sigma^{2}$ is set to keep the signal-to-noise ratio, $R^{2}$ in our notations, constant. This $R^{2}$ is defined

\footnotetext{
${ }^{21}$ The weight matrix has been normalized by the spectral radius. However, due to its special structure, row-normalization provides the same normalization.
} 
as the share of explained variance of $y$ in the total variance and its expression is presented in (43).

$$
R^{2}=\frac{\beta^{\prime}(X-\bar{X})^{\prime} e^{-\alpha W_{n}^{\prime}} e^{-\alpha W_{n}}(X-\bar{X}) \beta}{\beta^{\prime}(X-\bar{X})^{\prime} e^{-\alpha W_{n}^{\prime}} e^{-\alpha W_{n}}(X-\bar{X}) \beta+\sigma^{2} \operatorname{tr}\left(e^{-(\alpha+\tau) W_{n}^{\prime}} e^{-(\alpha+\tau) W_{n}}\right)}
$$

In these simulations, we maintain this signal-to-noise ratio equal to 0.8. Besides, three different sample sizes are considered, $n=254,589,975$, corresponding respectively to the number of counties in Texas, municipalities in Belgium, and the number of counties belonging to the South States of USA ${ }^{22}$. Finally, the values of $\alpha$ and $\tau$ vary from -1 to 1 by increment of 0.5 while $\beta_{1}, \beta_{2}$ and $\beta_{3}$ are all set to 1 . All the experiments were replicated 1000 times. For these simulations, the GMM estimator is computed as a two step feasible optimal GMM using the moment vector $\hat{g}_{n, d}^{\#}(\gamma)$ in $(39) .^{23}$

Tables $2-5$ summarize the results of the experiments. The sample has the moderate size of $n=254 .^{24}$ We report the bias, root mean squared error (RMSE) (in italics) and standard errors (in bold) for both QML and GMM estimators of $\alpha, \tau, \beta_{1}, \beta_{2}$ and $\beta_{3}$. We look at the results for the different sample sizes. Tables 2 and 3 summarize QMLE results while Tables 4 and 5 present the results of the GMME. The 2 left panels of each Table summarize output for the homoskedastic case while the two right panels are concerned with heteroskedastic disturbances. We first observe that results in terms of bias, standard error and RMSE are similar for QML and GMM estimators. Besides, the non-normality of the error term does not seem to affect the results. When the DGP includes heteroskedasticity, we observe an increase in the standard errors and RMSE of both estimators while their bias remains similar to the homoskedastic case. It is also worthwhile to note that the bias of both estimators is relatively small even though the bias of $\alpha$ is a bit smaller than that of $\tau$, the parameter capturing spatial autocorrelation in the errors. It also seems that the results are slightly better for $\beta_{2}$ and $\beta_{3}$ than for the constant, in terms of bias, standard errors and RMSE. Finally, we do not observe any difference in the simulations results regarding the spatially autocorrelated explanatory variable and the one directly drawn from a standard normal distribution.

\footnotetext{
${ }^{22}$ These States correspond to Alabama, Arizona, Arkansas, Florida, Georgia, Louisiana, Mississippi, New Mexico, North Carolina, Oklahoma, South Carolina, Tennessee and Texas.

${ }^{23}$ In the first step, the weighting matrix of the GMM is assumed to be the identity matrix.

${ }^{24}$ The results of the experiments for $n=589$ and $n=975$ are similar. We observe that bias, standard errors and thus RMSE are lower when the sample size becomes larger. These results are reported in the supplementary file.
} 


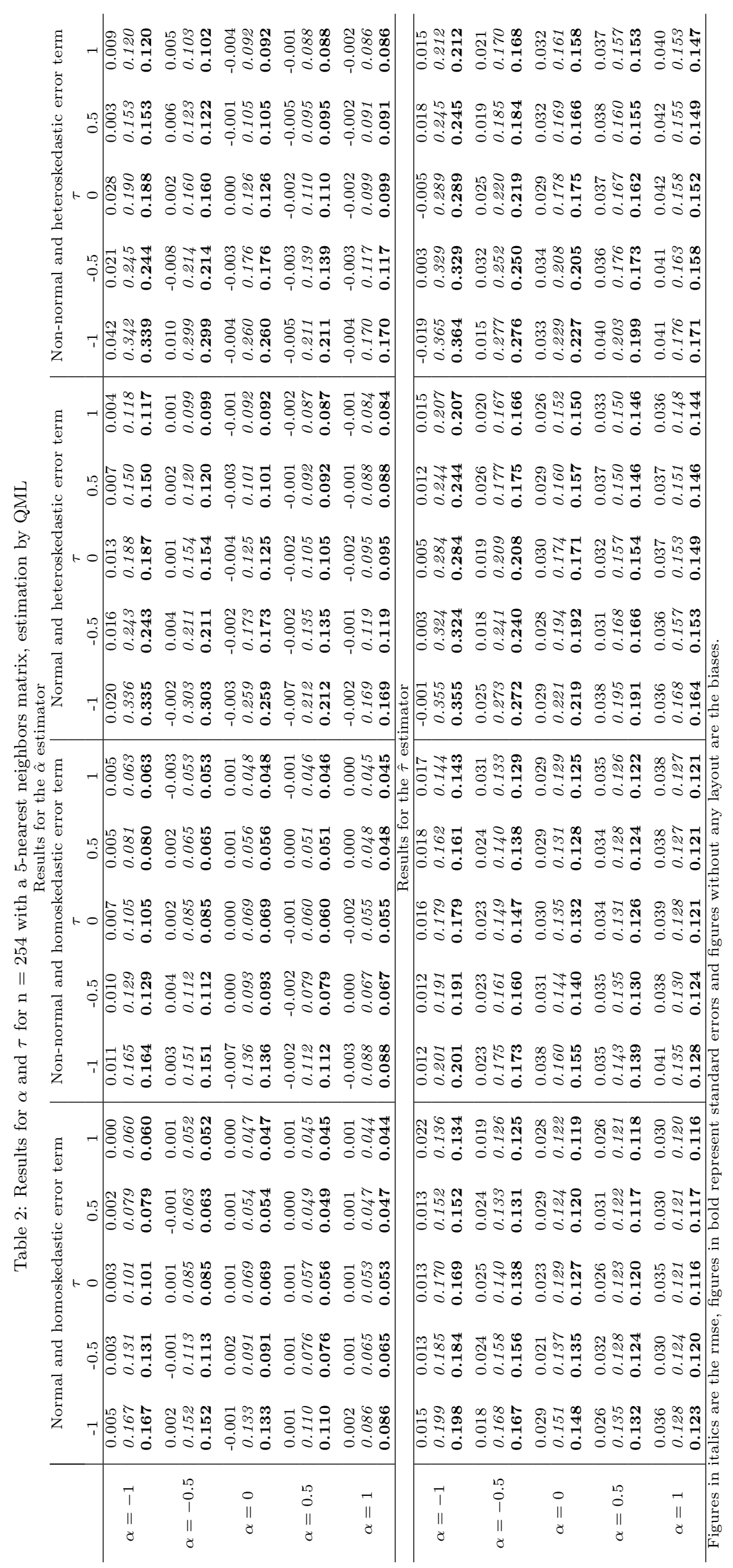




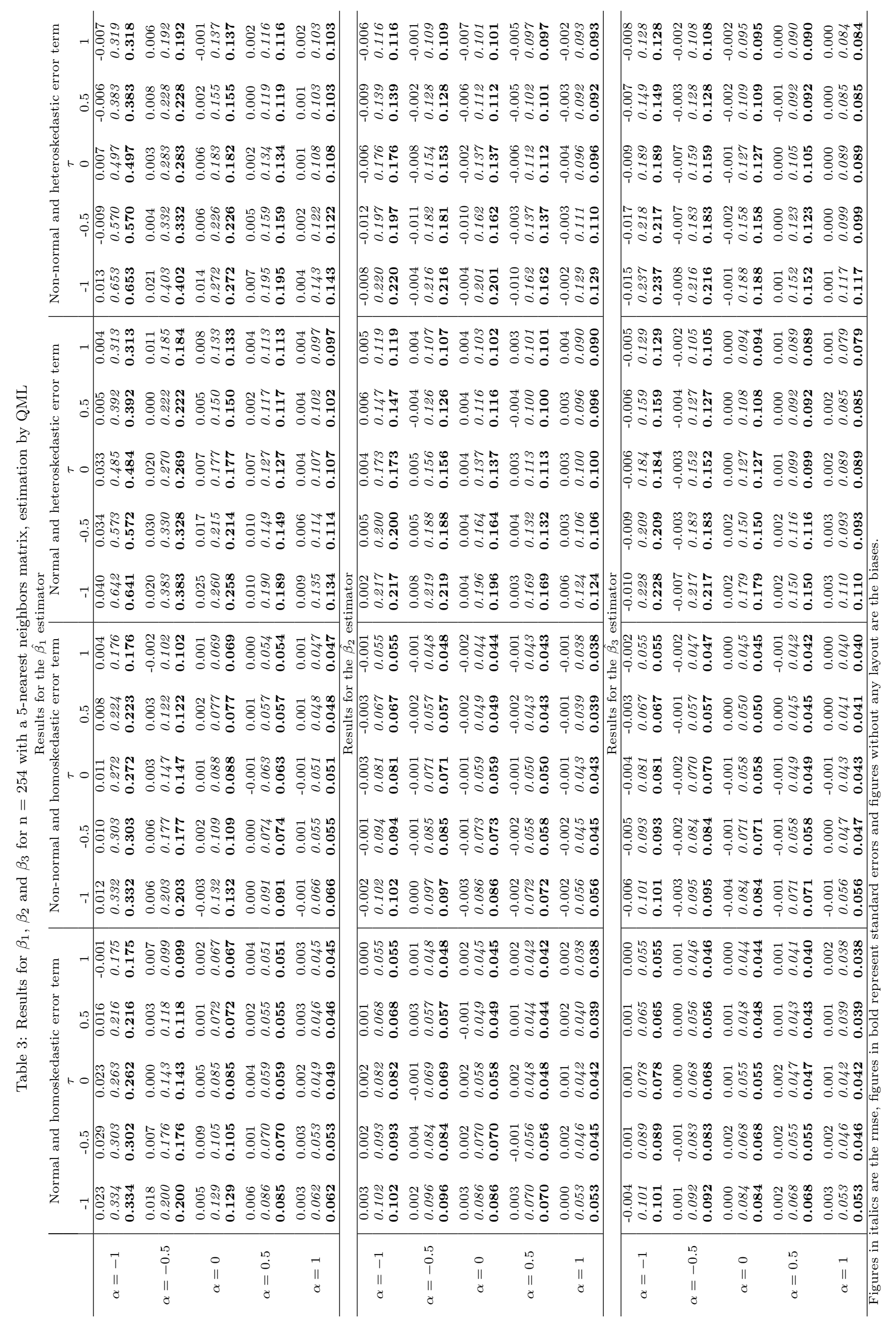




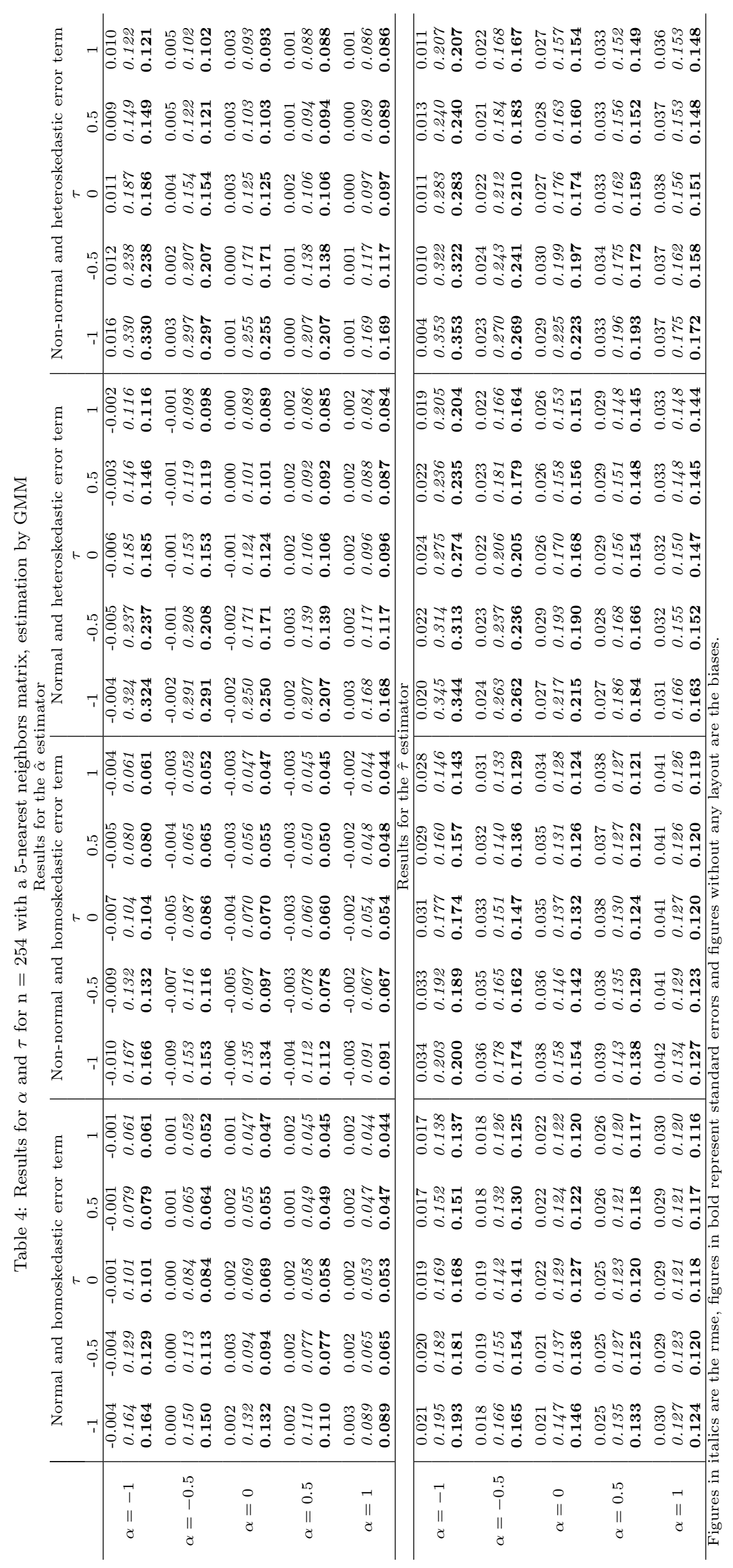




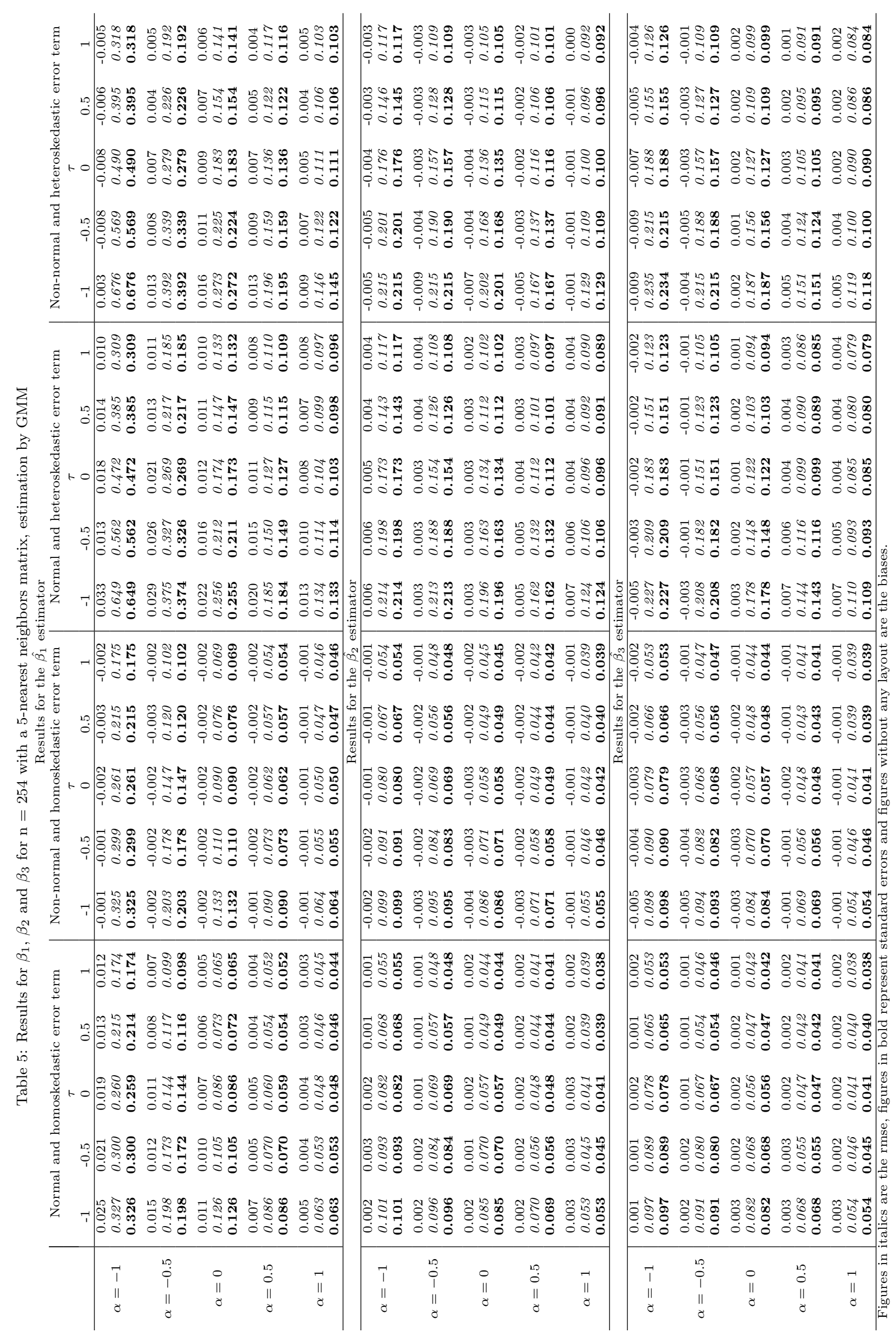




\section{Application to Belgium's outward FDI}

To the best of our knowledge, with the recent exceptions of Coughlin and Segev (2000); Blonigen et al. (2007); Baltagi et al. (2007, 2008) and Garretsen and Peeters (2009), the literature on FDI has overlooked the third country effect as determinant of bilateral FDI. Coughlin and Segev (2000) consider inward FDI for 29 Chinese provinces and found positive and significant spatially autocorrelated error terms (SEM specification). Blonigen et al. (2007) distinguish 4 different types of FDI that multinational enterprises (MNEs) can undertake. These 4 cases are summarized in Table 6 (corresponding to Table 1 in Blonigen et al., 2007) and can be identified based on the sign of the spatial lag parameter and of the surrounding-market potential variable. ${ }^{25}$ MNEs can firstly embark in FDI for market access reasons and avoidance of high trade or tariff

Table 6: Expected sign for spatial lag and surrounding-market potential variables

\begin{tabular}{lcc}
\hline FDI Motivation & Sign of spatial lag & $\begin{array}{c}\text { Sign of surrounding-market } \\
\text { potential variable }\end{array}$ \\
\hline Pure horizontal & 0 & 0 \\
Export-platform & - & + \\
Pure vertical & - & 0 \\
Vertical specialization & + & 0 \\
\hline Source: Blonigen et al. (2007) & &
\end{tabular}

costs in a host country. This is horizontal FDI. If trade barriers between the parent country (where the MNE is located) and host country (where the MNE would like to make its products available) are too high, the MNE could decide to build a plant in the latter country to avoid export costs but at the expense of building a new production plant. Blonigen et al. (2007) note that no spatial autocorrelation between FDI should be observed since MNEs make independent decisions about serving a market either through exports or affiliate sales. Besides, for this basic form of FDI, we do not expect any market potential effect of host country since the MNE looks for access to the considered market only.

A second motivation for FDI occurs if trade barriers between a set of destination markets are lower than trade frictions between these destination markets and the parent country. In that setup, a MNE could decide to build a plant in a host country, export to other markets and facing lower trade costs only. This type of FDI is called export-platform. As the MNE will not build a production plant in each host country, we expect a negative spatial autocorrelation between neighboring FDI locations. However, we anticipate a positive effect of the surrounding-market potential variable since the MNE will locate its new plant in the host country which has access to the largest surrounding market.

\footnotetext{
${ }^{25}$ Since the data we have do concern countries and not MNEs, we can only observe the dominant type of MNE behavior in terms of FDI, since the data may contain a mixture of the different motivations for FDI.
} 
MNEs will make vertical FDI if they want to access to cheaper factor inputs for their products. In its simplest form, namely pure vertical, host countries are in competition in terms of input factor prices to receive FDI. Hence, we expect a negative spatial autocorrelation between FDI. However, since the product is shipped back to the parent country to be further processed, not any effect from surrounding-market potential is foreseen. A more complex form of vertical FDI has been developed by Davies (2005) and Baltagi et al. (2007). Within that framework, named vertical specialization, the MNE decides to split its vertical chain of production among possibly several host countries, to benefit from the comparative advantage of the hosts. In such a framework, according to Blonigen et al. (2007), we should observe positive spatial autocorrelation due to possible agglomeration forces such as the presence of immobile resources, since the suppliers' presence in neighboring host countries is likely to increase FDI to a particular market. However, for the same reason as in pure vertical FDI, we do not predict any surrounding-market effect.

Blonigen et al. (2007) use outbound US FDI to 35 countries over the period 1983 to 1998 to test the dominant type of FDI which characterizes US MNEs. Even though they found a positive and significant effect of surrounding-market potential on their full sample, the authors acknowledge the fragility of their results with respect to the countries considered. Besides, they could not conclude to the presence of spatial autocorrelation for the full sample when fixed effects are included in the specification. Garretsen and Peeters (2009) also test the dominant motivation for FDI using outward Dutch FDI to 19 countries from 1984 to 2004. When analyzing their complete sample, they find a positive and significant market potential effect but also positive and significant spatial autocorrelation among FDI.

Our contribution to this literature is threefold. Firstly, we analyze the dominant pattern of Belgium's outward FDI using a modified gravity equation which, in addition to traditional determinants found in the literature, also captures effects of spatial interactions and market potential. We secondly compare results using a MESS(1,1) and a SARAR specification and highlight the similarities in terms of economic interpretations of these two models. We finally apply the lemma concerning inference to assess statistical significance of elements of impact matrices of FDI's determinants.

\subsection{Data and empirical specification}

This application concerns Belgium's outward FDI into 35 countries in 2009. These 35 host countries belong either to OECD or European Union and represent $94 \%$ of Belgium's total outward FDI. ${ }^{26}$

\footnotetext{
${ }^{26}$ The countries considered are: Australia, Austria, Bulgaria, Canada, Cyprus, Czech Republic, Denmark, Estonia, Finland, France, Germany, Greece, Hungary, Ireland, Italy, Japan, South Korea, Latvia, Lithuania, Luxembourg, Mexico, Netherlands, New Zealand, Norway, Poland, Portugal, Romania, Slovakia, Slovenia, Spain, Sweden, Switzerland, Turkey, United Kingdom and United States of America.
} 
The modified gravity to be estimated is presented in (44).

$$
L F D I_{i}=\beta_{1}+\beta_{2} L G D P_{i}+\beta_{3} L P O P_{i}+\beta_{4} O E C D_{i}+\beta_{5} L D I S_{i}+\beta_{6} \text { TARIFF }_{i}+\beta_{7} M_{i}+\epsilon_{i} .
$$

$L F D I_{i}$ is the stock of outward FDI (in logs) from Belgium to host country $i$. FDI stocks were extracted from the OECD International Direct Investment Statistics. The set of regressors includes host GDP in $\operatorname{logs}(L G D P)$, host population in logs $(L P O P)$, an OECD dummy which captures an OECD effect, the bilateral distance between Belgium and country $i$ expressed in logs $(L D I S)$ and a measure of trade costs which corresponds to the weighted mean of applied tariffs on all products, as defined by the World bank WDI database and labeled as TARIFFS. The last exogenous regressor is the surrounding-market potential variable, MP. We follow a similar approach to Blonigen et al. (2007) in the definition of this variable. For host country $i$, the market potential is computed as the sum of inverse-distance weighted log-GDPs of all other $k \neq i$ countries in the world for which we could obtain GDP data (this amounts to 183 countries). The only difference with Blonigen et al. (2007) comes from their use of the log of the inverse-distance weighted GDP instead. This difference in the position of the logarithm is motivated by the fact that as the host GDP enters in logs, we believe the surrounding market variable should also be based on logged GDP. ${ }^{27} L G D P$, $L P O P$ and TARIFFS all come from the World Bank WDI database while bilateral distances and distances used to construct the $M P$ variable come from CEPII's databases. Finally, all the concerned variables are expressed in constant USD of 2000. Some descriptive statistics of the data are presented in Table 7.

\begin{tabular}{c|cccc}
\multicolumn{5}{c}{ Table 7: Descriptive statistics for the data } \\
& Mean & Std dev & Min & Max \\
\hline LFDI & 8.3942 & 1.9970 & 4.4103 & 11.8510 \\
LGDP & 25.973 & 1.775 & 22.782 & 30.048 \\
LPOP & 16.390 & 1.450 & 13.118 & 19.542 \\
OECD & 0.857 & 0.355 & 0 & 1 \\
LDIS & 7.337 & 1.157 & 5.154 & 9.853 \\
TARIFFS & 1.877 & 1.369 & 0.990 & 8.930 \\
MP & 1.364 & 0.491 & 0.356 & 2.257 \\
\hline
\end{tabular}

Accounting for spatial autocorrelation in FDI requires the setup of an interaction scheme, modeled through the interaction (spatial weights) matrix $W_{n}$. In this application, we follow Blonigen et al. (2007) and use an inverse arc-distance between capitals to model interactions between host countries. However, we do not multiply the weights by the shortest distance between capitals as done in Blonigen et al. (2007)

\footnotetext{
${ }^{27}$ Garretsen and Peeters (2009) construct their surrounding-market potential variable in a different way since they only consider the GDP of all host countries in the sample.
} 
since we do not row-normalize our weight matrix but instead use the spectral radius to standardize the matrix. ${ }^{28}$ This approach is advocated by Baltagi et al. (2008) who argue that row-normalizing a distance based interaction matrix converts absolute distance-based interactions to relative distance-based and thus changes the information content of the interaction scheme. ${ }^{29}$ In addition, we control for the presence of residual spatial autocorrelation in the error terms. We have the same interaction matrix for both MESS processes. As shown in Section 3, the QMLE can be consistent in presence of unknown heteroskedasticity.

Table 8 summarizes the results of different econometric specifications which extend (44). Columns 2-8 present estimation results respectively for OLS (corrected for heteroskedasticity), homoskedastic SARAR (by QML), homoskedastic MESS(1,1) (by QML), homoskedastic MESS(1,1) (by optimal GMM with the moment vector $\hat{g}_{n, d}^{\#}(\gamma)$ in $(39)$ ), heteroskedastic SARAR (by optimal $\operatorname{GMM}^{30}$ ), heteroskedastic MESS $(1,1$ ) (by QML) and heteroskedastic $\operatorname{MESS}(1,1)$ (by optimal GMM with the moment vector $\hat{g}_{n, d}^{\#}(\gamma)$ in $(39)$ ).

The first result being worth highlighting concerns the bias of the OLS estimator due to omission of spatial autocorrelation. We observe a negative and significant (at the $10 \%$ threshold) estimated coefficient for the surrounding-market potential variable, $M P$, while this estimated coefficient becomes positive but looses its significance when spatial autocorrelation is accounted for. ${ }^{31}$ In terms of economic interpretation, least squares estimates would imply a negative elasticity of market potential on FDI, a case which does not fit in the considered economic theory. Besides, we also observe an estimated value of the OECD dummy twice smaller in the least squares regressions compared to spatial regressions and this variable becomes significant at the $10 \%$ level in the latter case, but we will come back on these interpretation later on. The parameter of the population variable is also affected since its estimated value by least squares $(-0.479)$ is on average around $25 \%$ higher than in spatial models. These results provide thus strong arguments to account for spatial autocorrelation in the specification. The rest of the discussion will thus focus on the 6 last columns of Table 8, which rely on spatial econometrics' methods.

Secondly, the quasi maximum likelihood and GMM estimation of the MESS(1,1) with homoskedastic and heteroskedastic disturbances provide similar results for both estimated values and standard errors.

The last result we would like to pinpoint relates to the sign of spatial autocorrelation. We observe a negative spatial autocorrelation for both SARAR specifications (homoskedastic and heteroskedastic) while

\footnotetext{
${ }^{28}$ As each weight will be multiplied by a common factor, the spectral radius will also be multiplied by this factor, implying that the normalized matrix will be the same, no matter if the interaction matrix is initially rescaled or not.

${ }^{29}$ For further information concerning matrix normalizations, interested readers may consult Kelejian and Prucha (2010).

${ }^{30}$ In the moment vector, the instruments for the linear moments are $\hat{R}_{n}\left[X_{n}, W_{n} \hat{S}_{n}^{-1} X_{n} \hat{\beta}_{n}\right]$ and the matrices for the quadratic moments are $\hat{R}_{n} W_{n} \hat{S}_{n}^{-1} \hat{R}_{n}^{-1}-\operatorname{Diag}\left(\hat{R}_{n} W_{n} \hat{S}_{n}^{-1} \hat{R}_{n}^{-1}\right)$ and $M_{n} \hat{R}_{n}^{-1}-\operatorname{Diag}\left(M_{n} \hat{R}_{n}^{-1}\right)$, where $\hat{R}_{n}=I_{n}-\hat{\rho}_{n} M_{n}, \hat{S}_{n}=I_{n}-\hat{\lambda}_{n} W_{n}$ and $\left(\hat{\lambda}_{n}, \hat{\rho}_{n}, \hat{\beta}_{n}^{\prime}\right)^{\prime}$ is an initial GMME.

${ }^{31} \mathrm{~A}$ more rigorous analysis, based on impacts of explanatory variables computed from the reduced form of the spatial regressions is presented later on.
} 
the MESS(1,1) provides a positive value for $\alpha$. Thus, a negative spatial autocorrelation translates in a positive $\alpha .{ }^{32}$ Finally, we observe that there is not any significant spatial autocorrelation left in the error terms.

The computation of matrices of impacts of changes in determinants on FDI is required to be able to give conclusions regarding the dominant type of FDI characterizing Belgium. Indeed, as MESS(1,1) and SARAR are estimated under implicit form (see (2) and (3)), we need to compute their associated reduced form and then calculate the matrix of partial derivatives with respect to each explanatory variable to get impact matrices. For the $\operatorname{MESS}(1,1)$, this impact matrix for regressor $X_{n k}$ is shown in (6).

\begin{tabular}{c|ccccccc}
\multicolumn{7}{c}{ Table 8: Estimation results for different specifications } \\
& $(1)$ & $(2)$ & $(3)$ & $(4)$ & $(5)$ & $(6)$ & $(7)$ \\
\hline Cons. & -1.745 & -1.664 & -3.021 & -3.159 & -1.266 & -3.023 & -2.989 \\
& $(4.668)$ & $(4.628)$ & $(4.660)$ & $(4.671)$ & $(3.899)$ & $(4.288)$ & $(4.291)$ \\
$L G D P$ & $1.038^{* * *}$ & $1.093^{* * *}$ & $1.114^{* * *}$ & $1.121^{* * *}$ & $1.089^{* * *}$ & $1.113^{* * *}$ & $1.113^{* * *}$ \\
& $(0.265)$ & $(0.237)$ & $(0.246)$ & $(0.246)$ & $(0.220)$ & $(0.241)$ & $(0.241)$ \\
LPOP & $-0.479^{*}$ & $-0.584^{* *}$ & $-0.585^{* *}$ & $-0.595^{* *}$ & $-0.591^{* *}$ & $-0.584^{* *}$ & $-0.584^{* *}$ \\
& $(0.268)$ & $(0.239)$ & $(0.246)$ & $(0.246)$ & $(0.235)$ & $(0.254)$ & $(0.254)$ \\
OECD & 0.542 & $1.024^{*}$ & $1.037^{*}$ & $1.081^{*}$ & $1.023^{*}$ & $1.037^{*}$ & $1.033^{*}$ \\
& $(0.702)$ & $(0.544)$ & $(0.551)$ & $(0.553)$ & $(0.605)$ & $(0.611)$ & $(0.611)$ \\
LDIS & $-1.162^{* * *}$ & $-1.271^{* * *}$ & $-1.199^{* * *}$ & $-1.201^{* * *}$ & $-1.293^{* * *}$ & $-1.199^{* * *}$ & $-1.200^{* * *}$ \\
& $(0.217)$ & $(0.237)$ & $(0.220)$ & $(0.220)$ & $(0.209)$ & $(0.200)$ & $(0.200)$ \\
TARIFFS & 0.089 & 0.104 & 0.106 & 0.108 & 0.108 & 0.106 & 0.106 \\
& $(0.078)$ & $(0.112)$ & $(0.113)$ & $(0.113)$ & $(0.084)$ & $(0.084)$ & $(0.084)$ \\
MP & $-0.786^{*}$ & 1.123 & 1.212 & 1.394 & 1.156 & 1.212 & 1.196 \\
& $(0.407)$ & $(1.090)$ & $(1.105)$ & $(1.128)$ & $(1.147)$ & $(1.186)$ & $(1.185)$ \\
Spat auto & - & $-0.326^{* *}$ & $0.265^{* *}$ & $0.286^{* * *}$ & $-0.335^{*}$ & $0.265^{* *}$ & $0.264^{* *}$ \\
in $y$ & & $(0.157)$ & $(0.109)$ & $(0.110)$ & $(0.173)$ & $(0.121)$ & $(0.121)$ \\
Spat auto & - & 0.275 & -0.004 & -0.010 & 0.282 & -0.004 & 0.001 \\
in errors & & $(0.439)$ & $(0.516)$ & $(0.516)$ & $(0.598)$ & $(0.419)$ & $(0.419)$ \\
\hline$n$ & 35 & 35 & 35 & 35 & 35 & 35 & 35
\end{tabular}

Standard errors between brackets; (1) is OLS (with White correction), (2) is homoskedastic SARAR, (3) is homo. MESS(1,1) by QML, (4) is homo. MESS(1,1) by GMM, (5) is heteroskedastic SARAR, (6) is hetero. MESS $(1,1)$ by QML and $(7)$ is hetero. MESS $(1,1)$ by GMM; ${ }^{*},{ }^{*}$ and *** correspond to significance at the $10 \%, 5 \%$ and $1 \%$ respectively.

To compare MESS(1,1) and SARAR results, we report in Table 9 the average direct effect and the total effect for each of the explanatory variables for heteroskedastic SARAR and MESS(1,1), estimated both by QML and GMM. The average direct effect is computed as the average of diagonal elements of the impact matrix $\frac{1}{n} \operatorname{tr}\left(\Xi_{X_{n k}}^{y_{n}}\right)$ while the total effect is defined as the sum of all of its elements $l_{n}^{\prime} \Xi_{X_{n k}}^{y_{n}} l_{n}$. In terms of impacts on the dependent variable, the main focus for economists, we observe a strong similarity of impacts

\footnotetext{
${ }^{32}$ This difference comes from the definition of these two models, as shown in Section 2.
} 
produced by the two specifications even though parameters capturing spatial autocorrelation are completely different from each other.

\begin{tabular}{c|ccc|ccc} 
& \multicolumn{5}{|c}{ Table 9: Comparison of average direct effects and total effects } \\
& Direct effects & & Total effects \\
& SARAR & MESS(1,1) & MESS(1,1) & SARAR & MESS(1,1) & MESS(1,1) \\
& & QML & GMM & & QML & GMM \\
\hline LGDP & 1.094 & 1.116 & 1.115 & 30.936 & 32.243 & 32.249 \\
LPOP & -0.595 & -0.586 & -0.585 & -16.802 & -16.926 & -16.926 \\
OECD & 1.029 & 1.039 & 1.035 & 29.085 & 30.025 & 29.938 \\
LDIS & -1.300 & -1.202 & -1.202 & -36.749 & -34.720 & -34.775 \\
TARIFFS & 0.109 & 0.107 & 0.106 & 3.070 & 3.076 & 3.074 \\
M_PO & 1.163 & 1.215 & 1.198 & 32.854 & 35.094 & 34.659 \\
\hline
\end{tabular}

Effects are computed from estimation results of heteroskedastic SARAR and $\operatorname{MESS}(1,1)$ (estimated by QML and GMM)

The lemma derived in Section 3 allows performing inference on elements of the impact matrices of the MESS(1,1). Table 10 summarizes inference results performed on different (functions of) elements of these impact matrices, based on the heteroskedastic MESS $(1,1)$ estimated by GMM. The first row analyzes the significance of average direct effects. The results indicate a non-significant elasticity of surrounding-market potential on FDI. This result, combined with a negative spatial autocorrelation, points to the dominance of pure vertical type of FDI. To the best of our knowledge, this application is the first to indicate such a clear cut result. One possible explanation of this result lies in the production costs faced by Belgian multinationals in Belgium. Indeed, labor costs in Belgium are amongst the highest in Europe. ${ }^{33}$ Besides, determinants of the traditional gravity equation have the expected sign. We observe a positive and significant elasticity of GDP, which captures the wealth effect, while elasticities of population and bilateral distance are found to be negative. The OECD dummy is found to be significant at the $10 \%$ level while least squares estimated value was found to be non-significant. Finally, the tariffs variable is found to be non-significant which can be explained by the homogeneity of the sample.

The second row presents inference on the indirect effect of Austria on Slovakia, $\left(\Xi_{X_{n k}}^{y_{n}}\right)_{S V K, A U T}$. In other words, we analyze if a shock on a regressor in Austria will affect outward FDI from Belgium to Slovakia. We observe a significant effect for the host GDP variable and bilateral distance but the effect is non-significant for the four other regressors. For instance, increasing the GDP of Austria by 1\% will reduce outward FDI from Belgium to Slovakia by $0.147 \%$. Finally, the last row of Table 10 studies significance of the difference between the indirect effect of Mexico on the United-States and the indirect effect of Canada on United-

\footnotetext{
${ }^{33}$ See Eurostat database on labor costs.
} 
States , $\left(\Xi_{X_{n k}}^{y_{n}}\right)_{U S A, M E X}-\left(\Xi_{X_{n k}}^{y_{n}}\right)_{U S A, C A N}$. We observe significant difference between those indirect effects for GDP and bilateral distance. In other words, the effect of a variation of Mexican GDP on outward FDI from Belgium to the United States will be statistically different from the effect of the same variation of Canadian GDP on outward FDI from Belgium to the United States.

\begin{tabular}{c|ccccccc}
\multicolumn{1}{c}{ Table 10: Inference on elements of impact matrices } & \multicolumn{1}{c}{ or } \\
& LGDP & LPOP & OECD & LDIS & TARIFF & MP \\
\hline \multirow{2}{*}{$\operatorname{tr}\left(\Xi_{X_{n k}}^{y_{n}}\right)$} & $1.115^{* * *}$ & $-0.585^{* *}$ & $1.035^{*}$ & $-1.202^{* * *}$ & 0.106 & 1.198 \\
& $(0.242)$ & $(0.255)$ & $(0.613)$ & $(0.200)$ & $(0.084)$ & $(1.183)$ \\
$\left(\Xi_{X_{n k}}^{y_{n}}\right)_{S V K, A U T}$ & $-0.147^{*}$ & 0.077 & -0.137 & $0.159^{* *}$ & -0.014 & -0.158 \\
& $(0.084)$ & $(0.058)$ & $(0.120)$ & $(0.075)$ & $(0.013)$ & $(0.226)$ \\
$\left(\Xi_{X_{n k}}^{y_{n}}\right)_{U S A, M E X}-\left(\Xi_{X_{n k}}^{y_{n}}\right)_{U S A, C A N}$ & $0.009^{*}$ & -0.005 & 0.008 & $-0.010^{* *}$ & 0.001 & 0.010 \\
\hline
\end{tabular}

Standard errors are between brackets; AUT stands for Austria, CAN for Canada, MEX for Mexico, SVK for Slovakia and USA for the United States; $\frac{1}{n} \operatorname{tr}\left(\Xi_{X_{n k}}^{y_{n}}\right)$ is the average direct effect, $\left(\Xi_{X_{n k}}^{y_{n}}\right)_{S V K, A U T}$ is the indirect effect between Austria and Slovakia; $\left(\Xi_{X_{n k}}^{y_{n}}\right)_{U S A, M E X}-\left(\Xi_{X_{n k}}^{y_{n}}\right)_{U S A, C A N}$ is the difference between the indirect effect of a change in $x$ in Mexico on outward FDI in the United States and the indirect effect of a change in $x$ in Canada on outward FDI in the United States; ${ }^{*},{ }^{* *}$ and ${ }^{* * *}$ correspond to significance at the $10 \%, 5 \%$ and $1 \%$ respectively.

To conclude this section, even though SARAR and $\operatorname{MESS}(1,1)$ provide similar impacts, it is important to remark that the MESS is much easier to compute since there is no Jacobian of the transformation involved and the parameter space for $\alpha$ is not constrained.

\section{Conclusions}

This paper firstly develops the asymptotic theory of the matrix exponential spatial specification (MESS) in both the dependent variable and error terms. We show that the GMME is consistent and asymptotically normal even in the presence of unknown heteroskedasticity as long as the interaction matrix has zero diagonal elements. Besides we show that if the interaction matrices for the dependent variable and the error terms commute, the QMLE may also be consistent and asymptotically normal in the presence of unknown heteroskedasticity. In the homoskedastic case, we develop a best optimal GMME which is much simpler than the best optimal GMME for the SAR specification since moment conditions do not depend on estimated parameters. In case of non-normality, the homoskedastic best optimal GMME is shown to be more efficient than the QMLE. In the heteroskedastic case, a best optimal GMME cannot be derived except if we know the structure of heteroskedasticity. We thus develop an optimal GMME which is shown to be more efficient than the QMLE. We also derive a lemma to perform inference on the elements, or functions of them, of the impact matrices implied by the reduced form of the MESS, which is very important for applied 
economists. Monte Carlo experiments are conducted and show the good small sample properties of the proposed estimators. Finally, we apply our estimators to show that outward FDI from Belgium are mainly characterized by the vertical type. Results indicate that least squares estimation results are misleading. We also compare SARAR and MESS(1,1) impacts and note that they are very similar, which pleads for the use of the latter. When the spatial process is stable, the MESS has many advantages over the SAR model.

\section{Appendix A. QML Estimation of a high order MESS [MESS(p,q)]}

Consider the following high order MESS:

$$
e^{\alpha \mathbf{W}_{n}} y_{n}=X_{n} \beta+u_{n}, \quad e^{\tau \mathbf{M}_{n}} u_{n}=\epsilon_{n}, \quad \epsilon_{n}=\left(\epsilon_{n 1}, \ldots, \epsilon_{n n}\right)^{\prime},
$$

where $\boldsymbol{\alpha} \mathbf{W}_{n}$ denotes $\alpha_{1} W_{n 1}+\cdots+\alpha_{p} W_{n p}$ for a vector $\boldsymbol{\alpha}=\left(\alpha_{1}, \ldots, \alpha_{p}\right)^{\prime}$ and $n \times n$ spatial weights matrices $W_{n i}$ 's, $i=1, \ldots, p$, and $\boldsymbol{\tau} \mathbf{M}_{n}$ denotes $\tau_{1} M_{n 1}+\cdots+\tau_{q} M_{n q}$ for $\boldsymbol{\tau}=\left(\tau_{1}, \ldots, \tau_{q}\right)^{\prime}$ and $n \times n$ spatial weights matrices $M_{n j}$ 's, $j=1, \ldots, q$. Denote the model as MESS(p,q). We investigate the properties of the QMLE for this model when the disturbances are i.i.d. as assumed in Assumption 5. The quasi log likelihood function of the $\operatorname{MESS}(\mathrm{p}, \mathrm{q})$, as if the $\epsilon_{n i}$ 's were i.i.d. normal, is

$$
L_{n}(\boldsymbol{\theta})=-\frac{n}{2} \ln \left(2 \pi \sigma^{2}\right)+\ln \left|e^{\boldsymbol{\alpha} \mathbf{W}_{n}}\right|+\ln \left|e^{\tau \mathbf{M}_{n}}\right|-\frac{1}{2 \sigma^{2}}\left(e^{\boldsymbol{\alpha} \mathbf{W}_{n}} y_{n}-X_{n} \beta\right)^{\prime} e^{\left(\tau \mathbf{M}_{n}\right)^{\prime}} e^{\tau \mathbf{M}_{n}}\left(e^{\boldsymbol{\alpha} \mathbf{W}_{n}} y_{n}-X_{n} \beta\right),
$$

where $\theta=\left(\boldsymbol{\gamma}^{\prime}, \sigma^{2}\right)^{\prime}$ with $\boldsymbol{\gamma}=\left(\boldsymbol{\alpha}, \boldsymbol{\tau}, \beta^{\prime}\right)^{\prime}$. Let $\boldsymbol{\theta}_{0}$ be the true parameter vector. Since $\left|e^{\boldsymbol{\alpha} \mathbf{W}_{n}}\right|=e^{\operatorname{tr}\left(\boldsymbol{\alpha} \mathbf{W}_{n}\right)}$ and $\left|e^{\tau \mathbf{M}_{n}}\right|=e^{\operatorname{tr}\left(\boldsymbol{\tau} \mathbf{M}_{n}\right)}$, as long as $W_{n i}$ 's and $M_{n j}$ 's have zero diagonals, the log Jacobians disappear and the quasi $\log$ likelihood function is simplified to

$$
L_{n}(\boldsymbol{\theta})=-\frac{n}{2} \ln \left(2 \pi \sigma^{2}\right)-\frac{1}{2 \sigma^{2}}\left(e^{\boldsymbol{\alpha} \mathbf{W}_{n}} y_{n}-X_{n} \beta\right)^{\prime} e^{\left(\boldsymbol{\tau} \mathbf{M}_{n}\right)^{\prime}} e^{\boldsymbol{\tau} \mathbf{M}_{n}}\left(e^{\boldsymbol{\alpha} \mathbf{W}_{n}} y_{n}-X_{n} \beta\right)
$$

By contrast, for the high order SARAR model corresponding to (A.1),

$$
\left(I_{n}-\lambda \mathbf{W}_{n}\right) y_{n}=X_{n} \beta+u_{n}, \quad\left(I_{n}-\boldsymbol{\rho} \mathbf{M}_{n}\right) u_{n}=\epsilon_{n}, \quad \epsilon_{n}=\left(\epsilon_{n 1}, \ldots, \epsilon_{n n}\right)^{\prime}
$$

where $\boldsymbol{\lambda}=\left(\lambda_{1}, \ldots, \lambda_{p}\right)^{\prime}$ and $\boldsymbol{\rho}=\left(\rho_{1}, \ldots, \rho_{q}\right)^{\prime}$, the quasi $\log$ likelihood function involves the log Jacobian $\ln \left|\left(I_{n}-\boldsymbol{\lambda} \mathbf{W}_{n}\right)\left(I_{n}-\boldsymbol{\rho} \mathbf{M}_{n}\right)\right|=\ln \left|I_{n}-\boldsymbol{\lambda} \mathbf{W}_{n}\right|+\ln \left|I_{n}-\boldsymbol{\rho} \mathbf{M}_{n}\right|$. The stationary regions of $\boldsymbol{\lambda}$ and $\boldsymbol{\rho}$ can be hard to find and the Jacobian is computationally intensive (Elhorst et al., 2012). 
The MESS(p,q) (A.1) with the notations $\boldsymbol{\alpha} \mathbf{W}_{n}$ and $\boldsymbol{\tau} \mathbf{M}_{n}$ resembles the $\operatorname{MESS}(1,1)$ presented in (2), thus we have similar expressions for the QMLE. From (A.2), the QMLE of $\gamma$ is the minimizer of

$$
Q_{n}(\gamma)=\left(e^{\boldsymbol{\alpha} \mathbf{W}_{n}} y_{n}-X_{n} \beta\right)^{\prime} e^{\left(\tau \mathbf{M}_{n}\right)^{\prime}} e^{\tau \mathbf{M}_{n}}\left(e^{\boldsymbol{\alpha} \mathbf{W}_{n}} y_{n}-X_{n} \beta\right)
$$

For fixed $\boldsymbol{\phi}=\left(\boldsymbol{\alpha}^{\prime}, \boldsymbol{\tau}^{\prime}\right)^{\prime}$, the QMLE of $\beta$ is

$$
\hat{\beta}_{n}(\phi)=\left(X_{n}^{\prime} e^{\left(\tau \mathbf{M}_{n}\right)^{\prime}} e^{\tau \mathbf{M}_{n}} X_{n}\right)^{-1} X_{n}^{\prime} e^{\left(\tau \mathbf{M}_{n}\right)^{\prime}} e^{\tau \mathbf{M}_{n}} e^{\alpha \mathbf{W}_{n}} y_{n}
$$

Substituting $\hat{\beta}_{n}(\phi)$ into $Q_{n}(\gamma)$, we obtain a function of only $\phi$ :

$$
Q_{n}(\phi)=y_{n}^{\prime} e^{\left(\boldsymbol{\alpha} \mathbf{W}_{n}\right)^{\prime}} e^{\left(\tau \mathbf{M}_{n}\right)^{\prime}} H_{n}(\boldsymbol{\tau}) e^{\tau \mathbf{M}_{n}} e^{\boldsymbol{\alpha} \mathbf{W}_{n}} y_{n}
$$

where the projection matrix $H_{n}(\boldsymbol{\tau})=I_{n}-e^{\tau \mathbf{M}_{n}} X_{n}\left(X_{n}^{\prime} e^{\left(\tau \mathbf{M}_{n}\right)^{\prime}} e^{\tau \mathbf{M}_{n}} X_{n}\right)^{-1} X_{n}^{\prime} e^{\left(\tau \mathbf{M}_{n}\right)^{\prime}}$. The QMLE of $\boldsymbol{\phi}$ can be derived by the minimization of $Q_{n}(\phi)$. Corresponding to Assumptions 1, 3 and 4 , we make the following assumptions.

Assumption A.1. Matrices $\left\{W_{n i}\right\}$ for $i=1, \ldots, p$ and $\left\{M_{n j}\right\}$ for $j=1, \ldots, q$ are bounded in both row and column sum norms. The diagonal elements of $W_{n i}$ 's and $M_{n j}$ 's are zero.

Assumption A.2. There exists a constant $\delta>0$ such that $\left|\alpha_{i}\right| \leq \delta$ for $i=1, \ldots, p,\left|\tau_{j}\right| \leq \delta$ for $j=1, \ldots, q$, and the true $\boldsymbol{\phi}_{0}$ is in the interior of the parameter space $\mathbf{\Phi}=[-\delta, \delta]^{p+q}$.

Assumption A.3. The limit $\lim _{n \rightarrow \infty} \frac{1}{n} X_{n}^{\prime} e^{\left(\tau \mathbf{M}_{n}\right)^{\prime}} e^{\tau \mathbf{M}_{n}} X_{n}$ exists and is nonsingular for any $\boldsymbol{\tau} \in[-\delta, \delta]^{q}$, and the sequence of the smallest eigenvalues of $e^{\left(\boldsymbol{\tau} \mathbf{M}_{n}\right)^{\prime}} e^{\tau \mathbf{M}_{n}}$ is bounded away from zero uniformly in $\boldsymbol{\tau} \in$ $[-\delta, \delta]^{q}$.

To find the identification condition for $\phi$, define

$$
\begin{aligned}
\bar{Q}_{n}(\boldsymbol{\phi})= & \min _{\beta} \mathrm{E} Q_{n}(\boldsymbol{\gamma})=\left(X_{n} \beta_{0}\right)^{\prime} e^{-\left(\boldsymbol{\alpha}_{0} \mathbf{W}_{n}\right)^{\prime}} e^{\left(\boldsymbol{\alpha} \mathbf{W}_{n}\right)^{\prime}} e^{\left(\tau \mathbf{M}_{n}\right)^{\prime}} H_{n}(\boldsymbol{\tau}) e^{\tau \mathbf{M}_{n}} e^{\boldsymbol{\alpha} \mathbf{W}_{n}} e^{-\boldsymbol{\alpha}_{0} \mathbf{W}_{n}} X_{n} \beta_{0} \\
& +\sigma_{0}^{2} \operatorname{tr}\left(e^{-\left(\boldsymbol{\tau}_{0} \mathbf{M}_{n}\right)^{\prime}} e^{-\left(\boldsymbol{\alpha}_{0} \mathbf{W}_{n}\right)^{\prime}} e^{\left(\boldsymbol{\alpha} \mathbf{W}_{n}\right)^{\prime}} e^{\left(\tau \mathbf{M}_{n}\right)^{\prime}} e^{\tau \mathbf{M}_{n}} e^{\boldsymbol{\alpha} \mathbf{W}_{n}} e^{-\boldsymbol{\alpha}_{0} \mathbf{W}_{n}} e^{-\tau_{0} \mathbf{M}_{n}}\right) .
\end{aligned}
$$

The following condition is assumed for the identification uniqueness.

Assumption A.4. Either (i) $\lim _{n \rightarrow \infty} n^{-1}\left(X_{n} \beta_{0}\right)^{\prime} e^{-\left(\boldsymbol{\alpha}_{0} \mathbf{W}_{n}\right)^{\prime}} e^{\left(\boldsymbol{\alpha} \mathbf{W}_{n}\right)^{\prime}} e^{\left(\tau \mathbf{M}_{n}\right)^{\prime}} H_{n}(\boldsymbol{\tau}) e^{\tau \mathbf{M}_{n}} e^{\boldsymbol{\alpha} \mathbf{W}_{n}} e^{-\boldsymbol{\alpha}_{0} \mathbf{W}_{n}} X_{n} \beta_{0} \neq$ 0 for any $\boldsymbol{\tau}$ and $\boldsymbol{\alpha} \neq \boldsymbol{\alpha}_{0}$, and $\lim _{n \rightarrow \infty} n^{-1} \operatorname{tr}\left(e^{-\left(\boldsymbol{\tau}_{0} \mathbf{M}_{n}\right)^{\prime}} e^{\left(\boldsymbol{\tau} \mathbf{M}_{n}\right)^{\prime}} e^{\boldsymbol{\tau} \mathbf{M}_{n}} e^{-\boldsymbol{\tau}_{0} \mathbf{M}_{n}}\right)>1$ for any $\boldsymbol{\tau} \neq \boldsymbol{\tau}_{0}$, or (ii) $\lim _{n \rightarrow \infty} n^{-1} \operatorname{tr}\left(e^{-\left(\tau_{0} \mathbf{M}_{n}\right)^{\prime}} e^{-\left(\boldsymbol{\alpha}_{0} \mathbf{W}_{n}\right)^{\prime}} e^{\left(\boldsymbol{\alpha} \mathbf{W}_{n}\right)^{\prime}} e^{\left(\tau \mathbf{M}_{n}\right)^{\prime}} e^{\tau \mathbf{M}_{n}} e^{\boldsymbol{\alpha} \mathbf{W}_{n}} e^{-\boldsymbol{\alpha}_{0} \mathbf{W}_{n}} e^{-\tau_{0} \mathbf{M}_{n}}\right)>1$ for any $\boldsymbol{\phi} \neq \boldsymbol{\phi}_{0}$. 
The consistency of the QMLE follows from the uniform convergence of $\left[Q_{n}(\phi)-\bar{Q}_{n}(\phi)\right] / n$ to zero on the parameter space $\boldsymbol{\Phi}$ and the identification uniqueness. The proof of the following proposition is in Appendix C.

Proposition A.1. Under Assumptions 2, 5 and A.1-A.4, the $Q M L E \hat{\gamma}_{n}$ of the MESS(p,q) in (A.1) is consistent.

For the asymptotic distribution of $\hat{\gamma}_{n}$, a Taylor expansion of the first-order condition $\frac{\partial Q_{n}\left(\hat{\gamma}_{n}\right)}{\partial \gamma}=0$ at the true $\gamma_{0}$ yields

$$
\sqrt{n}\left(\hat{\gamma}_{n}-\gamma_{0}\right)=-\left(\frac{1}{n} \frac{\partial^{2} Q_{n}\left(\tilde{\gamma}_{n}\right)}{\partial \gamma \partial \gamma^{\prime}}\right)^{-1} \frac{1}{\sqrt{n}} \frac{\partial Q_{n}\left(\gamma_{0}\right)}{\partial \gamma}
$$

where $\tilde{\gamma}_{n}$ is between $\hat{\gamma}_{n}$ and $\gamma_{0}$. Under regularity conditions, $\frac{1}{n} \frac{\partial^{2} Q_{n}\left(\tilde{\boldsymbol{\gamma}}_{n}\right)}{\partial \boldsymbol{\gamma} \partial \boldsymbol{\gamma}^{\prime}}=\mathbf{C}_{n}+o_{P}(1)$ with $\mathbf{C}_{n}=$ $\frac{1}{n} \mathrm{E} \frac{\partial^{2} Q_{n}\left(\boldsymbol{\gamma}_{0}\right)}{\partial \boldsymbol{\gamma} \partial \boldsymbol{\gamma}^{\prime}}$. We assume that $\mathbf{C}_{n}$ is nonsingular in the limit.

Assumption A.5. The limit of $\mathbf{C}_{n}$ exists and is nonsingular.

The first-order derivatives of $Q_{n}(\gamma)$ at $\gamma_{0}$ are

$$
\begin{aligned}
& \frac{\partial Q_{n}\left(\boldsymbol{\gamma}_{0}\right)}{\partial \alpha_{i}}=2\left(X_{n} \beta_{0}+e^{-\boldsymbol{\tau}_{0} \mathbf{M}_{n}} \epsilon_{n}\right)^{\prime} e^{-\left(\boldsymbol{\alpha}_{0} \mathbf{W}_{n}\right)^{\prime}} \frac{\partial e^{\left(\boldsymbol{\alpha}_{0} \mathbf{W}_{n}\right)^{\prime}}}{\partial \alpha_{i}} e^{\left(\boldsymbol{\tau}_{0} \mathbf{M}_{n}\right)^{\prime}} \epsilon_{n}, \quad, i=1, \ldots, p, \\
& \frac{\partial Q_{n}\left(\boldsymbol{\gamma}_{0}\right)}{\partial \tau_{i}}=2 \epsilon_{n}^{\prime} e^{-\left(\boldsymbol{\tau}_{0} \mathbf{M}_{n}\right)^{\prime}} \frac{\partial e^{\left(\boldsymbol{\tau}_{0} \mathbf{M}_{n}\right)^{\prime}}}{\partial \tau_{i}} \epsilon_{n}, \quad i=1, \ldots, q, \\
& \frac{\partial Q_{n}\left(\boldsymbol{\gamma}_{0}\right)}{\partial \beta}=-2 X_{n}^{\prime} e^{\left(\boldsymbol{\tau}_{0} \mathbf{M}_{n}\right)^{\prime}} \epsilon_{n},
\end{aligned}
$$

which are linear and quadratic functions of $\epsilon_{n}$ and have mean zero by verification. Thus we may apply the central limit theorem for linear-quadratic forms in Kelejian and Prucha (2001). The proof of the following proposition is in Appendix C.

Proposition A.2. Under Assumptions 2, 5 and $A .1-A .5, \sqrt{n}\left(\hat{\gamma}_{n}-\gamma_{0}\right) \stackrel{d}{\rightarrow} N\left(0, \lim _{n \rightarrow \infty} \mathbf{C}_{n}^{-1} \boldsymbol{\Omega}_{n} \mathbf{C}_{n}^{-1}\right)$, where $\mathbf{C}_{n}=\frac{1}{n} \mathrm{E} \frac{\partial^{2} Q_{n}\left(\gamma_{0}\right)}{\partial \gamma \partial \gamma^{\prime}}$ is a $3 \times 3$ symmetric block matrix with the $(i, j)$ th element for $1 \leq i, j \leq p$ in the $(1,1)$ th block being

$$
\begin{aligned}
& \frac{2}{n}\left(X_{n} \beta_{0}\right)^{\prime} e^{-\left(\boldsymbol{\alpha}_{0} \mathbf{W}_{n}\right)^{\prime}} \frac{\partial e^{\left(\boldsymbol{\alpha}_{0} \mathbf{W}_{n}\right)^{\prime}}}{\partial \alpha_{i}} e^{\left(\boldsymbol{\tau}_{0} \mathbf{M}_{n}\right)^{\prime}} e^{\boldsymbol{\tau}_{0} \mathbf{M}_{n}} \frac{\partial e^{\boldsymbol{\alpha}_{0} \mathbf{W}_{n}}}{\partial \alpha_{j}} e^{-\boldsymbol{\alpha}_{0} \mathbf{W}_{n}} X_{n} \beta_{0}+\frac{2 \sigma_{0}^{2}}{n} \operatorname{tr}\left(e^{-\left(\boldsymbol{\alpha}_{0} \mathbf{W}_{n}\right)^{\prime}} \frac{\partial^{2} e^{\left(\boldsymbol{\alpha}_{0} \mathbf{W}_{n}\right)^{\prime}}}{\partial \alpha_{i} \partial \alpha_{j}}\right) \\
&+\frac{2 \sigma_{0}^{2}}{n} \operatorname{tr}\left(e^{-\left(\boldsymbol{\tau}_{0} \mathbf{M}_{n}\right)^{\prime}} e^{-\left(\boldsymbol{\alpha}_{0} \mathbf{W}_{n}\right)^{\prime}} \frac{\partial e^{\left(\boldsymbol{\alpha}_{0} \mathbf{W}_{n}\right)^{\prime}}}{\partial \alpha_{i}} e^{\left(\boldsymbol{\tau}_{0} \mathbf{M}_{n}\right)^{\prime}} e^{\boldsymbol{\tau}_{0} \mathbf{M}_{n}} \frac{\partial e^{\boldsymbol{\alpha}_{0} \mathbf{W}_{n}}}{\partial \alpha_{j}} e^{-\boldsymbol{\alpha}_{0} \mathbf{W}_{n}} e^{-\boldsymbol{\tau}_{0} \mathbf{M}_{n}}\right),
\end{aligned}
$$

the $(i, j)$ th element for $1 \leq i \leq p, 1 \leq j \leq q$ in the $(1,2)$ th block being

$$
\frac{2 \sigma_{0}^{2}}{n} \operatorname{tr}\left(e^{-\left(\boldsymbol{\tau}_{0} \mathbf{M}_{n}\right)^{\prime}} e^{-\left(\boldsymbol{\alpha}_{0} \mathbf{W}_{n}\right)^{\prime}} \frac{\partial e^{\left(\boldsymbol{\alpha}_{0} \mathbf{W}_{n}\right)^{\prime}}}{\partial \alpha_{i}} \frac{\partial e^{\left(\boldsymbol{\tau}_{0} \mathbf{M}_{n}\right)^{\prime}}}{\partial \tau_{j}}\right)+\frac{2 \sigma_{0}^{2}}{n} \operatorname{tr}\left(e^{-\left(\boldsymbol{\tau}_{0} \mathbf{M}_{n}\right)^{\prime}} e^{-\left(\boldsymbol{\alpha}_{0} \mathbf{W}_{n}\right)^{\prime}} \frac{\partial e^{\left(\boldsymbol{\alpha}_{0} \mathbf{W}_{n}\right)^{\prime}}}{\partial \alpha_{i}} e^{\left(\boldsymbol{\tau}_{0} \mathbf{M}_{n}\right)^{\prime}} \frac{\partial e^{\boldsymbol{\tau}_{0} \mathbf{M}_{n}}}{\partial \tau_{j}} e^{-\boldsymbol{\tau}_{0} \mathbf{M}_{n}}\right),
$$


the ith row for $1 \leq i \leq p$ in the $(1,3)$ th block being $-2\left(X_{n} \beta_{0}\right)^{\prime} e^{-\left(\boldsymbol{\alpha}_{0} \mathbf{W}_{n}\right)^{\prime}} \frac{\partial e^{\left(\boldsymbol{\alpha}_{0} \mathbf{W}_{n}\right)^{\prime}}}{\partial \alpha_{i}} e^{\left(\boldsymbol{\tau}_{0} \mathbf{M}_{n}\right)^{\prime}} e^{\boldsymbol{\tau}_{0} \mathbf{M}_{n}} X_{n}$, the $(i, j)$ th element for $1 \leq i, j \leq q$ in the $(2,2)$ th block being

$$
\frac{2 \sigma_{0}^{2}}{n} \operatorname{tr}\left(e^{-\left(\boldsymbol{\tau}_{0} \mathbf{M}_{n}\right)^{\prime}} \frac{\partial^{2} e^{\left(\boldsymbol{\tau}_{0} \mathbf{M}_{n}\right)^{\prime}}}{\partial \tau_{i} \partial \tau_{j}}\right)+\frac{2 \sigma_{0}^{2}}{n} \operatorname{tr}\left(e^{-\left(\boldsymbol{\tau}_{0} \mathbf{M}_{n}\right)^{\prime}} \frac{\partial e^{\left(\boldsymbol{\tau}_{0} \mathbf{M}_{n}\right)^{\prime}}}{\partial \tau_{i}} \frac{\partial e^{\boldsymbol{\tau}_{0} \mathbf{M}_{n}}}{\partial \tau_{j}} e^{-\boldsymbol{\tau}_{0} \mathbf{M}_{n}}\right)
$$

the elements in the $(2,3)$ th block being all zero, and the $(3,3)$ th block being $\frac{2}{n} X_{n}^{\prime} e^{\left(\boldsymbol{\tau}_{0} \mathbf{M}_{n}\right)^{\prime}} e^{\boldsymbol{\tau}_{0} \mathbf{M}_{n}} X_{n}$, and $\boldsymbol{\Omega}_{n}=\mathbf{C}_{n}+\boldsymbol{\Omega}_{1 n}$, where $\boldsymbol{\Omega}_{1 n}$ is a symmetric $3 \times 3$ block matrix with the $(i, j)$ th element for $1 \leq i, j \leq p$ in the $(1,1)$ th block being

$$
\begin{aligned}
& \left(\mu_{4}-3 \sigma_{0}^{4}\right) \operatorname{vec}_{\mathrm{D}}{ }^{\prime}\left(2 e^{-\left(\boldsymbol{\tau}_{0} \mathbf{M}_{n}\right)^{\prime}} e^{-\left(\boldsymbol{\alpha}_{0} \mathbf{W}_{n}\right)^{\prime}} \frac{\partial e^{\left(\boldsymbol{\alpha}_{0} \mathbf{W}_{n}\right)^{\prime}}}{\partial \alpha_{i}} e^{\left(\boldsymbol{\tau}_{0} \mathbf{M}_{n}\right)^{\prime}}\right) \operatorname{vec}_{\mathrm{D}}\left(2 e^{-\left(\boldsymbol{\tau}_{0} \mathbf{M}_{n}\right)^{\prime}} e^{-\left(\boldsymbol{\alpha}_{0} \mathbf{W}_{n}\right)^{\prime}} \frac{\partial e^{\left(\boldsymbol{\alpha}_{0} \mathbf{W}_{n}\right)^{\prime}}}{\partial \alpha_{j}} e^{\left(\boldsymbol{\tau}_{0} \mathbf{M}_{n}\right)^{\prime}}\right) \\
& +2 \mu_{3}\left(X_{n} \beta_{0}\right)^{\prime} e^{-\left(\boldsymbol{\alpha}_{0} \mathbf{W}_{n}\right)^{\prime}} \frac{\partial e^{\left(\boldsymbol{\alpha}_{0} \mathbf{W}_{n}\right)^{\prime}}}{\partial \alpha_{i}} e^{\left(\boldsymbol{\tau}_{0} \mathbf{M}_{n}\right)^{\prime}} \operatorname{vec}_{\mathrm{D}}\left(2 e^{-\left(\boldsymbol{\tau}_{0} \mathbf{M}_{n}\right)^{\prime}} e^{-\left(\boldsymbol{\alpha}_{0} \mathbf{W}_{n}\right)^{\prime}} \frac{\partial e^{\left(\boldsymbol{\alpha}_{0} \mathbf{W}_{n}\right)^{\prime}}}{\partial \alpha_{j}} e^{\left(\boldsymbol{\tau}_{0} \mathbf{M}_{n}\right)^{\prime}}\right) \\
& +2 \mu_{3}\left(X_{n} \beta_{0}\right)^{\prime} e^{-\left(\boldsymbol{\alpha}_{0} \mathbf{W}_{n}\right)^{\prime}} \frac{\partial e^{\left(\boldsymbol{\alpha}_{0} \mathbf{W}_{n}\right)^{\prime}}}{\partial \alpha_{j}} e^{\left(\boldsymbol{\tau}_{0} \mathbf{M}_{n}\right)^{\prime}} \operatorname{vec}_{\mathrm{D}}\left(2 e^{-\left(\boldsymbol{\tau}_{0} \mathbf{M}_{n}\right)^{\prime}} e^{-\left(\boldsymbol{\alpha}_{0} \mathbf{W}_{n}\right)^{\prime}} \frac{\partial e^{\left(\boldsymbol{\alpha}_{0} \mathbf{W}_{n}\right)^{\prime}}}{\partial \alpha_{i}} e^{\left(\boldsymbol{\tau}_{0} \mathbf{M}_{n}\right)^{\prime}}\right)
\end{aligned}
$$

the $(i, j)$ th element for $1 \leq i \leq p, 1 \leq j \leq q$ in the $(1,2)$ th block being

$$
\begin{aligned}
& \left(\mu_{4}-3 \sigma_{0}^{4}\right) \operatorname{vec}_{\mathrm{D}}{ }^{\prime}\left(2 e^{-\left(\boldsymbol{\tau}_{0} \mathbf{M}_{n}\right)^{\prime}} e^{-\left(\boldsymbol{\alpha}_{0} \mathbf{W}_{n}\right)^{\prime}} \frac{\partial e^{\left(\boldsymbol{\alpha}_{0} \mathbf{W}_{n}\right)^{\prime}}}{\partial \alpha_{i}} e^{\left(\boldsymbol{\tau}_{0} \mathbf{M}_{n}\right)^{\prime}}\right) \operatorname{vec}_{\mathrm{D}}\left(2 e^{-\left(\boldsymbol{\tau}_{0} \mathbf{M}_{n}\right)^{\prime}} \frac{\partial e^{\left(\boldsymbol{\tau}_{0} \mathbf{M}_{n}\right)^{\prime}}}{\partial \tau_{j}}\right) \\
& +2 \mu_{3}\left(X_{n} \beta_{0}\right)^{\prime} e^{-\left(\boldsymbol{\alpha}_{0} \mathbf{W}_{n}\right)^{\prime}} \frac{\partial e^{\left(\boldsymbol{\alpha}_{0} \mathbf{W}_{n}\right)^{\prime}}}{\partial \alpha_{i}} e^{\left(\boldsymbol{\tau}_{0} \mathbf{M}_{n}\right)^{\prime}} \operatorname{vec}_{\mathrm{D}}\left(2 e^{-\left(\boldsymbol{\tau}_{0} \mathbf{M}_{n}\right)^{\prime}} \frac{\partial e^{\left(\boldsymbol{\tau}_{0} \mathbf{M}_{n}\right)^{\prime}}}{\partial \tau_{j}}\right)
\end{aligned}
$$

the ith row for $1 \leq i \leq p$ in the $(1,3)$ th block being $-2 \mu_{3} \operatorname{vec}_{\mathrm{D}}{ }^{\prime}\left(2 e^{-\left(\boldsymbol{\tau}_{0} \mathbf{M}_{n}\right)^{\prime}} e^{-\left(\boldsymbol{\alpha}_{0} \mathbf{W}_{n}\right)^{\prime}} \frac{\partial e^{\left(\boldsymbol{\alpha}_{0} \mathbf{W}_{n}\right)^{\prime}}}{\partial \alpha_{i}} e^{\left(\boldsymbol{\tau}_{0} \mathbf{M}_{n}\right)^{\prime}}\right) e^{\boldsymbol{\tau}_{0} \mathbf{M}_{n}} X_{n}$, the $(i, j)$ th element for $1 \leq i, j \leq q$ in the $(2,2)$ th block being

$$
\left(\mu_{4}-3 \sigma_{0}^{4}\right) \operatorname{vec}_{\mathrm{D}}^{\prime}\left(2 e^{-\left(\boldsymbol{\tau}_{0} \mathbf{M}_{n}\right)^{\prime}} \frac{\partial e^{\left(\boldsymbol{\tau}_{0} \mathbf{M}_{n}\right)^{\prime}}}{\partial \tau_{i}}\right) \operatorname{vec}_{\mathrm{D}}\left(2 e^{-\left(\boldsymbol{\tau}_{0} \mathbf{M}_{n}\right)^{\prime}} \frac{\partial e^{\left(\boldsymbol{\tau}_{0} \mathbf{M}_{n}\right)^{\prime}}}{\partial \tau_{j}}\right)
$$

the ith row for $1 \leq i \leq q$ in the $(2,3)$ th block being $-2 \mu_{3} \operatorname{vec}_{\mathrm{D}}{ }^{\prime}\left(2 e^{-\left(\boldsymbol{\tau}_{0} \mathbf{M}_{n}\right)^{\prime}} \frac{\partial e^{\left(\boldsymbol{\tau}_{0} \mathbf{M}_{n}\right)^{\prime}}}{\partial \tau_{i}}\right) e^{\boldsymbol{\tau}_{0} \mathbf{M}_{n}} X_{n}$, and the elements of the $(3,3)$ th blocks being zero.

When $\mu_{3}=\mu_{4}-3 \sigma_{0}^{4}=0, \Omega_{1 n}=0$.

\section{Appendix B. Lemmas}

Lemmas 2-5 can be found in, e.g., Lin and Lee (2010) and Jin and Lee (2012). The central limit theorem in Lemma 5 is originated in Kelejian and Prucha (2010). Lemma 6 is Lemma A.6 in Lee (2007). They are provided here for easy reference. Let UB stand for "bounded in both row and column sum norms". 
Lemma 2. Suppose that $n \times n$ matrices $\left\{A_{n}\right\}$ are UB. Elements of $n \times k$ matrices $\left\{X_{n}\right\}$ are uniformly bounded and $\lim _{n \rightarrow \infty} n^{-1} X_{n}^{\prime} X_{n}$ exists and is nonsingular. Let $M_{n}=I_{n}-X_{n}\left(X_{n}^{\prime} X_{n}\right)^{-1} X_{n}^{\prime}$. Then $\left\{M_{n}\right\}$ are $U B$ and $\operatorname{tr}\left(M_{n} A_{n}\right)=\operatorname{tr}\left(A_{n}\right)+O(1)$.

Lemma 3. Suppose that $A_{n}=\left[a_{n, i j}\right]$ and $B_{n}=\left[b_{n, i j}\right]$ are two $n \times n$ matrices and $\epsilon_{n i}$ 's in $\epsilon_{n}=\left(\epsilon_{n 1}, \ldots, \epsilon_{n n}\right)^{\prime}$ are independently distributed with mean zero (but may not be i.i.d.). Then,

(1) $\mathrm{E}\left(\epsilon_{n} \cdot \epsilon_{n}^{\prime} A_{n} \epsilon_{n}\right)=\left(a_{n, 11} \mathrm{E}\left(\epsilon_{n 1}^{3}\right), \ldots, a_{n, n n} \mathrm{E}\left(\epsilon_{n n}^{3}\right)\right)^{\prime}$, and

(2) $\mathrm{E}\left(\epsilon_{n}^{\prime} A_{n} \epsilon_{n} \cdot \epsilon_{n}^{\prime} B_{n} \epsilon_{n}\right)=\sum_{i=1}^{n} a_{n, i i} b_{n, i i}\left[\mathrm{E}\left(\epsilon_{n i}^{4}\right)-3 \sigma_{n i}^{4}\right]+\operatorname{tr}\left(\Sigma_{n} A_{n}\right) \operatorname{tr}\left(\Sigma_{n} B_{n}\right)+\operatorname{tr}\left[\Sigma_{n} A_{n} \Sigma_{n}\left(B_{n}+B_{n}^{\prime}\right)\right]$, where $\Sigma_{n}=\operatorname{Diag}\left(\sigma_{n 1}^{2}, \ldots, \sigma_{n n}^{2}\right)$ with $\sigma_{n i}^{2}=\mathrm{E}\left(\epsilon_{n i}^{2}\right), i=1, \ldots, n$.

Lemma 4. Suppose that $n \times n$ matrices $\left\{A_{n}\right\}$ are UB, elements of the $n \times k$ matrices $\left\{C_{n}\right\}$ are uniformly bounded, and $\epsilon_{n i}$ 's in $\epsilon_{n}=\left(\epsilon_{n 1}, \ldots, \epsilon_{n n}\right)^{\prime}$ are independent random variables with mean zero and variance $\sigma_{n i}^{2}$. The sequence $\left\{\mathrm{E}\left(\epsilon_{n i}^{4}\right)\right\}$ is bounded. Then $\epsilon_{n}^{\prime} A_{n} \epsilon_{n}=O_{P}(n), \mathrm{E}\left(\epsilon_{n}^{\prime} A_{n} \epsilon_{n}\right)=O(n), n^{-1}\left[\epsilon_{n}^{\prime} A_{n} \epsilon_{n}-\mathrm{E}\left(\epsilon_{n}^{\prime} A_{n} \epsilon_{n}\right)\right]=$ $o_{P}(1)$ and $n^{-1 / 2} C_{n}^{\prime} A_{n} \epsilon_{n}=O_{P}(1)$.

Lemma 5. Suppose that $\left\{A_{n}\right\}$ is a sequence of symmetric $n \times n$ matrices that are $U B$ and $b_{n}=\left(b_{n 1}, \ldots, b_{n n}\right)^{\prime}$ is an $n$-dimensional column vector such that $\sup _{n} n^{-1} \sum_{i=1}^{n}\left|b_{n i}\right|^{2+\eta_{1}}<\infty$ for some $\eta_{1}>0$. Furthermore, suppose that $\epsilon_{n 1}, \cdots, \epsilon_{n n}$ are mutually independent with zero means and the moments $\mathrm{E}\left(\left|\epsilon_{n i}\right|^{4+\eta_{2}}\right)$ for some $\eta_{2}>0$ exist and are uniformly bounded for all $n$ and $i$. Let $\sigma_{c_{n}}^{2}$ be the variance of $c_{n}$ where $c_{n}=\epsilon_{n}^{\prime} A_{n} \epsilon_{n}+b_{n}^{\prime} \epsilon_{n}-\operatorname{tr}\left(A_{n} \Sigma_{n}\right)$ with $\Sigma_{n}$ being a diagonal matrix with $\mathrm{E} \epsilon_{n i}^{2}$ 's on its diagonal. Assume that $n^{-1} \sigma_{c_{n}}^{2}$ is bounded away from zero. Then $\frac{c_{n}}{\sigma_{c_{n}}} \stackrel{d}{\rightarrow} N(0,1)$.

Lemma 6. Suppose that $\left[Q_{n}(\gamma)-\bar{Q}_{n}(\gamma)\right]$ converges in probability to zero uniformly in $\gamma \in \Gamma$ which is a convex set, and $\left\{\bar{Q}_{n}(\gamma)\right\}$ satisfies the identification uniqueness condition at $\gamma_{0}$. Let $\hat{\gamma}_{n}$ and $\hat{\gamma}_{n}^{*}$ be, respectively, the minimizers of $Q_{n}(\gamma)$ and $Q_{n}^{*}(\gamma)$ in $\Gamma$. If $Q_{n}^{*}(\gamma)-Q_{n}(\gamma)=o_{P}(1)$ uniformly in $\gamma \in \Gamma$, then both $\hat{\gamma}_{n}$ and $\hat{\gamma}_{n}^{*}$ converge in probability to $\gamma_{0}$.

In addition, suppose that $\frac{\partial^{2} Q_{n}(\gamma)}{\partial \gamma \partial \gamma^{\prime}}$ converges in probability to a well defined limiting matrix, uniformly in $\gamma \in \Gamma$, which is nonsingular at $\gamma_{0}$, and $\sqrt{n} \frac{\partial Q_{n}\left(\gamma_{0}\right)}{\partial \gamma}=O_{P}(1)$. If $\frac{\partial^{2} Q_{n}^{*}(\gamma)}{\partial \gamma \partial \gamma^{\prime}}-\frac{\partial^{2} Q_{n}(\gamma)}{\partial \gamma \partial \gamma^{\prime}}=o_{P}(1)$ uniformly in $\gamma \in \Gamma$ and $\sqrt{n} \frac{\partial Q_{n}^{*}\left(\gamma_{0}\right)}{\partial \gamma}-\sqrt{n} \frac{\partial Q_{n}\left(\gamma_{0}\right)}{\partial \gamma}=o_{P}(1)$, then $\sqrt{n}\left(\hat{\gamma}_{n}^{*}-\gamma_{0}\right)$ and $\sqrt{n}\left(\hat{\gamma}_{n}-\gamma_{0}\right)$ have the same limiting distribution.

For the best GMME in the homoskedastic case, we show that adding any other moments to the selected ones cannot improve the asymptotic efficiency using the redundancy conditions in Breusch et al. (1999). Suppose that we have a set of moment conditions $\mathrm{E}\left[g_{n}^{*}(\gamma)\right]=0$ with the corresponding optimal GMME being $\hat{\gamma}_{n}^{*}$. Adding some additional moment conditions $\mathrm{E}\left[g_{n}(\gamma)\right]=0$ to $\mathrm{E}\left[g_{n}^{*}(\gamma)\right]=0$, we have an optimal GMME $\hat{\gamma}_{n}$ using both sets of moment conditions. Then the moment conditions $\mathrm{E}\left[g_{n}(\gamma)\right]=0$ are redundant given $\mathrm{E}\left[g_{n}^{*}(\gamma)\right]=0$ if the asymptotic variances of $\hat{\gamma}_{n}^{*}$ and $\hat{\gamma}_{n}$ are the same. Let $V_{n}^{*}=n \mathrm{E}\left[g_{n}^{*}\left(\gamma_{0}\right) g_{n}^{*^{\prime}}\left(\gamma_{0}\right)\right]$, 
$V_{n, 21}=n \mathrm{E}\left[g_{n}\left(\gamma_{0}\right) g_{n}^{*^{\prime}}\left(\gamma_{0}\right)\right], G_{n}^{*}=\mathrm{E} \frac{\partial g_{n}^{*}\left(\gamma_{0}\right)}{\partial \gamma^{\prime}}$ and $G_{n}=\mathrm{E} \frac{\partial g_{n}\left(\gamma_{0}\right)}{\partial \gamma^{\prime}}$. The following two lemmas from Breusch et al. (1999) give conditions for moment redundancy.

Lemma 7. The following statements are equivalent: (a) $\mathrm{E}\left[g_{n}(\gamma)\right]=0$ is redundant given $\mathrm{E}\left[g_{n}^{*}(\gamma)\right]=0 ;(b)$ $G_{n}=V_{n, 21} V_{n}^{*-1} G_{n}^{*}$; and (c) there exists a matrix $T$ such that $G_{n}^{*}=V_{n}^{*} T$ and $G_{n}=V_{n, 21} T$.

Lemma 8. Let the set of moment conditions to be considered be $\mathrm{E}\left[g_{n}(\gamma)\right]=\mathrm{E}\left[g_{1 n}^{\prime}(\gamma), g_{2 n}^{\prime}(\gamma), g_{3 n}^{\prime}(\gamma)\right]^{\prime}=0$, or simply $g=\left(g_{1}^{\prime}, g_{2}^{\prime}, g_{3}^{\prime}\right)^{\prime}$. Then $\left(g_{2}^{\prime}, g_{3}^{\prime}\right)^{\prime}$ is redundant given $g_{1}$ if and only if $g_{2}$ is redundant given $g_{1}$ and $g_{3}$ is redundant given $g_{1}$.

The following Lemmas 9, 10 and 11 summarize relevant matrices of the MESS which possess the essential UB property. ${ }^{34}$

Lemma 9. Suppose that $n \times n$ matrices $\left\{M_{n 1}\right\}, \ldots,\left\{M_{n q}\right\}$ are UB. The smallest eigenvalue of $e^{\left(\boldsymbol{\tau} \mathbf{M}_{n}\right)^{\prime}} e^{\tau \mathbf{M}_{n}}$ is bounded away from zero uniformly over the interval $[-\delta, \delta]^{q}$ for some finite $\delta>0$. Elements of the $n \times k$ matrix $X_{n}$ are uniformly bounded. The limit of $\frac{1}{n} X_{n}^{\prime} e^{\left(\boldsymbol{\tau} \mathbf{M}_{n}\right)^{\prime}} e^{\tau \mathbf{M}_{n}} X_{n}$ exists and is nonsingular for any $\boldsymbol{\tau} \in$ $[-\delta, \delta]^{q}$. Then $e^{\tau \mathbf{M}_{n}}, X_{n}\left(X_{n}^{\prime} e^{\left(\tau \mathbf{M}_{n}\right)^{\prime}} e^{\tau \mathbf{M}_{n}} X_{n}\right)^{-1} X_{n}^{\prime}$ and $H_{n}(\boldsymbol{\tau})=I_{n}-e^{\tau \mathbf{M}_{n}} X_{n}\left(X_{n}^{\prime} e^{\left(\boldsymbol{\tau} \mathbf{M}_{n}\right)^{\prime}} e^{\tau \mathbf{M}_{n}} X_{n}\right)^{-1} X_{n}^{\prime} e^{\left(\tau \mathbf{M}_{n}\right)^{\prime}}$ are UB uniformly in $\boldsymbol{\tau} \in[-\delta, \delta]^{q}$.

Lemma 10. Let $W_{n 1}, \ldots, W_{n p}, M_{n 1}, \ldots, M_{n q}, A_{n}$ and $B_{n}$ be $n \times n$ matrices that are $U B, b_{n}$ be an $n$-dimensional vector with uniformly bounded elements, $X_{n}$ be an $n \times k$ matrix with uniformly bounded elements, and $\epsilon_{n}=\left(\epsilon_{n 1}, \ldots, \epsilon_{n n}\right)^{\prime}$ be a random vector with independent elements that have mean zero and variances $\sigma_{n i}^{2}$ 's. Assume that $\lim _{n \rightarrow \infty} \frac{1}{n} X_{n}^{\prime} e^{\left(\boldsymbol{\tau} \mathbf{M}_{n}\right)^{\prime}} e^{\tau \mathbf{M}_{n}} X_{n}$ exists and is nonsingular for any $\boldsymbol{\tau} \in[-\delta, \delta]^{q}$ and the sequence $\left\{\mathrm{E}\left(\epsilon_{n i}^{4}\right)\right\}$ is bounded. Then $\frac{1}{n} b_{n}^{\prime} e^{\left(\boldsymbol{\alpha} \mathbf{W}_{n}\right)^{\prime}} e^{\left(\boldsymbol{\tau} \mathbf{M}_{n}\right)^{\prime}} H_{n}(\boldsymbol{\tau}) e^{\boldsymbol{\tau} \mathbf{M}_{n}} e^{\boldsymbol{\alpha} \mathbf{W}_{n}} A_{n} \epsilon_{n}=o_{P}(1)$ uniformly on the parameter space $\boldsymbol{\Phi}=[-\delta, \delta]^{p+q}, \frac{1}{n} b_{n}^{\prime} e^{\left(\boldsymbol{\alpha} \mathbf{W}_{n}\right)^{\prime}} e^{\left(\boldsymbol{\tau} \mathbf{M}_{n}\right)^{\prime}} B_{n} e^{\boldsymbol{\tau} \mathbf{M}_{n}} e^{\boldsymbol{\alpha} \mathbf{W}_{n}} A_{n} \epsilon_{n}=o_{P}(1)$ uniformly on $\boldsymbol{\Phi}, \frac{1}{n}\left[\epsilon_{n}^{\prime} A_{n}^{\prime} e^{\left(\boldsymbol{\alpha} \mathbf{W}_{n}\right)^{\prime}} e^{\left(\boldsymbol{\tau} \mathbf{M}_{n}\right)^{\prime}} H_{n}(\boldsymbol{\tau}) e^{\boldsymbol{\tau} \mathbf{M}_{n}} e^{\boldsymbol{\alpha} \mathbf{W}_{n}} A_{n} \epsilon_{n}-\operatorname{tr}\left(A_{n}^{\prime} e^{\left(\boldsymbol{\alpha} \mathbf{W}_{n}\right)^{\prime}} e^{\left(\boldsymbol{\tau} \mathbf{M}_{n}\right)^{\prime}} H_{n}(\boldsymbol{\tau}) e^{\boldsymbol{\tau} \mathbf{M}_{n}} e^{\boldsymbol{\alpha} \mathbf{W}_{n}} A_{n} \Sigma_{n}\right)\right]=o_{P}(1)$ uniformly on $\boldsymbol{\Phi}, \frac{1}{n}\left[\epsilon_{n}^{\prime} A_{n}^{\prime} e^{\left(\boldsymbol{\alpha} \mathbf{W}_{n}\right)^{\prime}} e^{\left(\boldsymbol{\tau} \mathbf{M}_{n}\right)^{\prime}} B_{n} e^{\tau \mathbf{M}_{n}} e^{\boldsymbol{\alpha} \mathbf{W}_{n}} A_{n} \epsilon_{n}-\operatorname{tr}\left(A_{n}^{\prime} e^{\left(\boldsymbol{\alpha} \mathbf{W}_{n}\right)^{\prime}} e^{\left(\boldsymbol{\tau} \mathbf{M}_{n}\right)^{\prime}} B_{n} e^{\tau \mathbf{M}_{n}} e^{\boldsymbol{\alpha} \mathbf{W}_{n}} A_{n} \Sigma_{n}\right)\right]=$ $o_{P}(1)$ uniformly on $\boldsymbol{\Phi}$, and $\frac{1}{n} \operatorname{tr}\left(A_{n}^{\prime} e^{\left(\boldsymbol{\alpha} \mathbf{W}_{n}\right)^{\prime}} e^{\left(\boldsymbol{\tau} \mathbf{M}_{n}\right)^{\prime}}\left(I_{n}-H_{n}(\boldsymbol{\tau})\right) e^{\boldsymbol{\tau} \mathbf{M}_{n}} e^{\boldsymbol{\alpha} \mathbf{W}_{n}} A_{n} \Sigma_{n}\right)=o(1)$ uniformly on $\boldsymbol{\Phi}$, where $H_{n}(\boldsymbol{\tau})=I_{n}-e^{\boldsymbol{\tau} \mathbf{M}_{n}} X_{n}\left(X_{n}^{\prime} e^{\left(\boldsymbol{\tau} \mathbf{M}_{n}\right)^{\prime}} e^{\boldsymbol{\tau} \mathbf{M}_{n}} X_{n}\right)^{-1} X_{n}^{\prime} e^{\left(\boldsymbol{\tau} \mathbf{M}_{n}\right)^{\prime}}$ and $\Sigma_{n}=\operatorname{Diag}\left(\sigma_{n 1}^{2}, \ldots, \sigma_{n n}^{2}\right)$.

Lemma 11. Let $A_{n}$ be any $n \times n$ UB matrix and $a_{n}=o_{P}(1)$. Then $\left\|e^{a_{n} A_{n}}-I_{n}\right\|_{\infty}=o_{P}(1)$ and $\| e^{a_{n} A_{n}}-$ $I_{n} \|_{1}=o_{P}(1)$.

\section{Appendix C. Proofs}

Proof of Proposition 8. To show that $\hat{\gamma}_{n}^{*}$ is the best GMME within the class of GMMEs with linear and quadratic moments, we prove that the moment condition $\mathrm{E}\left[g_{n}(\gamma)\right]=0$, where $g_{n}(\gamma)$ is a set of arbitrary linear

\footnotetext{
${ }^{34}$ The detailed arguments to establish their UB properties are in a supplementary file available upon request.
} 
and quadratic moments in (25), is redundant given the moment conditions $\mathrm{E}\left[g_{n}^{*}(\gamma)\right]=0$. By Lemmas 7 and 8, it is sufficient to show that there exists a matrix $T$ such that $G_{n}=\mathrm{E} \frac{\partial g_{n}\left(\gamma_{0}\right)}{\partial \gamma^{\prime}}=V_{n, 21} T$ and $G_{n}^{*}=V_{n}^{*} T$, where

$$
\begin{aligned}
V_{n, 21}= & n \mathrm{E}\left(g_{n}\left(\gamma_{0}\right) g_{n}^{*^{\prime}}\left(\gamma_{0}\right)\right) \\
= & \frac{1}{n}\left(\begin{array}{cccc}
\sigma_{0}^{4} \operatorname{tr}\left(P_{n 1}^{s} P_{n 1}^{*}\right) & \ldots & \sigma_{0}^{4} \operatorname{tr}\left(P_{n 1}^{s} P_{n, k^{*}+4}^{*}\right) & \mu_{3} \operatorname{vec}_{\mathrm{D}}^{\prime}\left(P_{n 1}\right) F_{n}^{*} \\
\vdots & \ddots & \vdots & \vdots \\
\sigma_{0}^{4} \operatorname{tr}\left(P_{n, k_{p}}^{s} P_{n 1}^{*}\right) & \ldots & \sigma_{0}^{4} \operatorname{tr}\left(P_{n, k_{p}}^{s} P_{n, k^{*}+4}^{*}\right) & \mu_{3} \operatorname{vec}_{\mathrm{D}}{ }^{\prime}\left(P_{n, k_{p}}\right) F_{n}^{*} \\
\mu_{3} F_{n}^{\prime} \operatorname{vec}_{\mathrm{D}}\left(P_{n 1}^{*}\right) & \ldots & \mu_{3} F_{n}^{\prime} \operatorname{vec}_{\mathrm{D}}\left(P_{n, k^{*}+4}^{*}\right) & \sigma_{0}^{2} F_{n}^{\prime} F_{n}^{*}
\end{array}\right) \\
& +\frac{1}{n}\left(\mu_{4}-3 \sigma_{0}^{4}\right)\left(\begin{array}{cccc}
\operatorname{vec}_{\mathrm{D}}{ }^{\prime}\left(P_{n 1}\right) \operatorname{vec}_{\mathrm{D}}\left(P_{n 1}^{*}\right) & \ldots & \operatorname{vec}_{\mathrm{D}}^{\prime}\left(P_{n 1}\right) \operatorname{vec}_{\mathrm{D}}\left(P_{n, k^{*}+4}^{*}\right) & 0 \\
\vdots & \ddots & \vdots & \vdots \\
\operatorname{vec}_{\mathrm{D}}{ }^{\prime}\left(P_{n, k_{p}}\right) \operatorname{vec}_{\mathrm{D}}\left(P_{n 1}^{*}\right) & \ldots & \operatorname{vec}_{\mathrm{D}}{ }^{\prime}\left(P_{n, k_{p}}\right) \operatorname{vec}_{\mathrm{D}}\left(P_{n, k^{*}+4}^{*}\right) & 0 \\
0 & \ldots & 0 & 0
\end{array}\right)
\end{aligned}
$$

by Lemma 3.

Let $P_{\alpha n}^{*}=P_{n 1}^{*}-\frac{\left(\eta_{4}-3\right)-\eta_{3}^{2}}{\left(\eta_{4}-1\right)-\eta_{3}^{2}} P_{n 2}^{*}-\frac{\sigma_{0}^{-1} \eta_{3}}{\left(\eta_{4}-1\right)-\eta_{3}^{2}} P_{n 3}^{*}, P_{\tau n}^{*}=M_{n}, P_{\beta n l}^{*}=P_{n, l+4}^{*}$ for $l=1, \ldots, k^{*}$, and $F_{\alpha n}^{*}=$ $\frac{\eta_{4}-1}{\left(\eta_{4}-1\right)-\eta_{3}^{2}} F_{n 2}^{*}-\frac{\eta_{3}^{2}}{\left(\eta_{4}-1\right)-\eta_{3}^{2}} F_{n 3}^{*}\left(\frac{1}{n} l_{n}^{\prime} e^{\tau_{0} M_{n}} W_{n} X_{n} \beta_{0}\right)-\frac{2 \sigma_{0} \eta_{3}}{\left(\eta_{4}-1\right)-\eta_{3}^{2}} F_{n 4}^{*}$. If $e^{\tau_{0} M_{n}} X_{n}$ does not contain an intercept term, let $F_{\beta n}^{*}=\frac{\eta_{4}-1}{\left(\eta_{4}-1\right)-\eta_{3}^{2}} F_{n 1}^{*}-\frac{\eta_{3}^{2}}{\left(\eta_{4}-1\right)-\eta_{3}^{2}} F_{n 3}^{*}\left(\frac{1}{n} l_{n}^{\prime} e^{\tau_{0} M_{n}} X_{n}^{*}\right)$; otherwise, let $F_{\beta n}^{*}=\frac{\eta_{4}-1}{\left(\eta_{4}-1\right)-\eta_{3}^{2}} F_{n 1}^{*}\left(I_{k^{*}}, 0_{k^{*} \times 1}\right)+$ $\frac{\eta_{4}-1}{\left(\eta_{4}-1\right)-\eta_{3}^{2}} c\left(\tau_{0}\right) F_{n 3}^{*} e_{k k}^{\prime}-\frac{\eta_{3}^{2}}{\left(\eta_{4}-1\right)-\eta_{3}^{2}} F_{n 3}^{*}\left(\frac{1}{n} l_{n}^{\prime} e^{\tau_{0} M_{n}} X_{n}\right)$, where $e_{k j}$ is the $j$ th unit vector in $R^{k}$. Then

$$
\epsilon_{n}^{\prime}(\gamma)\left(P_{\alpha n}^{*} \epsilon_{n}(\gamma), P_{\tau n}^{*} \epsilon_{n}(\gamma), P_{\beta n 1}^{*} \epsilon_{n}(\gamma), \ldots, P_{\beta n k^{*}}^{*} \epsilon_{n}(\gamma)\right)=\epsilon_{n}^{\prime}(\gamma)\left(P_{n 1}^{*} \epsilon_{n}(\gamma), \ldots, P_{n, k^{*}+4}^{*} \epsilon_{n}(\gamma)\right) \Delta_{P}
$$

where

$$
\Delta_{P}^{\prime}=\left(\begin{array}{ccccc}
1 & -\frac{\left(\eta_{4}-3\right)-\eta_{3}^{2}}{\left(\eta_{4}-1\right)-\eta_{3}^{2}} & -\frac{\sigma_{0}^{-1} \eta_{3}}{\left(\eta_{4}-1\right)-\eta_{3}^{2}} & 0 & 0 \\
0 & 0 & 0 & 1 & 0 \\
0 & 0 & 0 & 0 & I_{k^{*}}
\end{array}\right) .
$$

If $e^{\eta_{0} M_{n}} X_{n}$ does not contain an intercept term, $\left(F_{\alpha n}^{*}, F_{\beta n}^{*}\right)=\left(F_{n 1}^{*}, F_{n 2}^{*}, F_{n 3}^{*}, F_{n 4}^{*}\right) \Delta_{F 1}$, where

$$
\Delta_{F 1}^{\prime}=\left(\begin{array}{cccc}
0 & \frac{\eta_{4}-1}{\left(\eta_{4}-1\right)-\eta_{3}^{2}} & -\frac{\eta_{3}^{2}}{\left(\eta_{4}-1\right)-\eta_{3}^{2}}\left(\frac{1}{n} l_{n}^{\prime} e^{\tau_{0} M_{n}} W_{n} X_{n} \beta_{0}\right) & -\frac{2 \sigma_{0} \eta_{3}}{\left(\eta_{4}-1\right)-\eta_{3}^{2}} \\
\frac{\eta_{4}-1}{\eta_{4}-1-\eta_{3}^{2}} I_{k^{*}} & 0 & -\frac{\eta_{3}^{2}}{\left(\eta_{4}-1\right)-\eta_{3}^{2}}\left(\frac{1}{n} l_{n}^{\prime} e^{\tau_{0} M_{n}} X_{n}^{*}\right)^{\prime} & 0
\end{array}\right)
$$


otherwise, $\left(F_{\alpha n}^{*}, F_{\beta n}^{*}\right)=\left(F_{n 1}^{*}, F_{n 2}^{*}, F_{n 3}^{*}, F_{n 4}^{*}\right) \Delta_{F 2}$, where

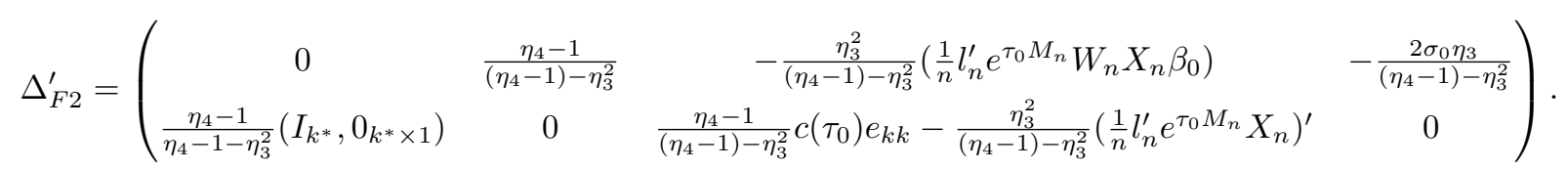

Let $\Delta_{P F}=\left(\begin{array}{cc}\Delta_{P} & 0 \\ 0 & \Delta_{F 1}\end{array}\right)$ if $e^{\eta_{0} M_{n}} X_{n}$ does not contain an intercept term and $\Delta_{P F}=\left(\begin{array}{cc}\Delta_{P} & 0 \\ 0 & \Delta_{F 2}\end{array}\right)$ otherwise. Then $g_{n}^{*^{\prime}}(\gamma) \Delta_{P F}=\epsilon_{n}^{\prime}(\gamma)\left(P_{\alpha n}^{*} \epsilon_{n}(\gamma), P_{\tau n}^{*} \epsilon_{n}(\gamma), P_{\beta n 1}^{*} \epsilon_{n}(\gamma), \ldots, P_{\beta n k^{*}}^{*} \epsilon_{n}(\gamma),\left(F_{\alpha n}^{*}, F_{\beta n}^{*}\right)\right)$. Let

$$
\Delta_{T}^{\prime}=\left(\begin{array}{cccc}
\sigma_{0}^{-2} & 0 & 0 & \left(\sigma_{0}^{-2}, 0\right) \\
0 & \sigma_{0}^{-2} & 0 & (0,0) \\
0 & 0 & b^{\prime} & \left(0,-\sigma_{0}^{-2} I_{k}\right)
\end{array}\right),
$$

where $b^{\prime}=\left(b_{1}^{\prime}, \ldots, b_{k^{*}}^{\prime}\right)$ with $b_{l}=\frac{\sigma_{0}^{-3} \eta_{3}}{\left(\eta_{4}-1\right)-\eta_{3}^{2}} e_{k l}^{\prime}$. Define $T=\Delta_{P F} \Delta_{T}$. We shall show that $G_{n}=V_{n, 21} T$ and $G_{n}^{*}=V_{n}^{*} T$ for this $T$.

Let $J_{n}=I_{n}-\frac{1}{n} l_{n} l_{n}^{\prime}$ and $P_{n}$ be any $n \times n$ matrix with trace zero. The following identities are useful to show the desired results: (a) $\operatorname{vec}_{\mathrm{D}}\left(P_{\alpha n}^{*}\right)=\frac{2}{\left(\eta_{4}-1\right)-\eta_{3}^{2}} \operatorname{vec}_{\mathrm{D}}\left(\mathbb{W}_{n}\right)-\frac{\sigma_{0}^{-1} \eta_{3}}{\left(\eta_{4}-1\right)-\eta_{3}^{2}} J_{n} e^{\tau_{0} M_{n}} W_{n} X_{n} \beta_{0} ;(\mathrm{b}) \operatorname{vec}_{\mathrm{D}}\left(P_{\beta n l}^{*}\right)=$ $J_{n} e^{\tau_{0} M_{n}} X_{n l}^{*}$; (c) $\sum_{l=1}^{k^{*}} \operatorname{vec}_{\mathrm{D}}\left(P_{\beta n l}^{*}\right) e_{k l}^{\prime}=J_{n} e^{\tau_{0} M_{n}} X_{n}$; (d) $\sigma_{0}^{2} F_{\alpha n}^{*}+\mu_{3} \operatorname{vec}_{\mathrm{D}}\left(P_{\alpha n}^{*}\right)=\sigma_{0}^{2} e^{\tau_{0} M_{n}} W_{n} X_{n} \beta_{0}$; (e) $F_{\beta n}^{*}-\frac{\eta_{3}^{2}}{\left(\eta_{4}-1\right)-\eta_{3}^{2}} \sum_{l=1}^{k^{*}} \operatorname{vec}_{\mathrm{D}}\left(P_{\beta n l}^{*}\right) e_{k l}^{\prime}=e^{\tau_{0} M_{n}} X_{n} ;(\mathrm{f}) \operatorname{vec}_{\mathrm{D}}^{\prime}\left(P_{n}\right) F_{\beta n}^{*}=\frac{\eta_{4}-1}{\left(\eta_{4}-1\right)-\eta_{3}^{2}} \operatorname{vec}_{\mathrm{D}}{ }^{\prime}\left(P_{n}\right) e^{\tau_{0} M_{n}} X_{n} ; \quad(\mathrm{g})$ $\mu_{3} \operatorname{vec}_{\mathrm{D}}{ }^{\prime}\left(P_{n}\right) F_{\alpha n}^{*}+\sigma_{0}^{4} \operatorname{tr}\left(P_{n}^{s} P_{\alpha n}^{*}\right)+\left(\mu_{4}-3 \sigma_{0}^{4}\right) \operatorname{vec}_{\mathrm{D}}{ }^{\prime}\left(P_{n}\right) \operatorname{vec}_{\mathrm{D}}\left(P_{\alpha n}^{*}\right)=\sigma_{0}^{4} \operatorname{tr}\left(P_{n}^{s} \mathbb{W}_{n}\right)$.

Since $g_{n}^{*^{\prime}}(\gamma) \Delta_{P F}=\epsilon_{n}^{\prime}(\gamma)\left(P_{\alpha n}^{*} \epsilon_{n}(\gamma), P_{\tau n}^{*} \epsilon_{n}(\gamma), P_{\beta n 1}^{*} \epsilon_{n}(\gamma), \ldots, P_{\beta n k^{*}}^{*} \epsilon_{n}(\gamma), F_{\alpha n}^{*}, F_{\beta n}^{*}\right)$ as shown above and 
$P_{\beta n l}^{*}$ 's are diagonal matrices, we have

$$
\begin{aligned}
& V_{n, 21} \Delta_{P F}=\mathrm{E}\left[g_{n}\left(\gamma_{0}\right)\left(g_{n}^{*^{\prime}}\left(\gamma_{0}\right) \Delta_{P F}\right)\right] \\
& =\frac{1}{n}\left(\begin{array}{cccc}
\sigma_{0}^{4} \operatorname{tr}\left(P_{n 1}^{s} P_{\alpha n}^{*}\right) & \sigma_{0}^{4} \operatorname{tr}\left(P_{n 1}^{s} P_{\tau n}^{*}\right) & \sigma_{0}^{4} \operatorname{vec}_{\mathrm{D}}^{\prime}\left(P_{n 1}^{s}\right)\left(\operatorname{vec}_{\mathrm{D}}\left(P_{\beta n 1}^{*}\right), \ldots, \operatorname{vec}_{\mathrm{D}}\left(P_{\beta n k^{*}}^{*}\right)\right) & \mu_{3} \operatorname{vec}_{\mathrm{D}}^{\prime}\left(P_{n 1}\right)\left(F_{\alpha n}^{*}, F_{\beta n}^{*}\right) \\
\vdots & \vdots & \vdots & \vdots \\
\sigma_{0}^{4} \operatorname{tr}\left(P_{n, k_{p}}^{s} P_{\alpha n}^{*}\right) & \sigma_{0}^{4} \operatorname{tr}\left(P_{n, k_{p}}^{s} P_{\tau n}^{*}\right) & \sigma_{0}^{4} \operatorname{vec}_{\mathrm{D}}^{\prime}\left(P_{n, k_{p}}^{s}\right)\left(\operatorname{vec}_{\mathrm{D}}\left(P_{\beta n 1}^{*}\right), \ldots, \operatorname{vec}_{\mathrm{D}}\left(P_{\beta n k^{*}}^{*}\right)\right) & \mu_{3} \operatorname{vec}_{\mathrm{D}}^{\prime}\left(P_{n, k_{p}}\right)\left(F_{\alpha n}^{*}, F_{\beta n}^{*}\right) \\
\mu_{3} F_{n}^{\prime} \operatorname{vec}_{\mathrm{D}}\left(P_{\alpha n}^{*}\right) & \mu_{3} F_{n}^{\prime} \operatorname{vec}_{\mathrm{D}}\left(P_{\tau n}^{*}\right) & \mu_{3} F_{n}^{\prime}\left(\operatorname{vec}_{\mathrm{D}}\left(P_{\beta n 1}^{*}\right), \ldots, \operatorname{vec}_{\mathrm{D}}\left(P_{\beta n k^{*}}^{*}\right)\right) & \sigma_{0}^{2} F_{n}^{\prime}\left(F_{\alpha n}^{*}, F_{\beta n}^{*}\right)
\end{array}\right) \\
& +\frac{\mu_{4}-3 \sigma_{0}^{4}}{n}\left(\begin{array}{cccc}
\operatorname{vec}_{\mathrm{D}}^{\prime}\left(P_{n 1}\right) \operatorname{vec}_{\mathrm{D}}\left(P_{\alpha n}^{*}\right) & \operatorname{vec}_{\mathrm{D}}{ }^{\prime}\left(P_{n 1}\right) \operatorname{vec}_{\mathrm{D}}\left(P_{\tau n}^{*}\right) & \operatorname{vec}_{\mathrm{D}}^{\prime}\left(P_{n 1}\right)\left(\operatorname{vec}_{\mathrm{D}}\left(P_{\beta n 1}^{*}\right), \ldots, \operatorname{vec}_{\mathrm{D}}\left(P_{\beta n k^{*}}^{*}\right)\right) & 0 \\
\vdots & \vdots & \vdots & \vdots \\
\operatorname{vec}_{\mathrm{D}}^{\prime}\left(P_{n, k_{p}}\right) \operatorname{vec}_{\mathrm{D}}\left(P_{\alpha n}^{*}\right) & \operatorname{vec}_{\mathrm{D}}{ }^{\prime}\left(P_{n, k_{p}}\right) \operatorname{vec}_{\mathrm{D}}\left(P_{\tau n}^{*}\right) & \operatorname{vec}_{\mathrm{D}}{ }^{\prime}\left(P_{n, k_{p}}\right)\left(\operatorname{vec}_{\mathrm{D}}\left(P_{\beta n 1}^{*}\right), \ldots, \operatorname{vec}_{\mathrm{D}}\left(P_{\beta n k^{*}}^{*}\right)\right) & 0 \\
0 & \ldots & 0 & 0
\end{array}\right) .
\end{aligned}
$$

The $V_{n, 21} T=\left(V_{n, 21} \Delta_{P F}\right) \Delta_{T}$ is a $\left(k_{p}+1\right) \times 3$ block matrix. By $(\mathrm{g})$, the $(j, 1)$ th block of $V_{n, 21} T$ for $1 \leq j \leq k_{p}$ is $\frac{1}{n} \sigma_{0}^{2} \operatorname{tr}\left(P_{n j}^{s} \mathbb{W}_{n}\right)$; the $(j, 2)$ th block of $V_{n, 21} T$ for $1 \leq j \leq k_{p}$ is $\frac{1}{n} \sigma_{0}^{2} \operatorname{tr}\left(P_{n j}^{s} M_{n}\right)$; by (c) and (f), the $(j, 3) \operatorname{th}$ block of $V_{n, 21} T$ for $1 \leq j \leq k_{p}$ is 0 ; by (d), the $\left(k_{p}+1,1\right)$ th block of $V_{n, 21} T$ is $\frac{1}{n} F_{n}^{\prime} e^{\tau_{0} M_{n}} W_{n} X_{n} \beta_{0}$; the $\left(k_{p}+1,2\right)$ th block of $V_{n, 21} T$ is 0 ; by $(\mathrm{e})$, the $\left(k_{p}+1,3\right)$ th block of $V_{n, 21} T$ is $-\frac{1}{n} F_{n}^{\prime} e^{\tau_{0} M_{n}} X_{n}$. Thus $V_{n, 21} T=G_{n}$.

Furthermore, as $g_{n}^{*}(\gamma)$ is a special case of $g_{n}(\gamma), G_{n}^{*}=V_{n}^{*} T$. Then $\Lambda_{n}^{*}=G_{n}^{*^{\prime}} V_{n}^{*-1} G_{n}^{*}=G_{n}^{*^{\prime}} T=$ $\left(G_{n}^{*^{\prime}} \Delta_{P F}\right) \Delta_{T}=\mathrm{E} \frac{\partial\left(g_{n}^{*^{\prime}}\left(\gamma_{0}\right) \Delta_{P F}\right)}{\partial \gamma_{0}} \Delta_{T}$, which has the explicit expression in (30) by some computation. The asymptotic distribution of $\hat{\gamma}_{n}^{*}$ follows by Proposition 6 .

Proof of Proposition A.1. The consistency of the QMLE $\hat{\gamma}_{n}$ will follow from the uniform convergence that $\sup _{\boldsymbol{\phi} \in \boldsymbol{\Phi}} \frac{1}{n}\left|Q_{n}(\phi)-\bar{Q}_{n}(\phi)\right|=o_{P}(1)$ and the identification uniqueness condition.

We first show the uniform convergence. As $y_{n}=e^{-\boldsymbol{\alpha}_{0} \mathbf{W}_{n}}\left(X_{n} \beta_{0}+e^{-\boldsymbol{\tau}_{0} \mathbf{M}_{n}} \epsilon_{n}\right)$,

$$
\begin{aligned}
\frac{1}{n}\left[Q_{n}(\boldsymbol{\phi})-\bar{Q}_{n}(\boldsymbol{\phi})\right]= & \frac{2}{n}\left(X_{n} \beta_{0}\right)^{\prime} e^{-\left(\boldsymbol{\alpha}_{0} \mathbf{W}_{n}\right)^{\prime}} e^{\left(\boldsymbol{\alpha} \mathbf{W}_{n}\right)^{\prime}} e^{\left(\boldsymbol{\tau} \mathbf{M}_{n}\right)^{\prime}} H_{n}(\boldsymbol{\tau}) e^{\boldsymbol{\tau} \mathbf{M}_{n}} e^{\boldsymbol{\alpha} \mathbf{W}_{n}} e^{-\boldsymbol{\alpha}_{0} \mathbf{W}_{n}} e^{-\boldsymbol{\tau}_{0} \mathbf{M}_{n}} \epsilon_{n} \\
& +\frac{1}{n} \epsilon_{n}^{\prime} e^{-\left(\boldsymbol{\tau}_{0} \mathbf{M}_{n}\right)^{\prime}} e^{-\left(\boldsymbol{\alpha}_{0} \mathbf{W}_{n}\right)^{\prime}} e^{\left(\boldsymbol{\alpha} \mathbf{W}_{n}\right)^{\prime}} e^{\left(\boldsymbol{\tau} \mathbf{M}_{n}\right)^{\prime}} H_{n}(\boldsymbol{\tau}) e^{\boldsymbol{\tau} \mathbf{M}_{n}} e^{\boldsymbol{\alpha} \mathbf{W}_{n}} e^{-\boldsymbol{\alpha}_{0} \mathbf{W}_{n}} e^{-\boldsymbol{\tau}_{0} \mathbf{M}_{n}} \epsilon_{n} \\
& -\frac{\sigma_{0}^{2}}{n} \operatorname{tr}\left[e^{-\left(\boldsymbol{\tau}_{0} \mathbf{M}_{n}\right)^{\prime}} e^{-\left(\boldsymbol{\alpha}_{0} \mathbf{W}_{n}\right)^{\prime}} e^{\left(\boldsymbol{\alpha} \mathbf{W}_{n}\right)^{\prime}} e^{\left(\boldsymbol{\tau} \mathbf{M}_{n}\right)^{\prime}} H_{n}(\boldsymbol{\tau}) e^{\boldsymbol{\tau} \mathbf{M}_{n}} e^{\boldsymbol{\alpha} \mathbf{W}_{n}} e^{-\boldsymbol{\alpha}_{0} \mathbf{W}_{n}} e^{-\boldsymbol{\tau}_{0} \mathbf{M}_{n}}\right] \\
& -\frac{\sigma_{0}^{2}}{n} \operatorname{tr}\left\{e^{-\left(\boldsymbol{\tau}_{0} \mathbf{M}_{n}\right)^{\prime}} e^{-\left(\boldsymbol{\alpha}_{0} \mathbf{W}_{n}\right)^{\prime}} e^{\left(\boldsymbol{\alpha} \mathbf{W}_{n}\right)^{\prime}} e^{\left(\boldsymbol{\tau} \mathbf{M}_{n}\right)^{\prime}}\left[I_{n}-H_{n}(\boldsymbol{\tau})\right] e^{\boldsymbol{\tau} \mathbf{M}_{n}} e^{\boldsymbol{\alpha} \mathbf{W}_{n}} e^{-\boldsymbol{\alpha}_{0} \mathbf{W}_{n}} e^{-\boldsymbol{\tau}_{0} \mathbf{M}_{n}}\right\}
\end{aligned}
$$

By Lemma 10, $\frac{1}{n}\left[Q_{n}(\phi)-\bar{Q}_{n}(\phi)\right]=o_{P}(1)$ uniformly on $\boldsymbol{\Phi}$. 
We now show that $\frac{1}{n} \bar{Q}_{n}(\phi)$ is uniformly equicontinuous. By the mean value theorem, for $\phi, \phi^{*} \in \mathbf{\Phi}$,

$$
\begin{aligned}
& \frac{1}{n}\left[\bar{Q}_{n}\left(\boldsymbol{\phi}^{*}\right)-\bar{Q}_{n}(\boldsymbol{\phi})\right] \\
& =2 \sum_{i=1}^{p}\left(X_{n} \beta_{0}\right)^{\prime} e^{-\left(\boldsymbol{\alpha}_{0} \mathbf{W}_{n}\right)^{\prime}} e^{\left(\tilde{\boldsymbol{\alpha}} \mathbf{W}_{n}\right)^{\prime}} e^{\left(\tilde{\boldsymbol{\tau}} \mathbf{M}_{n}\right)^{\prime}} H_{n}(\tilde{\boldsymbol{\tau}}) e^{\tilde{\boldsymbol{\tau}} \mathbf{M}_{n}} \frac{\partial e^{\tilde{\boldsymbol{\alpha}} \mathbf{W}_{n}}}{\partial \alpha_{i}} e^{-\boldsymbol{\alpha}_{0} \mathbf{W}_{n}} X_{n} \beta_{0}\left(\alpha_{i}^{*}-\alpha_{i}\right) \\
& \quad+\frac{2 \sigma_{0}^{2}}{n} \sum_{i=1}^{p} \operatorname{tr}\left[e^{-\left(\boldsymbol{\tau}_{0} \mathbf{M}_{n}\right)^{\prime}} e^{-\left(\boldsymbol{\alpha}_{0} \mathbf{W}_{n}\right)^{\prime}} e^{\left(\tilde{\boldsymbol{\alpha}} \mathbf{W}_{n}\right)^{\prime}} e^{\left(\tilde{\boldsymbol{\tau}} \mathbf{M}_{n}\right)^{\prime}} e^{\tilde{\boldsymbol{\tau}} \mathbf{M}_{n}} \frac{\partial e^{\tilde{\boldsymbol{\alpha}} \mathbf{W}_{n}}}{\partial \alpha_{i}} e^{-\boldsymbol{\alpha}_{0} \mathbf{W}_{n}} e^{-\boldsymbol{\tau}_{0} \mathbf{M}_{n}}\right]\left(\alpha_{i}^{*}-\alpha_{i}\right) \\
& +\frac{1}{n} \sum_{i=1}^{q}\left(X_{n} \beta_{0}\right)^{\prime} e^{-\left(\boldsymbol{\alpha}_{0} \mathbf{W}_{n}\right)^{\prime}} e^{\left(\tilde{\boldsymbol{\alpha}} \mathbf{W}_{n}\right)^{\prime}} e^{\left(\tilde{\boldsymbol{\tau}} \mathbf{M}_{n}\right)^{\prime}}\left(2 H_{n}(\tilde{\boldsymbol{\tau}}) \frac{\partial e^{\tilde{\boldsymbol{\tau}} \mathbf{M}_{n}}}{\partial \tau_{i}}+\frac{\partial H_{n}(\tilde{\boldsymbol{\tau}})}{\partial \tau_{i}} e^{\left.\tilde{\boldsymbol{\tau}} \mathbf{M}_{n}\right)} e^{\tilde{\boldsymbol{\alpha}} \mathbf{W}_{n}} e^{-\boldsymbol{\alpha}_{0} \mathbf{W}_{n}} X_{n} \beta_{0}\left(\tau_{i}^{*}-\tau_{i}\right)\right. \\
& \quad+\frac{2 \sigma_{0}^{2}}{n} \sum_{i=1}^{q} \operatorname{tr}\left[e^{-\left(\boldsymbol{\tau}_{0} \mathbf{M}_{n}\right)^{\prime}} e^{-\left(\boldsymbol{\alpha}_{0} \mathbf{W}_{n}\right)^{\prime}} e^{\left(\tilde{\boldsymbol{\alpha}} \mathbf{W}_{n}\right)^{\prime}} e^{\left(\tilde{\boldsymbol{\tau}} \mathbf{M}_{n}\right)^{\prime}} \frac{\partial e^{\tilde{\boldsymbol{\tau}} \mathbf{M}_{n}}}{\partial \tau_{i}} e^{\tilde{\boldsymbol{\alpha}} \mathbf{W}_{n}} e^{-\boldsymbol{\alpha}_{0} \mathbf{W}_{n}} e^{-\boldsymbol{\tau}_{0} \mathbf{M}_{n}}\right]\left(\tau_{i}^{*}-\tau_{i}\right)
\end{aligned}
$$

where $\tilde{\boldsymbol{\phi}}$ is between $\boldsymbol{\phi}^{*}$ and $\boldsymbol{\phi}$. By Lemma $9, H_{n}(\boldsymbol{\tau}), e^{\boldsymbol{\alpha} \mathbf{W}_{n}}$ and $e^{\boldsymbol{\tau} \mathbf{M}_{n}}$ are UB uniformly over their respective parameter spaces. By the proof of Lemma 10, $\frac{\partial e^{\alpha \mathbf{W}_{n}}}{\partial \alpha_{i}}, \frac{\partial e^{\boldsymbol{\tau} \mathbf{M}_{n}}}{\partial \tau_{i}}$ and $\frac{\partial H_{n}(\boldsymbol{\tau})}{\partial \tau_{i}}$ are UB uniformly over their respective parameter spaces. Then there exists some constant $c$ such that

$$
\frac{1}{n}\left|\bar{Q}_{n}\left(\phi^{*}\right)-\bar{Q}_{n}(\boldsymbol{\phi})\right| \leq c\left(\left\|\boldsymbol{\alpha}^{*}-\boldsymbol{\alpha}\right\|+\left\|\boldsymbol{\tau}^{*}-\boldsymbol{\tau}\right\|\right) .
$$

Thus $\frac{1}{n} \bar{Q}_{n}(\phi)$ is uniformly equicontinuous.

Finally, we show that the identification uniqueness condition holds. Let $\lambda_{1}, \ldots, \lambda_{n}$ be the eigenvalues of $A_{n}(\boldsymbol{\phi})=e^{-\left(\boldsymbol{\tau}_{0} \mathbf{M}_{n}\right)^{\prime}} e^{-\left(\boldsymbol{\alpha}_{0} \mathbf{W}_{n}\right)^{\prime}} e^{\left(\boldsymbol{\alpha} \mathbf{W}_{n}\right)^{\prime}} e^{\left(\boldsymbol{\tau} \mathbf{M}_{n}\right)^{\prime}} e^{\boldsymbol{\tau} \mathbf{M}_{n}} e^{\boldsymbol{\alpha} \mathbf{W}_{n}} e^{-\boldsymbol{\alpha}_{0} \mathbf{W}_{n}} e^{-\boldsymbol{\tau}_{0} \mathbf{M}_{n}}$. Since $A_{n}(\boldsymbol{\phi})$ is positive definite, $\lambda_{i}$ 's are all positive. Then by the inequality of arithmetic and geometric means,

$$
\begin{aligned}
\frac{1}{n} \operatorname{tr}\left(A_{n}(\boldsymbol{\phi})\right)=\frac{1}{n} \sum_{i=1}^{n} \lambda_{i} & \geq\left(\prod_{i=1}^{n} \lambda_{i}\right)^{1 / n}=\left|A_{n}(\boldsymbol{\phi})\right|^{1 / n} \\
& =\left[e^{-\operatorname{tr}\left(\boldsymbol{\tau}_{0} \mathbf{M}_{n}\right)} e^{-\operatorname{tr}\left(\boldsymbol{\alpha}_{0} \mathbf{W}_{n}\right)} e^{\operatorname{tr}\left(\boldsymbol{\alpha} \mathbf{W}_{n}\right)} e^{\operatorname{tr}\left(\boldsymbol{\tau} \mathbf{M}_{n}\right)} e^{\operatorname{tr}\left(\boldsymbol{\tau} \mathbf{M}_{n}\right)} e^{\operatorname{tr}\left(\boldsymbol{\alpha} \mathbf{W}_{n}\right)} e^{-\operatorname{tr}\left(\boldsymbol{\alpha}_{0} \mathbf{W}_{n}\right)} e^{-\operatorname{tr}\left(\boldsymbol{\tau}_{0} \mathbf{M}_{n}\right)}\right]^{1 / n} \\
& =1,
\end{aligned}
$$

because $\operatorname{tr}\left(\boldsymbol{\alpha} \mathbf{M}_{n}\right)=\operatorname{tr}\left(\boldsymbol{\tau} \mathbf{W}_{n}\right)=0$. In addition,

$$
\left(X_{n} \beta_{0}\right)^{\prime} e^{-\left(\boldsymbol{\alpha}_{0} \mathbf{W}_{n}\right)^{\prime}} e^{\left(\boldsymbol{\alpha} \mathbf{W}_{n}\right)^{\prime}} e^{\left(\boldsymbol{\tau} \mathbf{M}_{n}\right)^{\prime}} H_{n}(\boldsymbol{\tau}) e^{\boldsymbol{\tau} \mathbf{M}_{n}} e^{\boldsymbol{\alpha} \mathbf{W}_{n}} e^{-\boldsymbol{\alpha}_{0} \mathbf{W}_{n}} X_{n} \beta_{0} \geq 0
$$

Thus, $\frac{1}{n} \bar{Q}_{n}(\phi) \geq \sigma_{0}^{2}$. When $\phi=\phi_{0}, \frac{1}{n} \bar{Q}_{n}(\phi)=\sigma_{0}^{2}$. Assumption 6 implies that whenever $\phi \neq \phi_{0}$, $\lim _{n \rightarrow \infty} \frac{1}{n} \bar{Q}_{n}(\phi) \neq \sigma_{0}^{2}$. Thus the identification uniqueness condition holds. 
With the uniform convergence and identification uniqueness condition, the consistency of $\hat{\phi}_{n}$ follows. The consistency of $\hat{\beta}_{n}$ follows by plugging $\hat{\phi}_{n}$ into the function $\hat{\beta}_{n}(\phi)$ in (A.4).

Proof of Proposition A.2. To obtain the asymptotic distribution, we use (A.7) for $\sqrt{n}\left(\hat{\gamma}_{n}-\gamma_{0}\right)$ derived from a Taylor expansion of the first-order condition. We first show that $\frac{1}{n} \frac{\partial^{2} Q_{n}\left(\tilde{\gamma}_{n}\right)}{\partial \gamma \partial \gamma^{\prime}}=\frac{1}{n} \frac{\partial^{2} Q_{n}\left(\gamma_{0}\right)}{\partial \gamma \partial \gamma^{\prime}}+o_{P}(1)=$ $\frac{1}{n} \mathrm{E} \frac{\partial^{2} Q_{n}\left(\boldsymbol{\gamma}_{0}\right)}{\partial \gamma \partial \gamma^{\prime}}+o_{P}(1)=\mathbf{C}_{n}+o_{P}(1)$. Noting that for any $\boldsymbol{\alpha}^{*}, \boldsymbol{\alpha} \in[-\delta, \delta]^{p}$, by expanding $\left(\boldsymbol{\alpha}^{*} \mathbf{W}_{n}\right)^{i}=$ $\left[\left(\boldsymbol{\alpha}^{*}-\boldsymbol{\alpha}\right) \mathbf{W}_{n}+\boldsymbol{\alpha} \mathbf{W}_{n}\right]^{i}$, we have

$$
\begin{aligned}
\left\|\left(\boldsymbol{\alpha}^{*} \mathbf{W}_{n}\right)^{i}-\left(\boldsymbol{\alpha} \mathbf{W}_{n}\right)^{i}\right\|_{\infty} & \leq \sum_{k=1}^{i}\left(\begin{array}{c}
i \\
k
\end{array}\right)\left\|\boldsymbol{\alpha} \mathbf{W}_{n}\right\|_{\infty}^{i-k}\left\|\left(\boldsymbol{\alpha}^{*}-\boldsymbol{\alpha}\right) \mathbf{W}_{n}\right\|_{\infty}^{k} \\
& =i\left\|\left(\boldsymbol{\alpha}^{*}-\boldsymbol{\alpha}\right) \mathbf{W}_{n}\right\|_{\infty} \sum_{k=1}^{i} \frac{1}{k}\left(\begin{array}{c}
i-1 \\
k-1
\end{array}\right)\left\|\boldsymbol{\alpha} \mathbf{W}_{n}\right\|_{\infty}^{i-k}\left\|\left(\boldsymbol{\alpha}^{*}-\boldsymbol{\alpha}\right) \mathbf{W}_{n}\right\|_{\infty}^{k-1} \\
& \leq i\left\|\left(\boldsymbol{\alpha}^{*}-\boldsymbol{\alpha}\right) \mathbf{W}_{n}\right\|_{\infty}\left(\left\|\boldsymbol{\alpha} \mathbf{W}_{n}\right\|_{\infty}+\left\|\left(\boldsymbol{\alpha}^{*}-\boldsymbol{\alpha}\right) \mathbf{W}_{n}\right\|_{\infty}\right)^{i-1}
\end{aligned}
$$

Then $\left\|e^{\boldsymbol{\alpha}^{*} \mathbf{W}_{n}}-e^{\boldsymbol{\alpha} \mathbf{W}_{n}}\right\|_{\infty} \leq\left\|\left(\boldsymbol{\alpha}^{*}-\boldsymbol{\alpha}\right) \mathbf{W}_{n}\right\|_{\infty}\left\|\sum_{i=1}^{\infty} \frac{1}{(i-1) !}\left(\left\|\boldsymbol{\alpha} \mathbf{W}_{n}\right\|_{\infty}+\left\|\left(\boldsymbol{\alpha}^{*}-\boldsymbol{\alpha}\right) \mathbf{W}_{n}\right\|_{\infty}\right)^{i-1}=\right\|\left(\boldsymbol{\alpha}^{*}-\right.$ $\boldsymbol{\alpha}) \mathbf{W}_{n}\left\|_{\infty} e^{\left\|\boldsymbol{\alpha} \mathbf{W}_{n}\right\|_{\infty}+\left\|\left(\boldsymbol{\alpha}^{*}-\boldsymbol{\alpha}\right) \mathbf{W}_{n}\right\|_{\infty}} \leq\right\| \boldsymbol{\alpha}^{*}-\boldsymbol{\alpha}\left\|\max _{1 \leq j \leq p}\right\| W_{n j} \|_{\infty} e^{\left\|\boldsymbol{\alpha} \mathbf{W}_{n}\right\|_{\infty}+\left\|\left(\boldsymbol{\alpha}^{*}-\boldsymbol{\alpha}\right) \mathbf{W}_{n}\right\|_{\infty}}$. Thus $\| e^{\tilde{\boldsymbol{\alpha}}_{n} \mathbf{W}_{n}-}$ $e^{\boldsymbol{\alpha}_{0} \mathbf{W}_{n}} \|_{\infty}=o_{P}(1)$. With this result, we can show that $\frac{1}{n} \frac{\partial^{2} Q_{n}\left(\tilde{\boldsymbol{\gamma}}_{n}\right)}{\partial \gamma \partial \gamma^{\prime}}=\frac{1}{n} \frac{\partial^{2} Q_{n}\left(\gamma_{0}\right)}{\partial \gamma \partial \gamma^{\prime}}+o_{P}(1) .{ }^{35}$ Furthermore, each element of $\frac{1}{n} \frac{\partial^{2} Q_{n}\left(\gamma_{0}\right)}{\partial \gamma \partial \gamma^{\prime}}-\frac{1}{n} \mathrm{E} \frac{\partial^{2} Q_{n}\left(\gamma_{0}\right)}{\partial \gamma \partial \gamma^{\prime}}$ is a linear-quadratic function of $\epsilon_{n}$, then $\frac{1}{n} \frac{\partial^{2} Q_{n}\left(\gamma_{0}\right)}{\partial \gamma \partial \gamma^{\prime}}=$ $\frac{1}{n} \mathrm{E} \frac{\partial^{2} Q_{n}\left(\gamma_{0}\right)}{\partial \gamma \partial \gamma^{\prime}}+o_{P}(1)$ by Lemma 4. Hence, $\frac{1}{n} \frac{\partial^{2} Q_{n}\left(\tilde{\boldsymbol{\gamma}}_{n}\right)}{\partial \gamma \partial \gamma^{\prime}}=\frac{1}{n} \mathrm{E} \frac{\partial^{2} Q_{n}\left(\gamma_{0}\right)}{\partial \gamma \partial \gamma^{\prime}}+o_{P}(1)$. It follows that

$$
\sqrt{n}\left(\hat{\gamma}_{n}-\gamma_{0}\right)=-\mathbf{C}_{n}^{-1} \frac{1}{\sqrt{n}} \frac{\partial Q_{n}\left(\gamma_{0}\right)}{\partial \gamma}+o_{P}(1)
$$

where each element of $\frac{\partial Q_{n}\left(\gamma_{0}\right)}{\partial \gamma}$ is a linear-quadratic form of $\epsilon_{n}$ as shown in (A.8)-(A.10). We now show that $\mathrm{E} \frac{\partial Q_{n}\left(\boldsymbol{\gamma}_{0}\right)}{\partial \gamma}=0$. As $\epsilon_{n i}$ 's are i.i.d. with mean zero, it remains to show that $\operatorname{tr}\left(e^{-\left(\boldsymbol{\tau}_{0} \mathbf{M}_{n}\right)^{\prime}} e^{-\left(\boldsymbol{\alpha}_{0} \mathbf{W}_{n}\right)^{\prime}} \frac{\partial e^{\left(\boldsymbol{\alpha}_{0} \mathbf{W}_{n}\right)^{\prime}}}{\partial \alpha_{i}} e^{\left(\boldsymbol{\tau}_{0} \mathbf{M}_{n}\right)^{\prime}}\right)=$ $\operatorname{tr}\left(\frac{\partial e^{\boldsymbol{\alpha}_{0} \mathbf{W}_{n}}}{\partial \alpha_{i}} e^{-\boldsymbol{\alpha}_{0} \mathbf{W}_{n}}\right)=0$ and $\operatorname{tr}\left(\frac{\partial e^{\tau_{0} \mathbf{M}_{n}}}{\partial \tau_{i}} e^{-\tau_{0} \mathbf{M}_{n}}\right)=0$. W.l.o.g., we show that $\operatorname{tr}\left(\frac{\partial e^{\boldsymbol{\alpha}_{0} \mathbf{W}_{n}}}{\partial \alpha_{i}} e^{-\boldsymbol{\alpha}_{0} \mathbf{W}_{n}}\right)=0$. As $\frac{\partial e^{\boldsymbol{\alpha}_{0} \mathbf{W}_{n}}}{\partial \alpha_{i}}=\sum_{j=1}^{\infty} \sum_{k=0}^{j-1} \frac{1}{j !}\left(\boldsymbol{\alpha}_{0} \mathbf{W}_{n}\right)^{k} W_{n i}\left(\boldsymbol{\alpha}_{0} \mathbf{W}_{n}\right)^{j-1-k},\left(\boldsymbol{\alpha}_{0} \mathbf{W}_{n}\right)^{j} e^{-\boldsymbol{\alpha}_{0} \mathbf{W}_{n}}=e^{-\boldsymbol{\alpha}_{0} \mathbf{W}_{n}}\left(\boldsymbol{\alpha}_{0} \mathbf{W}_{n}\right)^{j}$ and $\operatorname{tr}(A B)=$ $\operatorname{tr}(B A)$ for any two conformable square matrices $A$ and $B$, we have

$$
\begin{aligned}
\operatorname{tr}\left(\frac{\partial e^{\boldsymbol{\alpha}_{0} \mathbf{W}_{n}}}{\partial \alpha_{i}} e^{-\boldsymbol{\alpha}_{0} \mathbf{W}_{n}}\right) & =\operatorname{tr}\left(W_{n i} e^{-\boldsymbol{\alpha}_{0} \mathbf{W}_{n}} \sum_{j=1}^{\infty} \frac{1}{j !} \sum_{k=0}^{j-1}\left(\boldsymbol{\alpha}_{0} \mathbf{W}_{n}\right)^{j-1}\right) \\
& =\operatorname{tr}\left(W_{n i} e^{-\boldsymbol{\alpha}_{0} \mathbf{W}_{n}} \sum_{j=1}^{\infty} \frac{1}{(j-1) !}\left(\boldsymbol{\alpha}_{0} \mathbf{W}_{n}\right)^{j-1}\right) \\
& =\operatorname{tr}\left(W_{n i}\right)=0 .
\end{aligned}
$$

\footnotetext{
${ }^{35}$ Please see the proof of Proposition 2 in the supplement for a similar argument.
} 
Applying the central limit theorem in Lemma 5, we have

$$
\sqrt{n}\left(\hat{\gamma}_{n}-\gamma_{0}\right) \stackrel{d}{\rightarrow} N\left(0, \lim _{n \rightarrow \infty} \mathbf{C}_{n}^{-1} \mathbf{\Omega}_{n} \mathbf{C}_{n}^{-1}\right)
$$

where $\boldsymbol{\Omega}_{n}=\frac{1}{n} \mathrm{E}\left(\frac{\partial Q_{n}\left(\boldsymbol{\gamma}_{0}\right)}{\partial \boldsymbol{\gamma}} \frac{\partial Q_{n}\left(\boldsymbol{\gamma}_{0}\right)}{\partial \boldsymbol{\gamma}^{\prime}}\right)$. The explicit expressions for $\mathbf{C}_{n}$ and $\boldsymbol{\Omega}_{n}$ can be derived by Lemma 3 .

\section{Acknowledgments}

We would like to thank Yvan Stroppa and Matthias Pécot for providing valuable help with the Monte Carlo simulations and seminar participants of the economics department of the University of Murcia and University of Orléans.

\section{References}

Amemiya, T. (1985), Advanced econometrics, Harvard University Press, Cambridge, MA.

Baltagi, B. H., Egger, P. and Pfaffermayr, M. (2007), 'Estimating models of complex FDI: Are there third-country effects', Journal of Econometrics 140, 260-281.

Baltagi, B. H., Egger, P. and Pfaffermayr, M. (2008), 'Estimating regional trade agreement effects on FDI in an interdependent world', Journal of Econometrics 145, 194-208.

Blonigen, B. A., Davies, R. B., Waddell, G. R. and Naughton, H. T. (2007), 'FDI in space: Spatial autoregressive relationships in foreign direct investment', European Economic review 51, 1303-1325.

Breusch, T., Qian, H., Schmidt, P. and Wyhowski, D. (1999), 'Redundancy of moment conditions', Journal of Econometrics 91, 89-111.

Chiu, T. Y. M., Leonard, T. and Tsui, K.-W. (1996), 'The matrix-logarithmic covariance model', Journal of the American Statistical Association 91(433), 198-210.

Coughlin, C. C. and Segev, E. (2000), 'Foreign direct investment in China: A spatial econometric study', The World Economy 23, $1-23$.

Davies, R. B. (2005), 'Fragmentation of headquarter services and FDI', North American Journal of Economics and Finance 16, 61-79.

Debarsy, N. and Ertur, C. (2010), 'Testing for spatial autocorrelation in a fixed effects panel data model', Regional Science and Urban Economics 40, 453-470.

Elhorst, J. P., Lacombe, D. J. and Piras, G. (2012), 'On model specification and parameter space definitions in higher order spatial econometric models', Regional Science and Urban Economics 42(1), 211-220.

Garretsen, H. and Peeters, J. (2009), 'FDI and the relevance of spatial linkages: do third country effects matter for Dutch FDI?', Review of World Economics 145, 319-338.

Jin, F. and Lee, L.-F. (2012), 'Approximated likelihood and root estimators for spatial interaction in spatial autoregressive models', Regional Science and Urban Economics 42, 446-458. 
Kelejian, H. H. and Prucha, I. R. (1998), 'A generalized spatial two-stage least squares procedure for estimating a spatial autoregressive model with autoregressive disturbances', Journal of Real Estate Finance and Economics 17, 99-121.

Kelejian, H. H. and Prucha, I. R. (2001), 'On the asymptotic distribution of the Moran I test statistic with applications', Journal of Econometrics 104, 219-257.

Kelejian, H. H. and Prucha, I. R. (2010), 'Specification and estimation of spatial autoregressive models with autoregressive and heteroskedastic disturbances', Journal of Econometrics 157, 53-67.

Lee, L.-F. (2003), 'Best spatial two-stage least squares estimators for a spatial autoregressive model with autoregressive disturbances', Econometric Reviews 22(4), 307-335.

Lee, L.-F. (2004), 'Asymptotic distributions of quasi-maximum likelihood estimators for spatial autoregressive models', Econometrica 72(6), 1899-1925.

Lee, L.-F. (2007), 'GMM and 2SLS estimation of mixed regressive, spatial autoregressive models', Journal of Econometrics 137, 489-514.

Lee, L.-F. and Liu, X. (2010), 'Efficient GMM estimation of high order spatial autoregressive models with autoregressive disturbances', Econometric Theory 26, 187-230.

LeSage, J. P. and Pace, R. K. (2007), 'A matrix exponential spatial specification', Journal of Econometrics 140, $190-214$.

LeSage, J. and Pace, R. K. (2009), Introduction to spatial econometrics, Vol. 196, Chapman \& Hall/CRC.

Lin, X. and Lee, L.-F. (2010), 'GMM estimation of spatial autoregressive models with unknown heteroskedasticity', Journal of Econometrics 157, 34-52.

Liu, X., Lee, L.-F. and Bollinger, C. R. (2010), 'An efficient GMM estimator of spatial autoregressive models', Journal of Econometrics 159, 303-319.

Pace, K. R., LeSage, J. P. and Zhu, S. (2011), Spatial dependence in regressors and its effect on estimator performance, Technical report.

Smith, T. E. (2009), 'Estimation bias in spatial models with strongly connected weight matrices', Geographical Analysis 41, 307-332.

White, H. (1980), 'A heteroskedasticity-consistent covariance matrix estimator and a direct test for heteroskedasticity', Econometrica 48, 817-838. 